\title{
CLUSTERING AND THE HYPERBOLIC GEOMETRY OF COMPLEX NETWORKS
}

\author{
Elisabetta Candellero ${ }^{1}$ and Nikolaos Fountoulakis ${ }^{2}$ \\ ${ }^{1}$ Department of Statistics, University of Warwick, Coventry, United Kingdom \\ ${ }^{2}$ School of Mathematics, University of Birmingham, Edgbaston, United Kingdom
}

\begin{abstract}
Clustering is a fundamental property of complex networks and it is the mathematical expression of a ubiquitous phenomenon that arises in various types of self-organized networks such as biological networks, computer networks, or social networks. In this article, we consider what is called the global clustering coefficient of random graphs on the hyperbolic plane. This model of random graphs was proposed recently by Krioukov and colleagues as a mathematical model of complex networks, under the fundamental assumption that hyperbolic geometry underlies the structure of these networks. We give a rigorous analysis of clustering and characterize the global clustering coefficient in terms of the parameters of the model. We show how the global clustering coefficient can be tuned by these parameters and we give an explicit formula for this function.
\end{abstract}

\section{INTRODUCTION}

The theory of complex networks was developed during the last 15 years mainly as a unifying mathematical framework for modeling a variety of networks such as biological networks or large computer networks among which are the Internet, the World Wide Web, as well as social networks that have been recently developed over these platforms. A number of mathematical models have emerged whose aim is to describe fundamental characteristics of these networks as they have been described by experimental evidence; see, for example, [1]. Loosely speaking, the notion of a complex network refers to a class of large networks that exhibit the following characteristics:

1. They are sparse, that is, the number of their edges is proportional to the number of nodes.

2. They exhibit the small world phenomenon: most pairs of vertices that belong to the same component are within a short distance of each other.

3. Clustering: two nodes of the network that have a common neighbour are somewhat more likely to be connected to each other.

An extended abstract of this paper appeared in the Proceedings of the 11th Workshop on Algorithms and Models for the Web Graph (WAW '14), Beijing, China, December 2014.

Address correspondence to Nikolaos Fountoulakis, School of Mathematics, University of Birmingham, Edgbaston B15 2TT, United Kingdom. Email: n.fountoulakis@bham.ac.uk 
4. The tail of their degree distribution follows a power law. In particular, experimental evidence (see [1]) indicates that many networks that emerge in applications follow power-law degree distribution with exponent between 2 and 3 .

The books of [12] and [14] are excellent references for a detailed discussion of these properties.

Among the most influential models was the Watts-Strogatz model of small worlds [30] and the Barabási-Albert model [3], which is also known as the preferential attachment model. The main typical characteristics of these networks have to do with the distribution of the degrees (e.g., power-law distribution), the existence of clustering as well as the typical distances between vertices (e.g., the small-world effect). These models as well as other known models, such as the Chung-Lu model [10, 11], fail to capture all the above features simultaneously or if they do so, they do it in a way that makes it difficult to tune these features independently. For example, the Barabási-Albert model exhibits a power-law degree distribution, with exponent between 2 and 3, and average distance of $\operatorname{order} O(\log \log N)$, when it is suitably parameterized. However, it is locally tree like around a typical vertex (cf. $[7,15])$. On the other hand, theWatts-Strogatz model, although it exhibits clustering and small distances between the vertices, has degree distribution that decays exponentially [4].

The notion of clustering formalizes the property that two nodes of a network that share a neighbor (for example, two individuals that have a common friend) are more likely to be joined by an edge (that is, to be friends of each other). In the context of social networks, sociologists have explained this phenomenon through the notion of homophily, which refers to the tendency of individuals to be related with similar individuals, e.g., having similar socioeconomic background or similar educational background. There have been numerous attempts to define models where clustering is present; see, for example, the work of [13] or that of [8] where this is combined with the general notion of inhomogeneity. In that context, clustering is planted in a sparse random graph. Also, it is even more rare to quantify clustering precisely (see, for example, the work of [5] on random intersection graphs). This is the case because the presence of clustering is the outcome of heavy dependencies between the edges of the random graphs and, in general, these are not easy to handle.

However, clustering is naturally present on random graphs that are created on a metric space, as is the case of a random geometric graph on the Euclidean plane. The theory of random geometric graphs was initiated by [18] in 1961 (see also [25]) and started taking its present form later by [20]. In its standard form, a geometric random graph is created as follows: $N$ points are sampled within a subset of $\mathbb{R}^{d}$ following a particular distribution (most usually this is the uniform distribution or the distribution of the point set of a Poisson point process) and any two of them are joined when their Euclidean distance is smaller than some threshold value which, in general, is a function of $N$. During the last two decades, this kind of random graphs was studied in depth by several researchers; see the monograph of [29] and the references therein. Numerous typical properties of such random graphs have been investigated, such as the chromatic number [24], Hamiltonicity [2], etc.

There is no particular reason why a random geometric graph on a Euclidean space would be intrinsically associated with the formation of a complex network. Real-world networks consist of heterogeneous nodes, which can be classified into groups. In turn, these groups can be classified into larger groups, which belong to larger subgroups, and so on. For example, if we consider the network of citations, whose set of nodes is the set of 
research articles, and there is a link from one article to another if one cites the other, there is a natural classification of the nodes according to the scientific fields each article belongs to (see, for example, [9]). In the case of the network of webpages, a similar classification can be considered in terms of the similarity between two webpages. That is, the more similar two webpages are, the more likely it is that there exists a hyperlink between them [26].

This classification can be approximated by tree like structures representing the hidden hierarchy of the network. The likeness to a tree suggests that, in fact, the geometry of this hierarchy is hyperbolic. One of the basic features of a hyperbolic space is that the volume growth is exponential, which is also the case, for example, when one considers a $k$-ary tree, that is, a rooted tree for which every vertex has $k$ children. Let us consider, for example, the Poincare unit disc model (which we will discuss in more detail in the next section). If we place the root of an infinite $k$-ary tree at the center of the disk, then the hyperbolic metric provides the necessary room to embed the tree into the disk so that every edge has unit length in the embedding.

Recently, a model was introduced [22] that implements this idea. In this model, a random network is created on the hyperbolic plane (we will see the detailed definition shortly). In particular, [22] determined the degree distribution for large degrees showing that it is scale free and its tail follows a power law, whose exponent is determined by some of the parameters of the model. Furthermore, they consider the clustering properties of the resulting random network. A numerical approach [22] suggests that the (local) clustering coefficient ${ }^{1}$ is positive and it is determined by one of the parameters of the model. In fact, as we will discuss in Section 1.3, this model corresponds to the sparse regime of random geometric graphs on the hyperbolic plane and, hence, is of independent interest within the theory of random geometric graphs.

This study investigates rigorously the presence of clustering in this model, through the notion of the clustering coefficient. Our first contribution is that we manage to determine exactly the value of the clustering coefficient as a function of the parameters of the model. More importantly, our results imply that, in fact, the exponent of the power law, the density of the random graph, and the amount of clustering can be tuned independently of each other, through the parameters of the random graph. Furthermore, we should point out that the clustering coefficient we consider is the so-called global clustering coefficient. Its calculation involves tight concentration bounds on the number of triangles in the random graph. Hence, our analysis initiates an approach to the small subgraph counting problem in these random graphs, which is among the central problems in the general theory of random graphs $[6,21]$ and of random geometric graphs [29].

We now proceed with the definition of the model of random geometric graphs on the hyperbolic plane.

\subsection{Random Geometric Graphs on the Hyperbolic Plane}

The most common representations of the hyperbolic plane are the upper-half plane representation $\{z: \Im z>0\}$ as well as the Poincaré unit disk which is simply the open disk of radius one, that is, $\left\{(u, v) \in \mathbb{R}^{2}: 1-u^{2}-v^{2}>0\right\}$. Both spaces are equipped with the hyperbolic metric; in the former case this is $\frac{d x^{2}+d y^{2}}{(\zeta y)^{2}}$, whereas in the latter this is $\frac{4}{\zeta^{2}} \frac{d u^{2}+d v^{2}}{\left(1-u^{2}-v^{2}\right)^{2}}$, where $\zeta$ is some positive real number. It can be shown that the (Gaussian)

${ }^{1}$ This is defined as the average density of the neighborhoods of the vertices. 
curvature in both cases is equal to $-\zeta^{2}$ and the two spaces are isometric, i.e., there is a bijection between the two spaces that preserves (hyperbolic) distances. In fact, there are more representations of the 2-dimensional hyperbolic space of curvature $-\zeta^{2}$, which are isometrically equivalent to the above two. We will denote by $\mathbb{H}_{\zeta}^{2}$ the class of these spaces.

In this work, following the definitions in [22], we will use the native representation of $\mathbb{H}_{\zeta}^{2}$. Under this representation, the ground space of $\mathbb{H}_{\zeta}^{2}$ is $\mathbb{R}^{2}$, and every point $x \in \mathbb{R}^{2}$ whose polar coordinates are $(r, \theta)$ has hyperbolic distance from the origin equal to $r$. More precisely, the native representation can be viewed as a mapping of the Poincaré unit disk to $\mathbb{R}^{2}$, in which the origin of the unit disk is mapped to the origin of $\mathbb{R}^{2}$, and every point $v$ in the Poincaré disk is mapped to a point $v^{\prime} \in \mathbb{R}^{2}$, where $v^{\prime}=(r, \theta)$ in polar coordinates. More specifically, $r$ is the hyperbolic distance of $v$ from the origin of the Poincare disk and $\theta$ is its angle.

Also, a circle of radius $r$ around the origin has length equal to $\frac{2 \pi}{\zeta} \sinh \zeta r$ and area equal to $\frac{2 \pi}{\zeta^{2}}(\cosh \zeta r-1)$.

We are now ready to give the definitions of the two basic models introduced in [22]. Consider the native representation of the hyperbolic plane of curvature $K=-\zeta^{2}$, for some $\zeta>0$. For some constant $v>0$, we let $N=v e^{\zeta R / 2}$; thus, $R$ grows logarithmically as a function of $N$. We shall explain the role of $v$ shortly. Let $V_{N}=\left\{v_{1}, \ldots, v_{N}\right\}$ be the set of vertices of the random graphs, where $v_{i}$ is a random point on the disk of radius $R$ centered at the origin $O$, which we denote by $\mathcal{D}_{R}$. In other words, the vertex set $V_{N}$ is a set of i.i.d. random variables that take values on $\mathcal{D}_{R}$.

Their distribution is as follows. Assume that $u$ has polar coordinates $(r, \theta)$. The angle $\theta$ is uniformly distributed in $(0,2 \pi]$ and the probability density function of $r$, which we denote by $\rho_{N}(r)$, is determined by a parameter $\alpha>0$ and is equal to

$$
\rho_{N}(r)=\left\{\begin{array}{ll}
\alpha \frac{\sinh \alpha r}{\cosh \alpha R-1}, & \text { if } 0 \leq r \leq R \\
0, & \text { otherwise }
\end{array} .\right.
$$

Note that when $\alpha=\zeta$, this is simply the uniform distribution.

An alternative way to define this distribution is as follows. Consider $\mathbb{H}_{\alpha}^{2}$ and select arbitrarily a point $O^{\prime}$ on this space, which will be assumed to be its origin. Consider the disk $\mathcal{D}_{R}^{\prime}$ of radius $R$ around $O^{\prime}$ and select $N$ points within $\mathcal{D}_{R}^{\prime}$ uniformly at random. Subsequently, the selected points are projected onto $\mathcal{D}_{R}$, preserving their polar coordinates. The projections of these points, which we will be denoting by $\mathrm{V}_{N}$, will be the vertex set of the random graph. We will also be treating the vertices as points in the hyperbolic space indistinguishably.

More precisely, given $\alpha, \zeta>0$, we consider two different Poincaré disk representations using two different metrics: one being $(d s)^{2}:=\frac{4}{\zeta^{2}} \frac{d u^{2}+d v^{2}}{\left(1-u^{2}-v^{2}\right)^{2}}$, and the other $\left(d s^{\prime}\right)^{2}:=\frac{4}{\alpha^{2}} \frac{d u^{2}+d v^{2}}{\left(1-u^{2}-v^{2}\right)^{2}}$. Subsequently, we consider the disk around the origin with (hyperbolic) radius $R$ in the metric $\left(d s^{\prime}\right)^{2}$. There, we select the $N=v e^{\zeta R / 2}$ vertices of $V_{N}$ uniformly at random. Thereafter, we project these vertices onto the disk of (hyperbolic) radius $R$ under the metric $(d s)^{2}$, on which we create the random graph.

Note that the curvature in this case determines the rate of growth of the space. Hence, when $\alpha<\zeta$, the $N$ points are distributed on a disk (namely $\mathcal{D}_{R}^{\prime}$ ), which has smaller area compared to $\mathcal{D}_{R}$. This naturally increases the density of those points that are located closer 

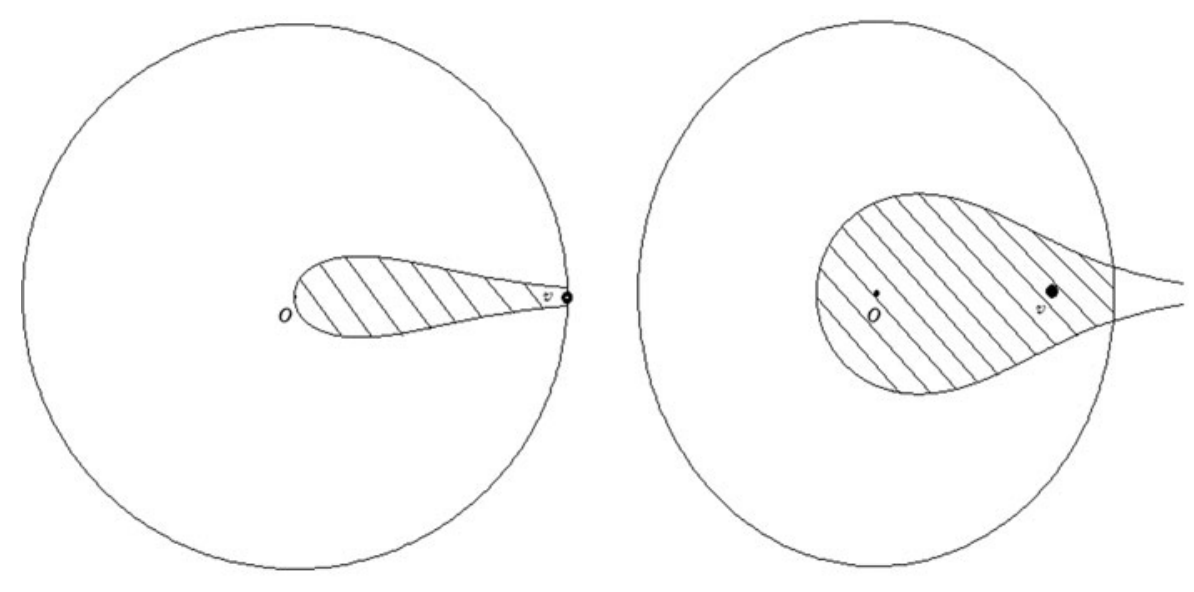

Figure 1 The disk of radius $R$ around $v$ in $\mathcal{D}_{R}$.

to the origin. Similarly, when $\alpha>\zeta$, the area of the disk $\mathcal{D}_{R}^{\prime}$ is larger than that of $\mathcal{D}_{R}$, and most of the $N$ points are significantly more likely to be located near the boundary of $\mathcal{D}_{R}^{\prime}$, because of the exponential growth of the volume.

Given the set $\mathrm{V}_{N}$ on $\mathcal{D}_{R}$ we define the following two models of random graphs.

1. The disk model: this model is the most commonly studied in the theory of random geometric graphs on Euclidean spaces. We join two vertices if they are within (hyperbolic) distance $R$ from each other. Figure 1 illustrates a disk of radius $R$ around a vertex $v \in \mathcal{D}_{R}$.

2. The binomial model: we join any two distinct vertices $u, v$ with probability

$$
p_{u, v}=\frac{1}{\exp \left(\beta \frac{\zeta}{2}(d(u, v)-R)\right)+1},
$$

independently of every other pair, where $\beta>0$ is fixed and $d(u, v)$ is the hyperbolic distance between $u$ and $v$. We denote the resulting random graph by $\mathcal{G}(N ; \zeta, \alpha, \beta, \nu)$.

The binomial model is in some sense a soft version of the disk model. In the latter, two vertices become adjacent if and only if their hyperbolic distance is at most $R$. This is approximately the case in the former model. If $d(u, v)=(1+\delta) R$, where $\delta>0$ is some constant, then $p_{u, v} \rightarrow 0$, whereas if $d(u, v)=(1-\delta) R$, then $p_{u, v} \rightarrow 1$, as $N \rightarrow \infty$. (Recall that $R \rightarrow \infty$ as $N \rightarrow \infty$.)

Also, the disk model can be viewed as a limiting case of the binomial model as $\beta \rightarrow \infty$. Assume that the positions of the vertices in $\mathcal{D}_{R}$ have been realized. If $u, v \in V_{N}$ are such that $d(u, v)<R$, then when $\beta \rightarrow \infty$ the probability that $u$ and $v$ are adjacent tends to 1 ; however, if $d(u, v)>R$, then this probability converges to 0 as $\beta$ grows.

The binomial model has also a statistical-mechanical interpretation where by the parameter $\beta$ can be seen as the inverse temperature of a fermionic system where particles correspond to the edges of the random graph; see [22] for a detailed discussion.

An argument is provided [22] that indicates in both models the degree distribution has a power-law tail with an exponent that is equal to $2 \alpha / \zeta+1$. Hence, when $0<\zeta / \alpha<2$, any exponent greater than 2 can be realized. This has been shown rigorously, for the disc 
model [19], and by [17], for the binomial model. In the latter case, the average degree of a vertex depends on all four parameters of the model. For the disk model in particular, having fixed $\zeta$ and $\alpha$, which determine the exponent of the power law, the parameter $v$ determines the average degree. In the binomial model, there is an additional dependence on $\beta$. However, our main results show that clustering does not depend on $v$. Therefore, in the binomial model the "amount" of clustering and the average degree can be tuned independently.

It has been shown [17] that $\beta=1$ is a critical point around which there is a transition on the density of $\mathcal{G}(N ; \zeta, \alpha, \beta, v)$. When $\beta \leq 1$, the average degree of the random graph grows at least logarithmically in $N$. The case where $\beta>1$ and $0<\zeta / \alpha<2$ becomes of particular interest as the random graph obtains the characteristics that are ascribed to complex networks. More specifically, in this regime $\mathcal{G}(N ; \zeta, \alpha, \beta, v)$ has a constant (i.e., not depending on $N$ ) average degree that depends on $v, \zeta, \alpha$, and $\beta$, whereas the degree distribution follows the tail of a power law with exponent $2 \alpha / \zeta+1$. Theorem 1.2 in [17] implies that, for $\beta>1$, the average degree is asymptotic to $2 v\left(\frac{2 \alpha}{2 \alpha-\zeta}\right)^{2} \frac{1}{\beta} \arcsin \left(\frac{\pi}{\beta}\right)$. In this article, we show that the (global) clustering coefficient is only a function of $\zeta, \alpha$, and $\beta$.

\subsection{Notation}

Let $\left\{X_{N}\right\}_{N \in \mathbb{N}}$ be a sequence of real-valued random variables on a sequence of probability spaces $\left\{\left(\Omega_{N}, \mathbb{P}_{N}\right)\right\}_{N \in \mathbb{N}}$, and let $\left\{a_{N}\right\}_{N \in \mathbb{N}}$ be a sequence of real numbers that tends to infinity as $N \rightarrow \infty$.

We write $X_{N}=o_{p}\left(a_{N}\right)$, if $\left|X_{N}\right| / a_{N}$ converges to 0 in probability. That is, for any $\varepsilon>0$, we have $\mathbb{P}_{N}\left(\left|X_{N} / a_{N}\right|>\varepsilon\right) \rightarrow 0$ as $N \rightarrow \infty$. Additionally, we write $X_{N}=\Theta_{C}\left(a_{N}\right)$ if there exist positive real numbers $C_{1}, C_{2}$ such that we have $\mathbb{P}\left(C_{1} a_{N} \leq\left|X_{N}\right| \leq C_{2} a_{N}\right)=$ $1-o(1)$. Finally, if $\mathcal{E}_{N}$ is a measurable subset of $\Omega_{N}$, for any $N \in \mathbb{N}$, we say that the sequence $\left\{\mathcal{E}_{N}\right\}_{N \in \mathbb{N}}$ occurs asymptotically almost surely (a.a.s.) if $\mathbb{P}\left(\mathcal{E}_{N}\right)=1-o(1)$, as $N \rightarrow \infty$. However, with a slight abuse of terminology, we will say that an event occurs a.a.s., implicitly referring to a sequence of events.

For two functions $f, g: \mathbb{N} \rightarrow \mathbb{R}$ we write $f(N) \ll g(N)$ if $f(N) / g(N) \rightarrow 0$ as $N \rightarrow \infty$. Similarly, we will write $f(N) \asymp g(N)$, meaning that there are positive constants $c_{1}, c_{2}$ such that for all $N \in \mathbb{N}$ we have $c_{1} g(N) \leq f(N) \leq c_{2} g(N)$. Analogously, we write $f(N) \lesssim g(N)$ (resp. $f(N) \gtrsim g(N))$ if there is a positive constant $c$ such that for all $N \in \mathbb{N}$ we have $f(N) \leq c g(N)$ (resp. $f(N) \geq c g(N)$ ). These notions could have been expressed through the standard Landau notation, but we chose to express them as we have in order to make our calculations more readable.

Finally, we write $X_{N} \lesssim g(N)$ a.a.s., if there is a positive constant $c$ such that $X_{N} \leq c g(N)$ a.a.s. An analogous interpretation is used for $X_{N} \gtrsim g(N)$ a.a.s.

\subsection{Some Geometric Aspects of the Two Models}

The disc model on the hyperbolic plane can also be viewed within the framework of random geometric graphs. Within this framework, the disk model may be defined for any threshold distance $r_{N}$ and not merely for threshold distance equal to $R$. However, taking only $r_{N}=R$ yields a random graph with constant average degree that is bounded away from 0 . More specifically, for any $\delta \in(0,1)$, if $r_{N}=(1-\delta) R$, then the resulting random graph becomes rather trivial and most vertices have no neighbors. To the contrary, 
if $r_{N}=(1+\delta) R$, the resulting random graph becomes too dense and its average degree grows polynomially fast in $N$.

The proof of these observations relies on the following lemma, which provides a characterization of what it means for two points $u, v$ to have $d(u, v) \leq(1+\delta) R$, for $\delta \in(-1,1)$, in terms of their relative angle, which we denote by $\theta_{u, v}$. For this lemma, we need the notion of the type of a vertex. For a vertex $v \in \mathrm{V}_{N}$, if $r_{v}$ is the distance of $v$ from the origin, that is, the radius of $v$, then we set $t_{v}=R-r_{v}$; we call this quantity the type of vertex $v$.

Lemma 1.1. Let $\delta \in(-1,1)$ be a real number. For any $\varepsilon>0$ there exists an $N_{0}>0$ and a $c_{0}>0$ such that for any $N>N_{0}$ and $u, v \in \mathcal{D}_{R}$ with $t_{u}+t_{v}<(1-|\delta|) R-c_{0}$ the following hold.

- If $\theta_{u, v}<2(1-\varepsilon) \exp \left(\frac{\xi}{2}\left(t_{u}+t_{v}-(1-\delta) R\right)\right)$, then $d(u, v)<(1+\delta) R$.

- If $\theta_{u, v}>2(1+\varepsilon) \exp \left(\frac{\xi}{2}\left(t_{u}+t_{v}-(1-\delta) R\right)\right)$, then $d(u, v)>(1+\delta) R$.

The proof of this lemma can be found in Appendix B.

Let us consider temporarily the (modified) disk model, for which we assume that two vertices are joined precisely when their hyperbolic distance is at most $(1+\delta) R$. Let $u \in \mathrm{V}_{N}$ be a vertex and assume that $t_{u}<C$ (in fact, by Claim 3.4, if $C$ is large enough, then most vertices will satisfy this). We will show that if $\delta<0$, then the expected degree of $u$, in fact, tends to 0 . Let us consider a simple case wherein $\delta$ satisfies $\frac{\zeta}{2 \alpha}<1-|\delta|<1$. As we will see later (Corollary 3.5), a.a.s. there are no vertices of type much larger than $\frac{\zeta}{2 \alpha} R$. Hence, since $t_{u}<C$, if $N$ is sufficiently large, then we have $\frac{\zeta}{2 \alpha} R<(1-|\delta|) R-t_{u}-c_{0}$. By Lemma 1.1, the probability that a vertex $v$ has type at most $\frac{\zeta}{2 \alpha} R$ and it is adjacent to $u$ (that is, its hyperbolic distance from $u$ is at most $(1+\delta) R)$ is proportional to

$$
e^{\frac{\zeta}{2}\left(t_{u}+t_{v}-(1-\delta) R\right)} / \pi
$$

If we average this over $t_{v}$, and denote by $u \sim v$ the relation of neighborhood between the two vertices $u$ and $v$, we obtain

$$
\begin{aligned}
\operatorname{Pr}\left[u \sim v \mid t_{u}\right] & \asymp \frac{e^{\zeta t_{u} / 2}}{e^{\frac{\zeta}{2}(1-\delta) R}} \int_{0}^{\frac{\zeta}{2 \alpha} R} e^{\zeta t_{v} / 2} \frac{\alpha \sinh \left(\alpha\left(R-t_{v}\right)\right)}{\cosh (\alpha R)-1} d t_{v} \\
& \lesssim \frac{e^{\zeta t_{u} / 2}}{e^{\frac{\zeta}{2}(1-\delta) R}} \int_{0}^{R} e^{\zeta t_{v} / 2} \frac{e^{\alpha\left(R-t_{v}\right)}}{\cosh (\alpha R)-1} d t_{v} \\
& \asymp \frac{e^{\zeta t_{u} / 2}}{e^{\frac{\zeta}{2}(1-\delta) R}} \int_{0}^{R} e^{(\zeta / 2-\alpha) t_{v}} d t_{v} \stackrel{0<\zeta / \alpha<2}{\asymp} \frac{e^{\zeta t_{u} / 2}}{N^{1-\delta}} \stackrel{\delta \leq 0}{=} o\left(\frac{1}{N}\right) .
\end{aligned}
$$

Hence, the probability that there is such a vertex is $o(1)$. Markov's inequality implies that, with high probability, most vertices will have no neighbors.

A similar calculation can actually show that this probability is $\Omega\left(\frac{e^{\zeta t_{u} / 2}}{N^{1-\delta}}\right)$. Thereby, if $0<\delta<1$, then the expected degree of $u$ is of order $N^{\delta}$. A more detailed argument can show that the resulting random graph is too dense in the sense that the number of edges is no longer proportional to the number of vertices but grows much faster than that.

Of course, to create a random graph with constant average degree, a different scaling could have been chosen. For example, one could choose the threshold distance to be a 
positive constant $r$. In that case, the hyperbolic law of cosines (see Fact 3.3) implies that two typical vertices, which are close to the boundary of $\mathcal{D}_{R}$, would be within distance $r$ if their relative angle is of order $e^{-\zeta R}$. In other words, the probability that two typical vertices are adjacent would be proportional to $e^{-\zeta R}$. Hence, such a vertex would have a constant expected degree if the total number of vertices is scaled as $e^{\zeta R}$ (which is the volume of $\mathcal{D}_{R}$ ). However, such a choice would create a random model, which is not suitable for complex networks. For example, the degree distribution would lose its heavy tail. In particular, the vertices that are close to the center would not act as the high-degree hubs that hold the network together. Such vertices, in general, are fairly important because they give rise to short typical distances.

This heuristic also suggests that when $\delta=0$, the expected degree of vertex $u$ is proportional to $e^{\zeta t_{u} / 2}$. In the present article, we focus on this case. As we shall see later, in Claim 3.4, the type of a vertex $u$ follows approximately the exponential distribution $e^{-\alpha t_{u}}$. This implies that $e^{\zeta t_{u} / 2}$ follows a power-law degree distribution with exponent equal to $2 \alpha / \zeta+1$. This is precisely the effect of selecting the vertices on the hyperbolic plane of curvature $-\alpha^{2}$ and thereafter projecting them onto the plane of curvature $-\zeta^{2}$. The parameter $\alpha$ comes up as the parameter of the exponential distribution that (partially) determines the position of a vertex. One may view the quantity $e^{\zeta t_{u} / 2}$ as the weight of vertex $u$. Let us point out that this is precisely the degree distribution in the ChungLu model $[10,11]$, in which vertices have associated weights that follow a power-law distribution and any two vertices are joined with probability proportional to the product of these weights independently of the other pairs. In our context, however, due to the underlying geometry, dependencies are present.

\subsection{The Clustering Coefficient}

The theme of this work is the study of clustering in $\mathcal{G}(N ; \zeta, \alpha, \beta, v)$. The notion of clustering was introduced as a measure of the "cliquishness of a typical neighborhood" [30]. More specifically, it measures the expected density of the neighbourhood of a randomly chosen vertex. In the context of biological or social networks, this is expressed as the likelihood of two vertices that have a common neighbor to be joined with each other. More specifically, for each vertex $v$ of a graph, the local clustering coefficient $C(v)$ is defined to be the density of the neighborhood of $v$. In [30], the clustering coefficient of a graph $G$, which we denote by $C_{1}(G)$, is defined as the average of the local clustering coefficients over all vertices of $G$. The clustering coefficient $C_{1}(\mathcal{G}(N ; \zeta, \alpha, \beta, v))$, as a function of $\beta$ is discussed in [22], where simulations and heuristic calculations indicate that $C_{1}$ can be tuned by $\beta$. For the disk model, [19] have shown rigorously that this quantity is asymptotically with high probability bounded away from 0 when $0<\zeta / \alpha<2$.

As mentioned, there is significant experimental evidence that shows that many networks that arise in applications have degree distributions that follow a power law, usually with exponent between 2 and 3 (cf. [1]). Also, such networks are typically sparse with only a few nodes of very high degree, which are the hubs of the network. Thus, in the regime where $\beta>1$ and $1<\zeta / \alpha<2$, the random graph $\mathcal{G}(N ; \zeta, \alpha, \beta, v)$ appears to exhibit these characteristics. In this work, we explore further the potential of this random graph model as a suitable model for complex networks focusing on the notion of global clustering and how this is determined by the parameters of the model.

A first attempt to define this notion was made by [23], but it was rediscovered more recently by [28]. Given a graph $G$, we let $T=T(G)$ be the number of triangles of $G$ and let 
$\Lambda=\Lambda(G)$ denote the number of incomplete triangles of $G$; this is simply the number of the (not necessarily induced) paths having length 2 . Then the global clustering coefficient $C_{2}(G)$ of a graph $G$ is defined as

$$
C_{2}(G):=\frac{3 T(G)}{\Lambda(G)}
$$

This parameter measures the likelihood that two vertices that share a neighbor are themselves adjacent.

The present work has to do with the value of $C_{2}(\mathcal{G}(N ; \zeta, \alpha, \beta, v))$. Our results show exactly how clustering can be tuned by the parameters $\beta, \zeta$, and $\alpha$ only. More precisely, our main result states that this undergoes an abrupt change as $\beta$ crosses the critical value 1 .

Theorem 1.2. Let $0<\zeta / \alpha<2$. If $\beta>1$, then

$$
C_{2}(\mathcal{G}(N ; \zeta, \alpha, \beta, \nu)) \stackrel{p}{\rightarrow}\left\{\begin{array}{ll}
L_{\infty}(\beta, \zeta, \alpha), & \text { if } 0<\zeta / \alpha<1 \\
0, & \text { if } 1 \leq \zeta / \alpha<2
\end{array},\right.
$$

where

$$
\begin{aligned}
& L_{\infty}(\beta, \zeta, \alpha) \\
& \quad=\frac{3}{2} \frac{(\zeta-2 \alpha)^{2}(\alpha-\zeta)}{\left(\pi C_{\beta}\right)^{2}} \int_{[0, \infty)^{3}} e^{\frac{\zeta}{2}\left(t_{u}+t_{v}\right)+\zeta t_{w}} g_{t_{u}, t_{v}, t_{w}}(\beta, \zeta) e^{-\alpha\left(t_{u}+t_{v}+t_{w}\right)} d t_{u} d t_{v} d t_{w} \in(0,1],
\end{aligned}
$$

with

$$
g_{t_{u}, t_{v}, t_{w}}(\beta, \zeta)=\int_{[0, \infty)^{2}} \frac{1}{z_{1}^{\beta}+1} \frac{1}{z_{2}^{\beta}+1} \frac{1}{\left(e^{\frac{\zeta}{2}\left(t_{w}-t_{v}\right)} z_{1}+e^{\frac{\zeta}{2}\left(t_{w}-t_{u}\right)} z_{2}\right)^{\beta}+1} d z_{1} d z_{2}
$$

and $C_{\beta}:=\frac{2}{\beta \sin (\pi / \beta)}$.

If $\beta \leq 1$, then

$$
C_{2}(\mathcal{G}(N ; \zeta, \alpha, \beta, v)) \stackrel{p}{\rightarrow} 0
$$

Our results complement those of [22], whose estimates suggest that $C_{1}$ is tuned by $\beta$. From a different point of view, the global clustering coefficient sometimes gives more accurate information about the network [7]; for example, a graph that is a double star with two adjacent centers 1 and 2, where each of them is joined with $n-1$ other vertices [7]. Clearly this graph exhibits no clustering as it is a tree, nevertheless $C_{1}$ approaches 1 as $n$ grows, whereas $C_{2}$ vanishes. Furthermore, the study of $C_{2}$ is associated with subgraph counting, which is a central topic in the theory of random graphs. Our analysis may need significant modification in order to be applied to $C_{1}$.

The fact that the global clustering coefficient asymptotically vanishes when $\zeta / \alpha \geq 1$ is because when $\zeta / \alpha$ crosses 1 , vertices of very high degree appear, which incur an abrupt increase in the number of incomplete triangles, with no similar increase on the number of triangles. In other words, vertices of high degree do not create many triangles. In our context, this is the case because the edges that are incident to a vertex of high degree are spread out and, as a result of this, most of their other endvertices are not close enough to 
each other. The same phenomenon appears in very different contexts: in random intersection graphs [5], as well as in a spatial model of preferential attachment [27]. In both articles, it is shown that the global clustering coefficient in that model is positive if and only if the second moment of the degree sequence is finite. This is found to be [16] a natural feature of social networks: two neighbors of a very high-degree vertex are somewhat less likely to be adjacent to each other than if they were neighbors of a low-degree vertex.

Recall that for a vertex $u \in \mathrm{V}_{N}$, its type $t_{u}$ is defined to be equal to $R-r_{u}$, where $r_{u}$ is the radius (i.e., its distance from the origin) of $u$ in $\mathcal{D}_{R}$. When $1 \leq \zeta / \alpha<2$, vertices of type larger than $R / 2$ appear, which affect the tail of the degree sequence of $\mathcal{G}(N ; \zeta, \alpha, \beta, v)$. For $\beta>1$, it was shown [17] that when $1 \leq \zeta / \alpha<2$, the degree sequence follows approximately a power law with exponent in $(2,3]$. More precisely, asymptotically as $N$ grows, the degree of a vertex $u \in \mathrm{V}_{N}$ conditional on its type follows a Poisson distribution with parameter equal to $K e^{\zeta t_{u} / 2}$, where $K=K(\zeta, \alpha, \beta, v)>0$. Also, a simple calculation reveals that most vertices have small types. This is made more precise in Claim 3.4, where we show that the types have approximately exponential density $e^{-\alpha t}$. In fact, when $\zeta / \alpha<1$, a.a.s. all vertices have type less than $R / 2$ (cf. Corollary 3.5 ).

Let us consider more closely, for example, the case $\zeta=\alpha$, where the $N$ points are uniformly distributed on $\mathcal{D}_{R}$. In this case, the type of a vertex $u$ is approximately exponentially distributed with density $\zeta e^{-\zeta t_{u}}$. Hence, there are about $N e^{-\zeta(R / 2-\omega(N))} \asymp$ $e^{\zeta \omega(N)}$ vertices of type between $R / 2-\omega(N)$ and $R / 2+\omega(N)$; here, $\omega(N)$ is assumed to be a slowly growing function. Now, each of these vertices has degree that is at least $e^{\frac{\zeta}{2}\left(\frac{R}{2}-\omega(N)\right)}=N^{1 / 2} e^{-\zeta \omega(N) / 2}$. Therefore, these vertices' contribution to $\Lambda$ is at least $e^{\zeta \omega(N)} \times$ $\left(N^{1 / 2} e^{-\zeta \omega(N) / 2}\right)^{2}=N$.

Now, if vertex $u$ is of type less than $R / 2-\omega(N)$, its contribution to $\Lambda$ in expectation is proportional to

$$
\int_{0}^{R / 2-\omega(N)}\left(e^{\zeta t_{u} / 2}\right)^{2} e^{-\zeta t_{u}} d t_{u} \asymp R .
$$

Because most vertices are indeed of type less than $R / 2-\omega(N)$, it follows that these vertices contribute $R N$ on average to $\Lambda$.

However, the number of triangles these vertices contribute is asymptotically much smaller. Recall that for any two vertices $u, v$ the probability that these are adjacent is bounded away from 0 when $d(u, v)<R$. Consider three vertices $w, u, v$, which, without loss of generality they satisfy $t_{v}<t_{u}<t_{w}<R / 2-\omega(N)$. As we shall see later (cf. Fact 3.3) having $d(u, v)<R$ is almost equivalent to having $\theta_{u, v} \lesssim e^{\frac{\zeta}{2}\left(t_{u}+t_{v}-R\right)}$. Because the relative angle between $u$ and $v$ is uniformly distributed in $[0, \pi]$, it turns out that the probability that $u$ is adjacent to $w$ is proportional to $e^{\frac{5}{2}\left(t_{w}+t_{u}-R\right)}$; similarly, the probability that $v$ is adjacent to $w$ is proportional to $e^{\frac{\zeta}{2}\left(t_{w}+t_{v}-R\right)}$. Note that these events are independent. Now, conditional on these events, the relative angle between $u$ and $w$ is approximately uniformly distributed in an interval of length $e^{\frac{\zeta}{2}\left(t_{w}+t_{u}-R\right)}$. Similarly, the relative angle between $v$ and $w$ is approximately uniformly distributed in an interval of length $e^{\frac{\zeta}{2}\left(t_{w}+t_{v}-R\right)}$. Hence, the (conditional) probability that $u$ is adjacent to $v$ is bounded by a quantity that is proportional to $e^{\frac{\zeta}{2}\left(t_{u}+t_{v}\right)} / e^{\frac{\zeta}{2}\left(t_{v}+t_{w}\right)}=e^{\frac{\zeta}{2}\left(t_{u}-t_{w}\right)}$. This implies that the probability that $u, v$, and $w$ form a triangle is proportional to $e^{\frac{\zeta}{2} t_{w}+\zeta t_{u}+\frac{\zeta}{2} t_{v}} / N^{2}$. Averaging over the types of these vertices we have

$$
\frac{1}{N^{2}} \int_{0}^{R / 2-\omega(N)} \int_{0}^{t_{w}} \int_{0}^{t_{u}} e^{\frac{\zeta}{2} t_{w}+\zeta t_{u}+\frac{\zeta}{2} t_{v}-\zeta\left(t_{v}+t_{u}+t_{w}\right)} d t_{v} d t_{u} d t_{w} \asymp \frac{1}{N^{2}} .
$$


Hence, the expected number of triangles that have all their vertices of type at most $R / 2-$ $\omega(N)$ is only proportional to $N$. Note that if we take $\alpha>\zeta$, then the previous expression is still proportional to $N$, whereas (1.3) becomes asymptotically constant, giving contribution to $\Lambda$ that is also proportional to $N$. This makes the clustering coefficient be bounded away from 0 when $\zeta / \alpha<1$. Our analysis will make these heuristics rigorous and generalize them for all values of $\zeta / \alpha<2$ and $\beta>0$.

It turns out that the situation is somewhat different if we do not take into consideration high-degree vertices (or, equivalently, vertices that have large type). For any fixed $t>0$, we will consider the global clustering coefficient of the subgraph of $\mathcal{G}(N ; \zeta, \alpha, \beta, \nu)$ that is induced by those vertices that have type at most $t$. We will denote this by $\widehat{C_{2}}(t)$. We will show that when $\beta>1$, then for all $0<\zeta / \alpha<2$, the quantity $\widehat{C_{2}}(t)$ remains bounded away from 0 with high probability. Moreover, we determine its dependence on $\zeta, \alpha, \beta$.

Theorem 1.3. Let $0<\zeta / \alpha<2$ and let $t>0$ be fixed. If $\beta>1$, then

$$
\widehat{C_{2}}(t) \stackrel{p}{\rightarrow} L(t ; \beta, \zeta, \alpha)
$$

where

$$
L(t ; \beta, \zeta, \alpha):=\frac{6 \int_{[0, t)^{3}} e^{\frac{\zeta}{2}\left(t_{u}+t_{v}\right)+\zeta t_{w}} g_{t_{u}, t_{v}, t_{w}}(\beta, \zeta) e^{-\alpha\left(t_{u}+t_{v}+t_{w}\right)} d t_{u} d t_{v} d t_{w}}{\left(\pi C_{\beta}\right)^{2} \int_{[0, t)^{3}} e^{\frac{\zeta}{2}\left(t_{u}+t_{v}\right)+\zeta t_{w}} e^{-\alpha\left(t_{u}+t_{v}+t_{w}\right)} d t_{u} d t_{v} d t_{w}},
$$

where $g_{t_{u}, t_{v}, t_{w}}(\beta, \zeta)$ and $C_{\beta}$ are as in Theorem 1.2.

The most involved part of the proofs, which may be of independent interest, has to do with counting triangles in $\mathcal{G}(N ; \zeta, \alpha, \beta, \nu)$, that is, with estimating $T(\mathcal{G}(N ; \zeta, \alpha, \beta, \nu))$. In fact, most of our effort is devoted to the calculation of the probability that three vertices form a triangle. Thereafter, a second moment argument, together with the fact that the degree of high-type vertices is concentrated around its expected value, implies that $T(\mathcal{G}(N ; \zeta, \alpha, \beta, v))$ is close to its expected value.

This article is organized as follows. In Section 2 we state a series of results that imply Theorem 1.2. Section 5 is mainly devoted to showing that the random variables counting the number of typical incomplete and complete triangles (i.e., whose vertices have type at most $R / 2-\omega(N)$, for a suitable growing function $\omega(N))$ are concentrated around their expected values. We find precise (asymptotic) expressions for these values in Sections 6 and 7, (where we deduce Theorem 1.3), and 7. Section 8 takes care of the atypical (i.e., nontypical) case, together with the calculations shown in Appendix A.

\section{TRIANGLES AND CONCENTRATION: PROOF OF THEOREM 1.2}

The main ingredient of the proofs of Theorems 1.2 and 1.3 is a collection of concentration results with regard to the number of triangles as well as the number of incomplete triangles. For a function $\omega: \mathbb{N} \rightarrow \mathbb{N}$ such that $\omega(N) \rightarrow \infty$ as $N \rightarrow \infty$, we call a vertex $u$ typical if $t_{u} \leq R / 2-\omega(N)$. The function $\omega$ grows slowly enough so that our calculations work. For example, we may set $\omega(N)=\ln \ln \ln (N)$. We consider two classes of triangles: those that consist of typical vertices and those that contain at least one vertex that is not typical. 
In particular, we introduce the random variables $\widehat{T}$ and $\widehat{\Lambda}$, which denote the numbers of triangles and incomplete triangles, respectively, with all their three vertices being typical. We also introduce the random variables $\widetilde{T}$ and $\widetilde{\Lambda}$, which denote the numbers of triangles and incomplete triangles, respectively, which have at least one vertex that is not typical, or, as we shall be saying, atypical. Hence,

$$
T=\widehat{T}+\widetilde{T}, \text { and } \Lambda=\widehat{\Lambda}+\widetilde{\Lambda}
$$

We now give a series of propositions that describe how the expected values of $\widehat{T}$ and $\widehat{\Lambda}$ vary according to the parameters $\zeta, \alpha$, and $\beta$.

Proposition 2.1. Let $0<\zeta / \alpha<2$. Then the following hold:

(i) for $\beta>1$

$$
\mathbb{E}(\widehat{\Lambda}) \asymp \begin{cases}N, & \text { if } \frac{\zeta}{\alpha}<1 \\ R N, & \text { if } \frac{\zeta}{\alpha}=1 \\ N^{2-\alpha / \zeta} e^{-(\zeta-\alpha) \omega(N)}, & \text { if } \frac{\zeta}{\alpha}>1\end{cases}
$$

(ii) for $\beta=1$

$$
\mathbb{E}(\widehat{\Lambda}) \asymp \begin{cases}R^{2} N, & \text { if } \frac{\zeta}{\alpha}<1 \\ R^{3} N, & \text { if } \frac{\zeta}{\alpha}=1 \\ R^{2} N^{2-\alpha / \zeta} e^{-(\zeta-\alpha) \omega(N)}, & \text { if } \frac{\zeta}{\alpha}>1\end{cases}
$$

(iii) for $\beta<1$

$$
\mathbb{E}(\widehat{\Lambda}) \asymp \begin{cases}N^{3-2 \beta}, & \text { if } \frac{\beta \zeta}{\alpha}<1 \\ R N^{3-2 \beta}, & \text { if } \frac{\beta \zeta}{\alpha}=1 \\ N^{3-\beta-\alpha / \zeta} e^{(\alpha-\beta \zeta) \omega(N)}, & \text { if } \frac{\beta \zeta}{\alpha}>1\end{cases}
$$

The following proposition is the counterpart of Proposition 2.1 for triangles.

Proposition 2.2. Let $0<\zeta / \alpha<2$. Then the following hold:

(i) for $\beta>1$

$$
\mathbb{E}(\widehat{T}) \asymp\left\{\begin{array}{ll}
\mathbb{E}(\widehat{\Lambda}), & \text { if } \frac{\zeta}{\alpha}<1 \\
o(\mathbb{E}(\widehat{\Lambda})), & \text { if } \frac{\zeta}{\alpha} \geq 1
\end{array} .\right.
$$

(ii) for $\beta \leq 1$

$$
\mathbb{E}(\widehat{T})=o(\mathbb{E}(\widehat{\Lambda}))
$$

The following proposition states that the random variables $\widehat{\Lambda}$ and $\widehat{T}$ are concentrated around their expected values $\mathbb{E}(\widehat{\Lambda})$ and $\mathbb{E}(\widehat{T})$, respectively. 
Proposition 2.3. Let $0<\zeta / \alpha<2$. Then, for all $\beta>0$,

$$
\widehat{\Lambda}=\mathbb{E}(\widehat{\Lambda})\left(1+o_{p}(1)\right)
$$

and for $0<\zeta / \alpha<1$ and $\beta>1$,

$$
\widehat{T}=\mathbb{E}(\widehat{T})\left(1+o_{p}(1)\right) .
$$

The next result deals with the number of atypical triangles. Note that, since each triangle contains three incomplete triangles, we always have $\widetilde{T} \leq \widetilde{\Lambda} / 3$.

Proposition 2.4. For $0<\zeta / \alpha<2$ and any $\beta>0$, we have:

if $\zeta / \alpha<1$, then

$$
\widetilde{T} \leq \frac{1}{3} \widetilde{\Lambda}=o_{p}(1)
$$

thus, a.a.s. we have $\widetilde{T}=\widetilde{\Lambda}=0$.

If $\zeta / \alpha \geq 1$, then

$$
\widetilde{T}=o_{p}(\mathbb{E}(\widehat{\Lambda}))
$$

\subsection{Proof of Theorem 1.2}

We begin with the case in which $0<\zeta / \alpha<1$ and $\beta>1$. From Proposition 2.3, it follows that $\widehat{T}$ and $\widehat{\Lambda}$ are concentrated around their expected values $\mathbb{E}(\widehat{T})$ and $\mathbb{E}(\widehat{\Lambda})$, respectively. Also, the first part of Proposition 2.4 implies that $\widetilde{T}, \widetilde{\Lambda}=o_{p}(1)$. Thus, when $0<\zeta / \alpha<1$ and $\beta>1$ we have

$$
\frac{T}{\widehat{T}}=1+o_{p}(1) \quad \text { and } \quad \frac{\Lambda}{\widehat{\Lambda}}=1+o_{p}(1)
$$

Now, the first part of the theorem follows from (2.4). The value of $L_{\infty}(\beta, \zeta, \alpha)$ will be deduced in Section 6.1.

For $\zeta / \alpha \geq 1$ and $\beta>1$, the statement of the theorem follows from the fact that $\widehat{T}+\widetilde{T}=o_{p}(\mathbb{E}(\widehat{\Lambda}))(\mathrm{cf} .(2.4)$ and Proposition 2.4) together with the fact that $\widehat{\Lambda}=\mathbb{E}(\widehat{\Lambda})(1+$ $\left.o_{p}(1)\right)$ (cf. Proposition 2.3). This argument also works for $\beta \leq 1$.

\section{PRELIMINARY RESULTS}

For two vertices $u, v$ of types $t_{u}$ and $t_{v}$, respectively, we define

$$
A_{u, v}:=\frac{N}{v} e^{-\frac{\zeta}{2}\left(t_{u}+t_{v}\right)}=e^{-\frac{\zeta}{2}\left(R-t_{u}-t_{v}\right)} .
$$

For two vertices $u, v$ we write $u \sim v$ to indicate that $u$ is adjacent to $v$. The following lemma is a special case of Lemma 2.4 in [17]. 
Lemma 3.1. Let $\beta>0$. There exists a constant $C_{\beta}>0$ such that, uniformly for all distinct pairs $u, v \in \mathrm{V}_{N}$ such that $t_{u}+t_{v}<R-2 \omega(N)$, we have

$$
\mathbb{P}\left(u \sim v \mid t_{u}, t_{v}\right)= \begin{cases}(1+o(1)) \frac{C_{\beta}}{A_{u, v}}, & \text { if } \beta>1 \\ (1+o(1)) \frac{C_{\beta} \ln A_{u, v}}{A_{u, v}}, & \text { if } \beta=1 . \\ (1+o(1)) \frac{C_{\beta}}{A_{u, v}^{\beta}}, & \text { if } \beta<1 .\end{cases}
$$

In particular,

$$
C_{\beta}= \begin{cases}\frac{2}{\beta} \sin ^{-1}\left(\frac{\pi}{\beta}\right), & \text { if } \beta>1 \\ \frac{2}{\pi}, & \text { if } \beta=1 \\ \frac{1}{\sqrt{\pi}} \frac{\Gamma\left(\frac{1-\beta}{2}\right)}{\Gamma\left(1-\frac{\beta}{2}\right)}, & \text { if } \beta<1\end{cases}
$$

We will need the following fact, which expresses the hyperbolic distance between two points of given types as a function of their relative angle. For two points $u$ and $v$ in $\mathcal{D}_{R}$, we denote by $\theta_{u, v} \in[0, \pi]$ the relative angle between $u$ and $v$ (with the center of $\mathcal{D}_{R}$ being the reference point).

Lemma 3.2. Let $u, v \in \mathcal{D}_{R}$ be two distinct points of types $t_{u}$ and $t_{v}$, respectively. Moreover, set

$$
\bar{\theta}_{u, v}:=\left(e^{-2 \zeta\left(R-t_{u}\right)}+e^{-2 \zeta\left(R-t_{v}\right)}\right)^{1 / 2}=v^{2}\left\{\frac{e^{2 \zeta t_{u}}}{N^{4}}+\frac{e^{2 \zeta t_{v}}}{N^{4}}\right\}^{1 / 2}
$$

and assume that $\theta_{u, v} \gg \bar{\theta}_{u, v}$. Then

$$
p_{u, v}=\frac{1}{C A_{u, v}^{\beta} \sin ^{\beta}\left(\theta_{u, v} / 2\right)+1},
$$

where $C=1+o(1)$, uniformly for any $t_{u}, t_{v}<R / 2-\omega(N)$.

Proof. We will use the following fact, which relates the hyperbolic distance between two points of given types with their relative angle.

Fact 3.3. Lemma 2.3, [17]. For every $\beta>0$ and $0<\zeta / \alpha<2$, let $u$, $v$ be two distinct points of $\mathcal{D}_{R}$. If $\bar{\theta}_{u, v} \ll \theta_{u, v} \leq \pi$, then

$$
d(u, v)=2 R-\left(t_{u}+t_{v}\right)+\frac{2}{\zeta} \log \sin \left(\frac{\theta_{u, v}}{2}\right)+\Theta\left(\left(\frac{\bar{\theta}_{u, v}}{\theta_{u, v}}\right)^{2}\right),
$$

uniformly for all $u, v$ with $t_{u}, t_{v}<R / 2-\omega(N)$.

Hence, whenever $\theta_{u, v} \gg \bar{\theta}_{u, v}$ we have

$$
e^{\beta \frac{\zeta}{2}(d(u, v)-R)}=C e^{\beta \frac{\zeta}{2}\left(R-\left(t_{u}+t_{v}\right)\right)} e^{\beta \log \sin \left(\theta_{u, v} / 2\right)},
$$


where $C=1+o(1)$, uniformly for any $t_{u}, t_{v}<R / 2-\omega(N)$; note that the error term depends on $u$ and $v$ but is uniformly bounded by a function that is $o(1)$. The lemma follows from the definition of $p_{u, v}$ together with (3.1).

Finally, note that the density function of the type of a vertex $u$ is

$$
\bar{\rho}_{N}\left(t_{u}\right):=\alpha \frac{\sinh \left(\alpha\left(R-t_{u}\right)\right)}{\cosh (\alpha R)-1} .
$$

We will be using this density quite frequently, because we will often be conditioning on the types of the vertices under consideration. It is not difficult to see that this density can be approximated by an exponential density.

Claim 3.4. For any vertex $u \in \mathcal{D}_{R}$, uniformly for $t_{u}<R$ we have

$$
\bar{\rho}_{N}\left(t_{u}\right) \leq(1+o(1)) \alpha e^{-\alpha t_{u}} .
$$

Moreover, uniformly for all $0<t_{u} \leq \lambda R$, where $0<\lambda<1$, we have

$$
\bar{\rho}_{N}\left(t_{u}\right)=(1+o(1)) \alpha e^{-\alpha t_{u}} .
$$

Proof. Starting from the definition we get:

$$
\begin{aligned}
\bar{\rho}_{N}\left(t_{u}\right) & =\alpha \frac{\sinh \left(\alpha\left(R-t_{u}\right)\right)}{\cosh (\alpha R)-1} \leq 2 \alpha \frac{\sinh \left(\alpha\left(R-t_{u}\right)\right)}{e^{\alpha R}}(1+o(1)) \\
& =\alpha \frac{e^{\alpha\left(R-t_{u}\right)}}{e^{\alpha R}}(1+o(1))=\alpha e^{-\alpha t_{u}}(1+o(1)) .
\end{aligned}
$$

The lower bound under the condition $t_{u} \leq \lambda R$ can be proven by arguing as follows:

$$
\sinh \left(\alpha\left(R-t_{u}\right)\right)=\cosh \left(\alpha\left(R-t_{u}\right)\right)-e^{-\alpha\left(R-t_{u}\right)}=\cosh \left(\alpha\left(R-t_{u}\right)\right)(1-o(1)),
$$

uniformly over $t_{u} \leq \lambda R$.

Furthermore, we have:

$$
\frac{\cosh \left(\alpha\left(R-t_{u}\right)\right)}{\cosh (\alpha R)-1} \geq \frac{\cosh \left(\alpha\left(R-t_{u}\right)\right)}{\cosh (\alpha R)}(1+o(1)) .
$$

Hence,

$$
\begin{aligned}
\bar{\rho}_{N}\left(t_{u}\right) & =\alpha \frac{\sinh \left(\alpha\left(R-t_{u}\right)\right)}{\cosh (\alpha R)-1} \geq \alpha \frac{\cosh \left(\alpha\left(R-t_{u}\right)\right)}{\cosh (\alpha R)}(1+o(1)) \\
& \geq \alpha \frac{e^{\alpha\left(R-t_{u}\right)}}{e^{\alpha R}}(1+o(1))=\alpha e^{-\alpha t_{u}}(1+o(1)) .
\end{aligned}
$$

At this point the statement follows.

Now, Claim 3.4, together with the fact that $N \asymp e^{\zeta R / 2}$, implies the following. 
Corollary 3.5. For any $\zeta, \alpha>0$, conditional on $u \in \mathrm{V}_{N}$, we have

$$
\mathbb{P}\left(t_{u} \leq \frac{\zeta}{2 \alpha} R+\omega(N)\right)=1-o\left(N^{-1}\right) .
$$

In particular, a.a.s. for all $u \in \mathrm{V}_{N}$

$$
t_{u} \leq \frac{\zeta}{2 \alpha} R+\omega(N)
$$

\section{ON INCOMPLETE TRIANGLES: PROOF OF PROPOSITION 2.1}

In this section, we calculate the expected number of incomplete triangles with all their vertices having type less than $R / 2-\omega(N)$, that is, being typical vertices. In particular, we give the proofs of (2.1), (2.2), and (2.3).

Let us introduce the following notation: for every triple of vertices $u, v, w$, such that $t_{u}, t_{v}, t_{w}<R / 2-\omega(N)$ (being $\omega(N)$, an arbitrary, slowly growing function), we denote by $\Lambda(u, v ; w)$ the event that the triple $u, v, w$ forms an incomplete triangle pivoted at $w$. In other words, $u, v$, and $w$ form a path of length 2 with $w$ being the middle vertex. Note that there are exactly $3\left(\begin{array}{c}N \\ 3\end{array}\right)$ such choices.

Similarly, for every triple of vertices $u, v, w$ such that $t_{u}, t_{v}, t_{w}<R / 2-\omega(N)$, we denote by $\Delta(u, v, w)$ the event that the triple $u, v, w$ forms a triangle. At this point, we introduce the following parameters, which will be used throughout the paper:

$$
\beta^{\prime}:= \begin{cases}1, & \text { if } \beta \geq 1 \\ \beta, & \text { if } \beta<1\end{cases}
$$

as well as

$$
\delta=\delta(\beta, 1):=\left\{\begin{array}{ll}
1 & \text { if } \beta=1 \\
0 & \text { otherwise }
\end{array} .\right.
$$

We start from a simple observation: conditional on the types of $u, v, w$, the presence of the edges $\{u w\}$ and $\{v w\}$ are independent events. Thereby, we have

$$
\mathbb{P}\left(\Lambda(u, v ; w) \mid t_{u}, t_{v}, t_{w}\right)=\mathbb{P}\left(u \sim w \mid t_{u}, t_{w}\right) \mathbb{P}\left(v \sim w \mid t_{v}, t_{w}\right) .
$$

This implies:

$$
\begin{aligned}
\mathbb{P}\left(\Lambda(u, v ; w) \mid t_{u}, t_{v}, t_{w}\right) & \asymp \frac{\left(\ln \left(A_{u, w}\right) \ln \left(A_{v, w}\right)\right)^{\delta}}{A_{u, w}^{\beta^{\prime}} A_{v, w}^{\beta^{\prime}}} \\
& =\frac{\left(\ln \left(A_{u, w}\right) \ln \left(A_{v, w}\right)\right)^{\delta} e^{\beta^{\prime} \zeta t_{w}+\left(\beta^{\prime} \zeta / 2\right)\left(t_{u}+t_{v}\right)}}{N^{2 \beta^{\prime}}} .
\end{aligned}
$$

Hence, to determine the expected value of $\widehat{\Lambda}$, we need to integrate these expressions with respect to $t_{u}, t_{v}, t_{w}$ (using the density given by Claim 3.4) and subsequently multiply the outcome by $3\left(\begin{array}{c}N \\ 3\end{array}\right)$. 
More precisely, we have

$$
\begin{aligned}
\mathbb{P}(\Lambda(u, v ; w)) \asymp & N^{-2 \beta^{\prime}} \int_{0}^{R / 2-\omega(N)} \int_{0}^{R / 2-\omega(N)} e^{\left(\beta^{\prime} \zeta / 2-\alpha\right) t_{u}} e^{\left(\beta^{\prime} \zeta / 2-\alpha\right) t_{v}} \\
& \times\left(\int_{0}^{R / 2-\omega(N)}\left(R-t_{u}-t_{w}\right)^{\delta}\left(R-t_{v}-t_{w}\right)^{\delta} e^{\left(\beta^{\prime} \zeta-\alpha\right) t_{w}} d t_{w}\right) d t_{u} d t_{v} .
\end{aligned}
$$

Now, observe that a lower bound for the above integral can be obtained by reducing the domain of integration of $t_{u}$ and $t_{v}$ up to $R / 4$. Thus, if $t_{u}, t_{v} \leq R / 4$, we have $\left(R-t_{u}-\right.$ $\left.t_{w}\right)^{\delta}\left(R-t_{v}-t_{w}\right)^{\delta} \geq\left(3 R / 4-t_{w}\right)^{2 \delta}$. This simplifies the calculations considerably, yielding:

$$
\begin{aligned}
\mathbb{P}(\Lambda(u, v ; w)) & \gtrsim N^{-2 \beta^{\prime}}\left(\int_{0}^{R / 4} e^{\left(\beta^{\prime} \zeta / 2-\alpha\right) t_{u}} d t_{u}\right)^{2} \int_{0}^{R / 2-\omega(N)}\left(3 R / 4-t_{w}\right)^{2 \delta} e^{\left(\beta^{\prime} \zeta-\alpha\right) t_{w}} d t_{w} \\
& \gtrsim R^{2 \delta} N^{-2 \beta^{\prime}} \int_{0}^{R / 2-\omega(N)} e^{\left(\beta^{\prime} \zeta-\alpha\right) t_{w}} d t_{w} .
\end{aligned}
$$

We obtain an upper bound using $\left(R-t_{u}-t_{w}\right)^{\delta}\left(R-t_{v}-t_{w}\right)^{\delta}<\left(R-t_{w}\right)^{2 \delta}$. This yields

$$
\begin{aligned}
\mathbb{P}(\Lambda(u, v ; w)) & \lesssim N^{-2 \beta^{\prime}}\left(\int_{0}^{R / 2-\omega(N)} e^{\left(\beta^{\prime} \zeta / 2-\alpha\right) t_{u}} d t_{u}\right)^{2} \int_{0}^{R / 2-\omega(N)}\left(R-t_{w}\right)^{2 \delta} e^{\left(\beta^{\prime} \zeta-\alpha\right) t_{w}} d t_{w} \\
& \lesssim R^{2 \delta} N^{-2 \beta^{\prime}} \int_{0}^{R / 2-\omega(N)} e^{\left(\beta^{\prime} \zeta-\alpha\right) t_{w}} d t_{w}
\end{aligned}
$$

At this point, we need a case distinction according to the value of $\beta^{\prime} \zeta / \alpha$, leading to

$$
\mathbb{P}(\Lambda(u, v ; w)) \asymp\left\{\begin{array}{ll}
R^{2 \delta} N^{-2 \beta^{\prime}}, & \text { if } \beta^{\prime} \zeta / \alpha<1 \\
R^{1+2 \delta} N^{-2 \beta^{\prime}}, & \text { if } \beta^{\prime} \zeta / \alpha=1 . \\
R^{2 \delta} N^{-\beta^{\prime}-\alpha / \zeta} e^{-\left(\beta^{\prime} \zeta-\alpha\right) \omega(N)}, & \text { if } \beta^{\prime} \zeta / \alpha>1
\end{array} .\right.
$$

Multiplying these expressions by $3\left(\begin{array}{c}N \\ 3\end{array}\right)$, we deduce the statement for $\mathbb{E}(\widehat{\Lambda})$.

\section{PROOF OF PROPOSITION 2.3}

In this section, we show the concentration of the random variables $\widehat{\Lambda}$ and $\widehat{T}$ around $\mathbb{E}(\widehat{\Lambda})$ and $\mathbb{E}(\widehat{T})$, respectively. We will do so by bounding their second moment. For every triple of distinct vertices $u, v, w$, recall that $\Lambda(u, v ; w)$ denotes the event that the vertices $u, v, w$ form an incomplete triangle, with $w$ being the pivoting vertex. For a triple of distinct vertices $u, v$ and $w$ we denote by $\{u, v ; w\}$ the set $\{\{u, v\}, w\}$. We have

$$
\begin{aligned}
\mathbb{E}\left(\widehat{\Lambda}^{2}\right) & =\mathbb{E}\left[\left(\sum_{\{u, v ; w\}} \mathbf{1}_{\{\Lambda(u, v ; w)\}}\right)^{2}\right] \\
& =\sum_{\{u, v ; w\}} \mathbb{E}\left(\mathbf{1}_{\{\Lambda(u, v ; w)\}}^{2}\right)+\sum_{\left\{u_{2}, v_{2} ; w_{2}\right\} \neq\left\{u_{1}, v_{1} ; w_{1}\right\}} \mathbb{E}\left(\mathbf{1}_{\left\{\Lambda\left(u_{1}, v_{1} ; w_{1}\right)\right\}} \mathbf{1}_{\left\{\Lambda\left(u_{2}, v_{2} ; w_{2}\right)\right\}}\right) \\
& =\mathbb{E}(\widehat{\Lambda})+\sum_{\left\{u_{2}, v_{2} ; w_{2}\right\} \neq\left\{u_{1}, v_{1} ; w_{1}\right\}} \mathbb{E}\left(\mathbf{1}_{\left\{\Lambda\left(u_{1}, v_{1} ; w_{1}\right)\right\}} \mathbf{1}_{\left\{\Lambda\left(u_{2}, v_{2} ; w_{2}\right)\right\}}\right),
\end{aligned}
$$

where the double sum is taken over pairs of distinct triples of vertices. 
Similarly, with $\{u, v, w\}$ denoting a set of pairwise distinct vertices, we write

$$
\mathbb{E}\left(\widehat{T}^{2}\right)=\mathbb{E}(\widehat{T})+\sum_{\left\{u_{1}, v_{1}, w_{1}\right\} \neq\left\{u_{2}, v_{2}, w_{2}\right\}} \mathbb{E}\left(\mathbf{1}_{\left\{\Delta\left(u_{1}, v_{1}, w_{1}\right)\right\}} \mathbf{1}_{\left\{\Delta\left(u_{2}, v_{2}, w_{2}\right)\right\}}\right) .
$$

In order to show concentration of $\widehat{\Lambda}$, we will show the following statement.

Lemma 5.1. For any $0<\frac{\zeta}{\alpha}<2$ and $\beta>0$, we have

$$
\mathbb{E}\left(\widehat{\Lambda}^{2}\right)=\mathbb{E}^{2}(\widehat{\Lambda})(1+o(1))
$$

Proof. To evaluate the second moment of $\widehat{\Lambda}$, we need to control the dependencies between every two triples of vertices $u_{1}, v_{1}, w_{1}$ and $u_{2}, v_{2}, w_{2}$, assuming that the vertices $w_{1}$ and $w_{2}$ are the pivoting vertices. More precisely, we have eight possible configurations:

1. $\left\{u_{1}, v_{1}, w_{1}\right\} \cap\left\{u_{2}, v_{2}, w_{2}\right\}=\emptyset$;

2. $u_{1}=u_{2}$ with $\left\{v_{1}, w_{1}\right\} \cap\left\{v_{2}, w_{2}\right\}=\emptyset$ (or, analogously, $v_{1}=v_{2}$ and $\left\{u_{1}, w_{1}\right\} \cap\left\{u_{2}, w_{2}\right\}=$ $\emptyset)$;

3. $w_{1}=w_{2}$ and $\left\{u_{1}, v_{1}\right\} \cap\left\{u_{2}, v_{2}\right\}=\emptyset$;

4. $u_{1}=u_{2}, v_{1}=v_{2}, w_{1} \neq w_{2}$;

5. $w_{1}=u_{2}, v_{1}=w_{2}$ and $v_{1} \neq u_{1}$;

6. $w_{1}=w_{2}, u_{1}=u_{2}, v_{1} \neq v_{2}\left(\right.$ or $\left.w_{1}=w_{2}, v_{1}=v_{2}, u_{1} \neq u_{2}\right)$.

7. $v_{1}=w_{2}$ and $\left\{u_{1}, w_{1}\right\} \cap\left\{u_{2}, v_{2}\right\}=\emptyset$.

8. $w_{1}=u_{2}, v_{1}=v_{2}$ and $u_{1} \neq w_{2}$.

We denote by $\widehat{\Lambda}_{i}$ the contribution in (5.1) of the terms that correspond to case $i$ for $i=1, \ldots, 8$. Our aim is to show that

$$
\widehat{\Lambda}_{1}=\mathbb{E}^{2}(\widehat{\Lambda})\left(1+o_{p}(1)\right),
$$

and for each $i=2, \ldots, 8$ we have

$$
\widehat{\Lambda}_{i}=o_{p}\left(\mathbb{E}^{2}(\widehat{\Lambda})\right) .
$$

Note that Proposition 2.1 implies that

$$
\mathbb{E}(\widehat{\Lambda}) \gtrsim\left\{\begin{array}{ll}
N^{3-2 \beta^{\prime}}, & \text { if } \beta^{\prime} \zeta / \alpha \leq 1 \\
N^{3-\beta^{\prime}-\alpha / \zeta} e^{-\left(\beta^{\prime} \zeta-\alpha\right) \omega(N)}, & \text { if } \beta^{\prime} \zeta / \alpha>1
\end{array},\right.
$$

where $\beta^{\prime}$ is defined in (4.1). To deduce (5.3), we will compare the polynomial terms that bound $\widehat{\Lambda}_{i}$ with the polynomial terms in (5.4): hence, from now on we consider only the polynomial terms in $N$.

Now, the probability that the event described in Cases 4 and 8 occurs can be bounded from above by the probability of having a path of length three. Hence, all events listed in Cases 2-8 can be reduced to the event of certain trees appearing in the system. More precisely, the probability of any of the events of Cases 2-8 is bounded from above by the probability of a certain tree with three (resp. four) edges and four (resp. five) vertices to be present in the graph. In general, we obtain the following result. 
Claim 5.2. Let $\mathcal{T}_{k}$ be a tree on $k \geq 2$ vertices $u_{1}, u_{2}, \ldots, u_{k}$ that have degrees $n_{1}, n_{2}, \ldots, n_{k}$, where $k$ is fixed. Then

$$
\begin{aligned}
& \mathbb{E}\left(\# \text { copies of } \mathcal{T}_{k} \text { in } \mathcal{G}(N ; \zeta, \alpha, \beta, \nu)\right) \\
& \quad \asymp N^{k-\beta^{\prime}(k-1)} R^{\delta(k-1)} \prod_{i=1}^{k} \max \left\{1, R, N^{\beta^{\prime} n_{i} / 2-\alpha / \zeta} e^{-\omega(N)\left(\beta^{\prime} \zeta n_{i} / 2-\alpha\right)}\right\} .
\end{aligned}
$$

Proof. We will calculate the probability that vertices $v_{1}, \ldots, v_{k}$ form a tree that is isomorphic to $\mathcal{T}_{k}$, where $v_{i}$ is identified with $u_{i}$. We shall give an estimate first on the probability that the $v_{i} \mathrm{~s}$ form this tree conditional on their types. So, suppose that the vertices $v_{1}, v_{2}, \ldots, v_{k}$ have types $t_{u_{1}}, t_{u_{2}}, \ldots, t_{u_{k}}$, respectively.

We shall expose/embed the positions of $v_{1}, v_{2}, \ldots, v_{k}$ according to a breadth-first ordering of the vertices of $\mathcal{T}_{k}$. Without loss of generality, let us assume that $v_{1}$ is the root of $\mathcal{T}_{k}$. The breadth-first search algorithm discovers in each round the children of an internal vertex, starting at $v_{1}$. If the rooted $\mathcal{T}_{k}$ has $\ell$ leaves, then there will be $k-\ell$ rounds. Also, assume for simplicity, that during round $i$, the children of vertex $v_{i}$ will be exposed, for $i=1, \ldots, k-\ell$. Let $\mathcal{T}_{k}^{(i)}$ denote the subtree of $\mathcal{T}_{k}$ that has been revealed after $i$ rounds of the breadth-first search. With a slight abuse of notation, we also use the symbol $\mathcal{T}_{k}^{(i)}$ to denote the event that the subtree $\mathcal{T}_{k}^{(i)}$ has been embedded. adjacency)

Consider $\mathcal{T}_{k}^{(1)}$ first. If $v_{1}$ has $v_{i_{1}}, \ldots, v_{i_{n_{1}}}$ as its children, then (with $\sim$ denoting vertex

$$
\begin{aligned}
& \mathbb{P}\left(\mathcal{T}_{k}^{(1)} \mid t_{v_{1}}, \ldots, t_{v_{k}}\right)=\prod_{j=1}^{n_{1}} \mathbb{P}\left(v_{i_{j}} \sim v_{1} \mid t_{v_{1}}, t_{v_{i_{j}}}\right) \\
& \stackrel{\check{L}^{\mathrm{Lemma}}}{{ }^{3.1}} N^{-\beta^{\prime} n_{1}} R^{\delta n_{1}} e^{\beta^{\prime} \zeta t_{v_{1}} n_{1} / 2} \prod_{j=1}^{n_{1}} e^{\beta^{\prime} \zeta t_{v_{j}} / 2} .
\end{aligned}
$$

Suppose that $\mathcal{T}_{k}^{(i)}$ has $e_{i}$ edges and let $V_{i}$ denote its set of vertices with $\mathcal{L}_{i} \subset V_{i}$ being its set of leaves. Assume that for $i$ that satisfies $1 \leq i<k-\ell$, we have shown that

$$
\mathbb{P}\left(\mathcal{T}_{k}^{(i)} \mid t_{v_{1}}, \ldots, t_{v_{k}}\right) \asymp N^{-\beta^{\prime} e_{i}} R^{\delta e_{i}} \prod_{v_{j} \in V_{i} \backslash \mathcal{L}_{i}} e^{\beta^{\prime} \zeta t_{v_{j}} n_{j} / 2} \prod_{v_{j} \in \mathcal{L}_{i}} e^{\beta^{\prime} \zeta t_{v_{j}} / 2} .
$$

In the above expression, the hidden constants do depend on $i$.

We shall derive a similar expression for $\mathcal{T}_{k}^{(i+1)}$. Assume that $v_{i+1}$, which has to be a leaf in $\mathcal{T}_{k}^{(i)}$, has $v_{j_{1}}, \ldots, v_{j_{n_{i+1}-1}}$ as its children that are to be exposed.

$$
\begin{aligned}
\mathbb{P}\left(\mathcal{T}_{k}^{(i+1)} \mid t_{v_{1}}, \ldots, t_{v_{k}}\right) & =\mathbb{P}\left(\left\{\cap_{s=1}^{n_{i+1}-1} v_{i+1} \sim v_{j_{s}}\right\} \cap \mathcal{T}_{k}^{(i)} \mid t_{v_{1}}, \ldots, t_{v_{k}}\right) \\
& =\mathbb{P}\left(\left\{\cap_{s=1}^{n_{i+1}-1} v_{i+1} \sim v_{j_{s}}\right\} \mid t_{v_{1}}, \ldots, t_{v_{k}}\right) \mathbb{P}\left(\mathcal{T}_{k}^{(i)} \mid t_{v_{1}}, \ldots, t_{v_{k}}\right) .
\end{aligned}
$$

The first term on the right-hand side can be calculated as in (5.5):

$$
\mathbb{P}\left(\left\{\cap_{s=1}^{n_{i+1}-1} v_{i+1} \sim v_{j_{s}}\right\} \mid t_{v_{1}}, \ldots, t_{v_{k}}\right)=N^{-\beta^{\prime}\left(n_{i+1}-1\right)} R^{\delta\left(n_{i+1}-1\right)} e^{\beta^{\prime} \zeta t_{v_{i+1}}\left(n_{i+1}-1\right) / 2} \prod_{s=1}^{n_{i+1}-1} e^{\beta^{\prime} \zeta t_{v_{s}} / 2} .
$$


The second term is given by (5.6). Now, observe that $\mathcal{L}_{i+1}=\mathcal{L}_{i} \cup\left\{v_{j_{1}}, \ldots, v_{j_{n_{i+1}}-1}\right\} \backslash v_{i+1}$ and $v_{i+1} \in V_{i+1} \backslash \mathcal{L}_{i+1}$. Also, $e_{i+1}=e_{i}+\left(n_{i+1}-1\right)$. Therefore,

$$
\begin{aligned}
\mathbb{P}\left(\mathcal{T}_{k}^{(i+1)} \mid t_{v_{1}}, \ldots, t_{v_{k}}\right) \asymp & \left(N^{-\beta^{\prime}\left(n_{i+1}-1\right)} R^{\delta\left(n_{i+1}-1\right)} e^{\beta^{\prime} \zeta t_{v_{i+1}}\left(n_{i+1}-1\right) / 2} \prod_{s=1}^{n_{i+1}-1} e^{\beta^{\prime} \zeta t_{v_{j}} / 2}\right) \\
& \times\left(N^{-\beta^{\prime} e_{i}} R^{\delta e_{i}} \prod_{v_{j} \in V_{i} \backslash \mathcal{L}_{i}} e^{\beta^{\prime} \zeta t_{v_{j}} n_{j} / 2} \prod_{v_{j} \in \mathcal{L}_{i}} e^{\beta^{\prime} \zeta t_{v_{j}} / 2}\right) \\
= & N^{-\beta^{\prime}\left(e_{i}+n_{i+1}-1\right)} R^{\delta\left(e_{i}+n_{i+1}-1\right)} \prod_{v_{j} \in V_{i+1} \backslash \mathcal{L}_{i+1}} e^{\beta^{\prime} \zeta t_{v_{j}} n_{j} / 2} \prod_{v_{j} \in \mathcal{L}_{i+1}} e^{\beta^{\prime} \zeta t_{v_{j}} / 2} \\
= & N^{-\beta^{\prime} e_{i+1}} R^{\delta e_{i+1}} \prod_{v_{j} \in V_{i+1} \backslash \mathcal{L}_{i+1}} e^{\beta^{\prime} \zeta t_{v_{j}} n_{j} / 2} \prod_{v_{j} \in \mathcal{L}_{i+1}} e^{\beta^{\prime} \zeta t_{v_{j}} / 2} .
\end{aligned}
$$

Thus,

$$
\begin{gathered}
\mathbb{P}\left(\mathcal{T}_{k}^{(k-\ell)} \mid t_{v_{1}}, \ldots, t_{v_{k}}\right) \asymp N^{-\beta^{\prime} e_{k-\ell}} R^{\delta e_{k-\ell}} \prod_{v_{j} \in V_{i} \backslash \mathcal{L}_{k-\ell}} e^{\beta^{\prime} \zeta t_{v_{j}} n_{j} / 2} \prod_{v_{j} \in \mathcal{L}_{k-\ell}} e^{\beta^{\prime} \zeta t_{v_{i}} / 2} \\
\stackrel{\mathcal{T}_{k}^{(k-\ell)}=\mathcal{T}_{k}}{=} N^{-\beta^{\prime}(k-1)} R^{\delta(k-1)} \prod_{i=1}^{k} e^{\beta^{\prime} \zeta n_{i} t_{v_{i}} / 2} .
\end{gathered}
$$

Integrating over the types, we get

$\mathbb{P}\left(v_{1}, v_{2}, \ldots, v_{k}\right.$ form an embedding of $\left.\mathcal{T}_{k}\right)$

$$
\begin{aligned}
& \asymp\left(\frac{R^{\delta}}{N^{\beta^{\prime}}}\right)^{k-1} \int_{0}^{R / 2-\omega(N)} \cdots \int_{0}^{R / 2-\omega(N)} e^{\beta^{\prime} \zeta n_{1} t_{u_{1}} / 2-\alpha t_{u_{1}}} \cdots e^{\beta^{\prime} \zeta n_{k} t_{u_{k}} / 2-\alpha t_{u_{k}}} d t_{u_{1}} \cdots d t_{u_{k}} \\
& \asymp N^{-\beta^{\prime}(k-1)} R^{\delta(k-1)} \prod_{i=1}^{k} \max \left\{1, R, e^{(R / 2-\omega(N))\left(\beta^{\prime} \zeta n_{i} / 2-\alpha\right)}\right\} .
\end{aligned}
$$

The number of choices for the vertices $v_{1}, \ldots, v_{k}$ and their arrangement into a copy of $\mathcal{T}_{k}$ is proportional to $N^{k}$. Hence, multiplying the above expression by $N^{k}$ yields the statement of the claim.

Now, suppose that there are exactly $\ell \geq 1$ vertices with degrees larger than $2 \alpha /\left(\beta^{\prime} \zeta\right)$. Without loss of generality, we can re-label the vertices in such a way that $u_{1}, u_{2}, \ldots, u_{\ell}$ are such that $n_{i}>2 \alpha /\left(\beta^{\prime} \zeta\right)$ for $i \in\{1,2, \ldots, \ell\}$. Furthermore, assume that there are $\ell^{\prime}$ vertices with $n_{i}=2 \alpha /\left(\beta^{\prime} \zeta\right)$. This implies that

$$
\prod_{i=1}^{k} \max \left\{1, R, e^{(R / 2-\omega(N))\left(\beta^{\prime} \zeta n_{i} / 2-\alpha\right)}\right\}=R^{\ell^{\prime}} e^{(R / 2-\omega(N))\left(\beta^{\prime} \zeta\left(\sum_{j=1}^{\ell} n_{j}\right) / 2-\ell \alpha\right)} .
$$

Because the $n_{j}$ s are degrees of vertices in $\mathcal{T}_{k}$, we have $\sum_{j=1}^{\ell} n_{j} \leq 2(k-1)$, because there are $k-1$ edges in $\mathcal{T}_{k}$. 
Thus,

$$
\beta^{\prime} \zeta\left(\sum_{j=1}^{\ell} n_{j}\right) / 2-\ell \alpha \leq \beta^{\prime} \zeta 2(k-1) / 2-\ell \alpha=\beta^{\prime} \zeta(k-1)-\ell \alpha \stackrel{\ell \geq 1}{\leq} \beta^{\prime} \zeta(k-1)-\alpha .
$$

The last quantity corresponds to the case when $\mathcal{T}_{k}$ is a star; in fact, this is the case when $\ell=1$ and $n_{1}=k-1$. (Note that even if $\ell=0$, then the star provides an upper bound.) This implies that for $i=2, \ldots, 8$ we have $\mathbb{E}\left(\widehat{\Lambda}_{i}\right)=o\left(\max \left\{\mathbb{E}\left(\widehat{\Lambda}_{3}\right), \mathbb{E}\left(\widehat{\Lambda}_{6}\right)\right\}\right)$. So, to show (5.3), it suffices to prove it for Cases 3 and 6. However, we begin Case 1, which shows (5.2).

Case 1: the two incomplete triangles are clearly independent, since they are disjoint. In other words, the realization of the event $\left\{u_{1} \sim w_{1} \sim v_{1}\right\}$ gives no information about the triple $\left\{u_{2}, v_{2}, w_{2}\right\}$. Thus,

$$
\mathbb{P}\left(\Lambda\left(u_{1}, v_{1} ; w_{1}\right) \cap \Lambda\left(u_{2}, v_{2} ; w_{2}\right)\right)=\mathbb{P}\left(\Lambda\left(u_{1}, v_{1} ; w_{1}\right)\right) \mathbb{P}\left(\Lambda\left(u_{2}, v_{2} ; w_{2}\right)\right) .
$$

There are $3\left(\begin{array}{c}N \\ 3\end{array}\right)$ ways to select three distinct vertices $u_{1}, v_{1}, w_{1}$ and distinguish among them the central vertex of the path. Having chosen those, there are $3\left(\begin{array}{c}N-3 \\ 3\end{array}\right)$ ways for selecting the second triple. Therefore,

$$
\mathbb{E}\left(\widehat{\Lambda}_{1}\right)=9\left(\begin{array}{c}
N \\
3
\end{array}\right)\left(\begin{array}{c}
N-3 \\
3
\end{array}\right) \mathbb{P}\left(\Lambda\left(u_{1}, v_{1} ; w_{1}\right)\right) \mathbb{P}\left(\Lambda\left(u_{2}, v_{2} ; w_{2}\right)\right)=\mathbb{E}^{2}(\widehat{\Lambda})(1-o(1)),
$$

which is (5.2).

Case 3: By Claim 5.2, the contribution of Case 3 to $\mathbb{E}\left(\widehat{\Lambda}^{2}\right)$ is

$$
\mathbb{E}\left(\widehat{\Lambda}_{3}\right) \lesssim \begin{cases}N^{5} \frac{R^{4 \delta}}{N^{4 \beta^{\prime}}} \asymp R^{4 \delta} N^{5-4 \beta^{\prime}}, & \text { if } \frac{\beta^{\prime} \zeta}{\alpha}<\frac{1}{2} \\ N^{5} \frac{R^{4 \delta+1}}{N^{4 \beta^{\prime}}} \asymp N^{5-4 \beta^{\prime}} R^{4 \delta+1}, & \text { if } \frac{\beta^{\prime} \zeta}{\alpha}=\frac{1}{2} \\ R^{4 \delta} N^{5} \frac{N^{2 \beta^{\prime}-\alpha / \zeta} e^{-\left(2 \beta^{\prime} \zeta-\alpha\right) \omega(N)}}{N^{4 \beta^{\prime}}}, & \text { if } \frac{\beta^{\prime} \zeta}{\alpha}>\frac{1}{2}\end{cases}
$$

Now it suffices to take into account the exponents of the terms in $N$.

When $\beta^{\prime} \zeta / \alpha \leq 1 / 2$, then (5.3) holds since $5-4 \beta^{\prime}<2\left(3-2 \beta^{\prime}\right)$. When $\beta^{\prime} \zeta / \alpha>1 / 2$, we need to have

$$
5-2 \beta^{\prime}-\alpha / \zeta \leq 2\left(3-2 \beta^{\prime}\right), \text { if } 1 / 2<\beta^{\prime} \zeta / \alpha \leq 1 .
$$

This suffices in order to show (5.3) because of the $e^{-\left(2 \beta^{\prime} \zeta-\alpha\right) \omega(N)}$ factor. This is equivalent to $2 \beta^{\prime} \leq \alpha / \zeta+1$, which holds since $\beta^{\prime} \leq \alpha / \zeta$ and $\beta^{\prime} \leq 1$. Also, we need to have

$$
5-2 \beta^{\prime}-\alpha / \zeta<2\left(3-\beta^{\prime}-\alpha / \zeta\right) \text {, if } 1<\beta^{\prime} \zeta / \alpha .
$$

But this is equivalent to $\alpha / \zeta<1$, which also holds since $\alpha / \zeta<\beta^{\prime} \leq 1$. 
Case 6: Claim 5.2 yields:

$$
\mathbb{E}\left(\widehat{\Lambda}_{6}\right) \asymp\left\{\begin{array}{lr}
N^{4} \frac{R^{3 \delta}}{N^{3 \beta^{\prime}}}, & \text { if } \frac{\beta^{\prime} \zeta}{\alpha}<\frac{2}{3} \\
N^{4} \frac{R^{3 \delta+1}}{N^{3 \beta^{\prime}}}, & \text { if } \frac{\beta^{\prime} \zeta}{\alpha}=\frac{2}{3} \\
N^{4} \frac{R^{3 \delta} N^{\left(3 \beta^{\prime} / 2-\alpha / \zeta\right)} e^{-\left(3 \zeta \beta^{\prime} / 2-\alpha\right) \omega(N)}}{N^{3 \beta^{\prime}}}= & \\
=R^{3 \delta} N^{4-3 \beta^{\prime} / 2-\alpha / \zeta} e^{-\left(3 \beta^{\prime} \zeta / 2-\alpha\right) \omega(N)}, & \text { if } \frac{\beta^{\prime} \zeta}{\alpha}>\frac{2}{3}
\end{array} .\right.
$$

To verify (5.3) when $\beta^{\prime} \zeta / \alpha \leq 2 / 3$, note that $4-3 \beta^{\prime}<2\left(3-2 \beta^{\prime}\right.$ ) (which is equivalent to $\beta^{\prime}<2$ ) holds. When $2 / 3<\beta^{\prime} \zeta / \alpha \leq 1$, it suffices to verify that

$$
4-3 \beta^{\prime} / 2-\alpha / \zeta<2\left(3-2 \beta^{\prime}\right),
$$

which is equivalent to $5 \beta^{\prime} / 2-\alpha / \zeta<2$. But $\alpha / \zeta \geq \beta^{\prime}$, which implies that

$$
\frac{5 \beta^{\prime}}{2}-\frac{\alpha}{\zeta} \leq \frac{3 \beta^{\prime}}{2} \leq \frac{3}{2}<2
$$

Finally, assume that $\beta^{\prime} \zeta / \alpha>1$. Here, it suffices to show that

$$
4-3 \beta^{\prime} / 2-\alpha / \zeta<2\left(3-\beta^{\prime}-\alpha / \zeta\right),
$$

which is equivalent to

$$
\frac{\beta^{\prime}}{2}+\frac{\alpha}{\zeta}<2
$$

But $\alpha / \zeta<\beta^{\prime}$, whereby the left-hand side is at most $3 \beta^{\prime} / 2 \leq 3 / 2<2$. Hence, (5.3) holds also in this case.

Now we have that $\operatorname{Var}(\widehat{\Lambda})=o\left(\mathbb{E}^{2} \widehat{\Lambda}\right)$ and, therefore, the concentration of $\widehat{\Lambda}$ follows immediately from Chebyshev's inequality together with the fact that $\mathbb{E}(\widehat{\Lambda}) \rightarrow \infty$ as $N \rightarrow$ $\infty$ (cf. (5.4)).

Next we show the analogue of Lemma 5.1 for $\widehat{T}$.

Lemma 5.3. For any $0<\frac{\zeta}{\alpha}<1$ and $\beta>1$, we have

$$
\mathbb{E}\left(\widehat{T}^{2}\right)=\mathbb{E}^{2} \widehat{T}(1+o(1))
$$

Proof. The proof of this fact is almost identical to that of Lemma 5.1, which we apply to a version of the random variable $\widehat{T}$. More specifically, we let $\widehat{T}_{r}$ denote the number of rooted typical triangles, that is, the typical triangles with one distinguished vertex, which we call the root. Note that $\widehat{T}_{r}=3 \widehat{T}$, whereby $\mathbb{E}(\widehat{T}) \asymp \mathbb{E}\left(\widehat{T}_{r}\right)$.

To estimate the second moment of $\widehat{T}_{r}$, we also need to consider all different cases that cover all possible ways of intersection of the distinct triples $u_{1}, v_{1}, w_{1}$ and $u_{2}, v_{2}, w_{2}$. We denote by $\widehat{T}_{i}$ the contribution of each case in $\mathbb{E}\left(\widehat{T}^{2}\right)$. 
For three distinct vertices $u, v, w$, we let $\Delta(u, v ; w)$ denote the event that the vertices $u, v, w$ form a triangle that is rooted at $w$. If $u_{1}, v_{1}, w_{1}$ and $u_{2}, v_{2}, w_{2}$ are two disjoint triples of vertices, that is, for Case 1 we have

$$
\mathbb{P}\left(\Delta\left(u_{1}, v_{1} ; w_{1}\right) \Delta\left(u_{2}, v_{2} ; w_{2}\right)\right)=\mathbb{P}\left(\Delta\left(u_{1}, v_{1} ; w_{1}\right)\right) \mathbb{P}\left(\Delta\left(u_{2}, v_{2} ; w_{2}\right)\right),
$$

which implies that

$$
\mathbb{E}\left(\widehat{T}_{1}\right)=\mathbb{E}^{2}(\widehat{T})(1-o(1)) .
$$

For the remaining two cases (either when the triangles share a vertex or when they share an edge) we can deduce that $\mathbb{E}\left(\widehat{T}_{i}\right) / \mathbb{E}^{2}\left(\widehat{T}_{r}\right)=o(1)$, because $\mathbb{E}\left(\widehat{T}_{i}\right) \leq \mathbb{E}\left(\widehat{\Lambda}_{i}\right)$ (as every rooted triangle is contained in an incomplete triangle whose pivoting vertex is the root of the triangle) and $\mathbb{E}\left(\widehat{T}_{r}\right) \asymp \mathbb{E}(\widehat{\Lambda})$ (cf. Propositions $2.1,2.2$ ).

Again, concentration of $\widehat{T}$ follows by applying Chebyshev's inequality together with the fact that $\mathbb{E}(\widehat{T}) \rightarrow \infty$ as $N \rightarrow \infty$ (cf. (2.4)).

\section{ON THE PROBABILITY OF TRIANGLES: PROOF OF THEOREM 1.3}

In this section, we compute the probability that three typical vertices $u, v, w$ form a triangle. In particular, we find an explicit expression for such quantity, which allows us to prove Theorem 1.3.

For any two distinct points $u$ and $v$, we denote the angle of $u$ with respect to $v$ by $\vartheta_{u, v} \in(-\pi, \pi)$; here, we assume that $v$ is the reference point and $\vartheta_{u, v}$ is positive when the straight line that joins $u$ with the center of $\mathcal{D}_{R}$ is reached, if we rotate counterclockwise the straight line that joins $v$ with the center of $\mathcal{D}_{R}$.

We will approximate $\widehat{T}$ by a random variable that counts triangles whose vertices are arranged in a certain way on $\mathcal{D}_{R}$. More specifically, for three distinct vertices $u, v$, and $w$, we let $T(u, v ; w)$ denote the indicator random variable that is equal to 1 if and only if $u, v$, and $w$ form a triangle with $0 \leq \vartheta_{u, w} \leq \pi$ and $-\pi \leq \vartheta_{v, w} \leq 0$ and $t_{u}, t_{v}, t_{w}<R / 2-\omega(N)$. We let

$$
\widehat{T}^{\prime}:=\sum_{w \in V_{N}} \sum_{\substack{(u, v) ; \\ u, v \in \mathrm{V}_{N} \backslash\{w\}}} T(u, v ; w),
$$

where the second sum ranges over the set of ordered pairs of distinct vertices in $\mathrm{V}_{N} \backslash\{w\}$. Note that, in general, $\widehat{T}^{\prime}$ is not equal to $\widehat{T}$. However,

$$
\widehat{T} \leq \widehat{T}^{\prime} \leq 3 \widehat{T} .
$$

We will work with this random variable because its analysis is somewhat easier compared to that of $\widehat{T}$ due to the fact that the indicators $T(u, v ; w)$ are associated with a certain arrangement of the vertices $u, v, w$ on $\mathcal{D}_{R}$. For any two distinct vertices $u$ and $v$, we define the following quantities:

$$
\tilde{\theta}_{u, v}:=\left\{\begin{array}{ll}
\left(\omega(N) A_{u, v}\right)^{-1} & \text { if } \beta \geq 1 \\
\omega(N) A_{u, v}^{-1} & \text { if } \beta<1
\end{array} \text { and } \quad \hat{\theta}_{u, v}:=\left\{\begin{array}{ll}
\omega(N) A_{u, v}^{-1} & \text { if } \beta>1 \\
\pi-\tilde{\theta}_{u, v} & \text { if } \beta \leq 1
\end{array} .\right.\right.
$$


Informally, these define an area such that when the (relative) angle between $u$ and $v$ is within these bounds, the probability that $u$ is adjacent to $v$ is maximized.

We split the expected value of $T(u, v ; w)$ according to the value of the angle between $u$ and $w$ and that between $v$ and $w$. Our aim is to show that the main contribution to the expected value of $T(u, v ; w)$ comes from the case when $\left|\vartheta_{u, w}\right|$ and $\left|\vartheta_{v, w}\right|$ are within the bounds $\tilde{\theta}_{u, w}, \hat{\theta}_{u, w}$ and $\tilde{\theta}_{v, w}, \hat{\theta}_{v, w}$, respectively. We have

$$
\begin{aligned}
& \mathbb{P}\left(T(u, v ; w)=1 \mid t_{u}, t_{v}, t_{w}\right) \\
& =\mathbb{E}\left(T(u, v ; w) \mathbf{1}_{\left\{\tilde{\theta}_{u, w} \leq \vartheta_{u, w} \leq \hat{\theta}_{u, w},-\tilde{\theta}_{v, w} \geq \vartheta_{v, w} \geq-\hat{\theta}_{v, w}\right\}} \mid t_{u}, t_{v}, t_{w}\right) \\
& \quad+\mathbb{E}\left(T(u, v ; w)\left(1-\mathbf{1}_{\left\{\tilde{\theta}_{u, w} \leq \vartheta_{u, w} \leq \hat{\theta}_{u, w},-\tilde{\theta}_{v, w} \geq \vartheta_{v, w} \geq-\hat{\theta}_{v, w}\right\}}\right) \mid t_{u}, t_{v}, t_{w}\right) .
\end{aligned}
$$

We set

$$
\vartheta(u, v ; w):=\mathbf{1}_{\left\{\tilde{\theta}_{u, w} \leq \vartheta_{u, w} \leq \hat{\theta}_{u, w},-\tilde{\theta}_{v, w} \geq \vartheta_{v, w} \geq-\hat{\theta}_{v, w}\right\}} .
$$

Using Lemma 3.2, we express $p_{u, v}, p_{u, w}$ and $p_{v, w}$ as

$$
\begin{gathered}
p_{u, v}=\frac{1}{C A_{u, v}^{\beta} \sin ^{\beta}\left(\theta_{u, v} / 2\right)+1}, \\
p_{u, w}=\frac{1}{C A_{u, w}^{\beta} \sin ^{\beta}\left(\theta_{u, w} / 2\right)+1}, \\
p_{v, w}=\frac{1}{C A_{v, w}^{\beta} \sin ^{\beta}\left(\theta_{v, w} / 2\right)+1},
\end{gathered}
$$

as long as $\theta_{u, v} \gg \bar{\theta}_{u, v}, \theta_{u, w} \gg \bar{\theta}_{u, w}$, and $\theta_{v, w} \gg \bar{\theta}_{v, w}$, respectively. Note that the relative angle between two points is uniformly distributed in the interval $[0, \pi]$. Moreover, if $\vartheta_{u, v} \in[-\pi, \pi]$, then $\theta_{u, v}=\left|\vartheta_{u, v}\right|$ (recall that $\theta_{u, v}$ denotes the relative angle between the points $u$ and $v$ ).

Now, we focus on the first term of the right-hand side of (6.4). In order to simplify the notation, we set

$$
C_{u, v}:=e^{\frac{\zeta}{2}\left(t_{u}+t_{v}\right)}
$$

where $u, v$ are any two elements of $\mathrm{V}_{N}$.

Lemma 6.1. Let $u, v, w \in \mathrm{V}_{N}$ be three distinct vertices. Then, uniformly for $t_{u}, t_{v}, t_{w}<$ $R / 2-\omega(N)$, the following hold:

for $\beta>1$

$$
\begin{aligned}
& \mathbb{E}\left(T(u, v ; w) \vartheta(u, v ; w) \mid t_{u}, t_{v}, t_{w}\right) \\
& \quad=\frac{(1+o(1))}{\pi^{2} A_{u, w} A_{v, w}} \int_{[0, \infty)^{2}} \frac{1}{z_{1}^{\beta}+1} \frac{1}{z_{2}^{\beta}+1} \frac{1}{\left(C_{w, v} z_{1}+C_{w, u} z_{2}\right)^{\beta}+1} d z_{1} d z_{2},
\end{aligned}
$$


for $\beta=1$

$$
\begin{aligned}
& \mathbb{E}\left(T(u, v ; w) \vartheta(u, v ; w) \mid t_{u}, t_{v}, t_{w}\right) \\
& \quad \lesssim \frac{1}{A_{u, w} A_{v, w}} \int_{1 /(2 \pi \omega(N))}^{A_{v, w} / 2} \int_{1 /(2 \pi \omega(N))}^{A_{u, w} / 2} \frac{1}{z_{1}+1} \frac{1}{z_{2}+1} \frac{1}{C_{w, v} z_{1}+C_{w, u} z_{2}+1} d z_{1} d z_{2},
\end{aligned}
$$

and for $\beta<1$ we have

$$
\begin{aligned}
& \mathbb{E}\left(T(u, v ; w) \vartheta(u, v ; w) \mid t_{u}, t_{v}, t_{w}\right) \\
& \quad \lesssim \frac{1}{A_{u, w} A_{v, w}} \int_{\omega(N) / 2 \pi}^{A_{v, w} / 2} \int_{\omega(N) / 2 \pi}^{A_{u, w} / 2} \frac{1}{z_{1}^{\beta}+1} \frac{1}{z_{2}^{\beta}+1} \frac{1}{\left(C_{w, v} z_{1}+C_{w, u} z_{2}\right)^{\beta}+1} d z_{1} d z_{2} .
\end{aligned}
$$

Proof. For all $\beta>0$, we can use the expressions in (6.6) and obtain

$$
\begin{aligned}
\mathbb{E} & \left(T(u, v ; w) \mathbf{1}_{\left\{\tilde{\theta}_{u, w} \leq \vartheta_{u, w} \leq \hat{\theta}_{u, w},-\hat{\theta}_{v, w} \leq \vartheta_{v, w} \leq-\tilde{\theta}_{v, w}\right\}} \mid t_{u}, t_{v}, t_{w}\right) \\
= & \frac{1}{4 \pi^{2}} \int_{\tilde{\theta}_{u, w}}^{\hat{\theta}_{u, w}} \int_{-\hat{\theta}_{v, w}}^{-\tilde{\theta}_{v, w}} p_{u, w} p_{v, w} p_{u, v} d \vartheta_{v, w} d \vartheta_{u, w} \\
= & \frac{1}{4 \pi^{2}} \int_{\tilde{\theta}_{u, w}}^{\hat{\theta}_{u, w}} \int_{-\hat{\theta}_{v, w}}^{-\tilde{\theta}_{v, w}} \frac{1}{C A_{u, w}^{\beta} \sin ^{\beta}\left(\theta_{u, w} / 2\right)+1} \frac{1}{C A_{v, w}^{\beta} \sin ^{\beta}\left(\theta_{v, w} / 2\right)+1} \\
& \times p_{u, v} d \vartheta_{v, w} d \vartheta_{u, w} .
\end{aligned}
$$

In this case, either the relative angle between $u$ and $v$ is $\theta_{u, w}+\theta_{v, w}$, if this sum is at most $\pi$; or, it is equal to $2 \pi-\left(\theta_{u, w}+\theta_{v, w}\right)$ (this may happen when $\left.\beta \leq 1\right)$. In this case, $\sin \left(\theta_{u, v} / 2\right)=\sin \left(\pi-\frac{\theta_{u, w}+\theta_{v, w}}{2}\right)=\sin \left(\frac{\theta_{u, w}+\theta_{v, w}}{2}\right)$. Also, by the definition of $\hat{\theta}_{u, w}$ and $\hat{\theta}_{v, w}$ for $\beta \leq 1$, it follows that $\theta_{u, v} \geq \hat{\theta}_{u, w}+\hat{\theta}_{v, w}$. (Note that this issue does not come up when $\beta>1$; for $N$ sufficiently large, $\theta_{u, w}+\theta_{v, w}$ is much smaller than $\pi$.)

Thus, in order to use the expression in (6.6) for $p_{u, v}$, we need to show that the angle $\theta_{u, v}$ is asymptotically larger than the critical value $\bar{\theta}_{u, v}$ defined by (3.2). The following fact shows that this is the case.

Fact 6.2 Let $\beta>0$ and let $u$ and $v$ be two distinct vertices of the graph with $t_{u}, t_{v}<$ $R / 2-\omega(N)$. Then, uniformly for any such $t_{u}$ and $t_{v}$, we have

$$
\tilde{\theta}_{u, w}+\tilde{\theta}_{v, w} \gg \bar{\theta}_{u, v} .
$$

Proof. Starting from the definitions of $\tilde{\theta}_{u, w}$ and $\tilde{\theta}_{v, w}$ (see (6.3)), we have for any $\beta>0$ :

$$
\begin{aligned}
\left(\tilde{\theta}_{u, w}+\tilde{\theta}_{v, w}\right)^{2} & \geq \frac{1}{\omega(N)^{2}}\left(A_{u, w}^{-1}+A_{v, w}^{-1}\right)^{2} \geq \frac{1}{\omega(N)^{2}}\left(A_{u, w}^{-2}+A_{v, w}^{-2}\right) \\
& =\frac{v^{2}}{\omega(N)^{2}}\left(\frac{e^{\zeta\left(t_{u}+t_{w}\right)}}{N^{2}}+\frac{e^{\zeta\left(t_{v}+t_{w}\right)}}{N^{2}}\right) \geq \frac{v^{2}}{\omega(N)^{2}}\left(\frac{e^{\zeta t_{u}}}{N^{2}}+\frac{e^{\zeta t_{v}}}{N^{2}}\right) .
\end{aligned}
$$


Hence, the statement of the lemma holds if

$$
\frac{1}{\omega(N)^{2}}\left(\frac{e^{\zeta t_{u}}}{N^{2}}+\frac{e^{\zeta t_{v}}}{N^{2}}\right) \gg \frac{e^{2 \zeta t_{u}}}{N^{4}}+\frac{e^{2 \zeta t_{v}}}{N^{4}} .
$$

It suffices to show that

$$
\frac{1}{\omega(N)^{2}} \frac{e^{\zeta t_{u}}}{N^{2}} \gg \frac{e^{2 \zeta t_{u}}}{N^{4}} \quad \text { and } \quad \frac{1}{\omega(N)^{2}} \frac{e^{\zeta t_{v}}}{N^{2}} \gg \frac{e^{2 \zeta t_{v}}}{N^{4}} .
$$

The first condition (the argument for the second is identical) is equivalent to

$$
\frac{1}{\omega(N)^{2}} \gg \frac{e^{\zeta t_{u}}}{N^{2}} \Leftrightarrow \frac{1}{\omega(N)} \gg \frac{e^{\frac{\zeta}{2} t_{u}}}{N} .
$$

The latter holds as $t_{u}<R / 2-\omega(N)$.

Now, since $C=1+o(1)$ uniformly for all $\tilde{\theta}_{u, w} \leq \theta_{u, w} \leq \hat{\theta}_{u, w}$ and $\tilde{\theta}_{v, w} \leq \theta_{v, w} \leq \hat{\theta}_{v, w}$, the last integral in (6.10) is equal to:

$$
\begin{aligned}
& \int_{\tilde{\theta}_{u, w}}^{\hat{\theta}_{u, w}} \int_{-\hat{\theta}_{v, w}}^{-\tilde{\theta}_{v, w}} \frac{1}{C A_{u, w}^{\beta} \sin ^{\beta}\left(\theta_{u, w} / 2\right)+1} \frac{1}{C A_{v, w}^{\beta} \sin ^{\beta}\left(\theta_{v, w} / 2\right)+1} \\
& \times \frac{1}{C A_{u, v}^{\beta} \sin ^{\beta}\left(\left(\theta_{u, w}+\theta_{v, w}\right) / 2\right)+1} d \vartheta_{v, w} d \vartheta_{u, w} .
\end{aligned}
$$

To bound this integral we will use a first-order approximation of the $\sin (\cdot)$ function:

$$
\frac{\theta}{\pi} \leq \sin \left(\frac{\theta}{2}\right) \leq \frac{\theta}{2}
$$

Moreover, if $\theta$ is sufficiently small, then the upper bound is a tight approximation. More precisely, for any $\delta>0$, there exists $\varepsilon>0$ such that for every $\theta<\varepsilon$, we have

$$
\sin \left(\frac{\theta}{2}\right)>(1-\delta) \frac{\theta}{2} .
$$

Hence, the preceding integral can be bounded from above and below by integrals of the form

$$
\begin{aligned}
& \int_{\tilde{\theta}_{u, w}}^{\hat{\theta}_{u, w}} \int_{-\hat{\theta}_{v, w}}^{-\tilde{\theta}_{v, w}} \frac{1}{\left(\lambda A_{u, w}^{\beta}\left(\theta_{u, w} / 2\right)^{\beta}+1\right)} \frac{1}{\left(\lambda A_{v, w}^{\beta}\left(\theta_{v, w} / 2\right)^{\beta}+1\right)} \\
& \times \frac{1}{\left(\lambda A_{u, v}^{\beta}\left(\left(\theta_{u, w}+\theta_{v, w}\right) / 2\right)^{\beta}+1\right)} d \vartheta_{v, w} d \vartheta_{u, w},
\end{aligned}
$$

where $\lambda>0$ is constant when $\beta \leq 1$, but in fact $\lambda=1+o(1)$, when $\beta>1$. For $\beta \leq 1$, we will need only the upper bound, which is obtained using the lower bound in (6.11). Hence, in this case, $\lambda=1 / \pi^{\beta}$. In other words, we can take

$$
\lambda=\left\{\begin{array}{ll}
1, & \text { if } \beta>1 \\
\frac{1}{\pi^{\beta}}, & \text { if } \beta \leq 1
\end{array} .\right.
$$


At this point, we can make a convenient change of variables, setting

$$
z_{1}:=\lambda^{1 / \beta} A_{u, w} \frac{\theta_{u, w}}{2}, \quad \text { and } \quad z_{2}:=\lambda^{1 / \beta} A_{v, w} \frac{\theta_{v, w}}{2} .
$$

Note that for each triple of vertices $u, v, w$ we have

$$
\begin{aligned}
C_{w, v} & =\frac{A_{u, v}}{A_{u, w}}=e^{\frac{\zeta}{2}\left(t_{w}-t_{v}\right)}, & C_{w, u} & =\frac{A_{u, v}}{A_{v, w}}=e^{\frac{\zeta}{2}\left(t_{w}-t_{u}\right)}, \\
C_{v, w} & =\frac{A_{u, w}}{A_{u, v}}=e^{\frac{\zeta}{2}\left(t_{v}-t_{w}\right)}, & C_{v, u} & =\frac{A_{u, w}}{A_{v, w}}=e^{\frac{\zeta}{2}\left(t_{v}-t_{u}\right)}, \\
C_{u, w} & =\frac{A_{v, w}}{A_{u, v}}=e^{\frac{\zeta}{2}\left(t_{u}-t_{w}\right)}, & C_{u, v} & =\frac{A_{v, w}}{A_{u, w}}=e^{\frac{\zeta}{2}\left(t_{u}-t_{v}\right)} .
\end{aligned}
$$

Thus, we obtain: For $\beta>1$,

$$
\begin{aligned}
\mathbb{E} & \left(T(u, v ; w) \mathbf{1}_{\left\{\tilde{\theta}_{u, w} \leq \vartheta_{u, w} \leq \hat{\theta}_{u, w},-\hat{\theta}_{v, w} \leq \vartheta_{v, w} \leq-\tilde{\theta}_{v, w}\right\}} \mid t_{u}, t_{v}, t_{w}\right) \\
= & \frac{(1+o(1))}{\pi^{2} A_{u, w} A_{v, w}} \int_{\omega(N)^{-1} / 2}^{\omega(N) / 2} \int_{\omega(N)^{-1} / 2}^{\omega(N) / 2} \frac{1}{z_{1}^{\beta}+1} \frac{1}{z_{2}^{\beta}+1} \frac{d z_{1} d z_{2}}{\left(C_{w, v} z_{1}+C_{w, u} z_{2}\right)^{\beta}+1} \\
= & \frac{(1+o(1))}{\pi^{2} A_{u, w} A_{v, w}} \int_{0}^{\infty} \int_{0}^{\infty} \frac{1}{z_{1}^{\beta}+1} \frac{1}{z_{2}^{\beta}+1} \frac{1}{\left(C_{w, v} z_{1}+C_{w, u} z_{2}\right)^{\beta}+1} d z_{1} d z_{2},
\end{aligned}
$$

(where the last equality follows from the fact that the latter integral is finite).

For $\beta=1$,

$$
\begin{aligned}
& \mathbb{E}\left(T(u, v ; w) \mathbf{1}_{\left\{\tilde{\theta}_{u, w} \leq \vartheta_{u, w} \leq \hat{\theta}_{u, w},-\hat{\theta}_{v, w} \leq \vartheta_{v, w} \leq-\tilde{\theta}_{v, w}\right\}} \mid t_{u}, t_{v}, t_{w}\right) \\
& \quad \lesssim \frac{1}{A_{u, w} A_{v, w}} \int_{1 /(2 \pi \omega(N))}^{A_{v, w} / 2} \int_{1 /(2 \pi \omega(N))}^{A_{u, w} / 2} \frac{1}{z_{1}+1} \frac{1}{z_{2}+1} \frac{1}{C_{w, v} z_{1}+C_{w, u} z_{2}+1} d z_{1} d z_{2} .
\end{aligned}
$$

Finally, for $\beta<1$, we have

$$
\begin{aligned}
& \mathbb{E}\left(T(u, v ; w) \mathbf{1}_{\left\{\tilde{\theta}_{u, w} \leq \vartheta_{u, w} \leq \hat{\theta}_{u, w},-\hat{\theta}_{v, w} \leq \vartheta_{v, w} \leq-\tilde{\theta}_{v, w}\right\}} \mid t_{u}, t_{v}, t_{w}\right) \\
& \quad \lesssim \frac{1}{A_{u, w} A_{v, w}} \int_{\omega(N) / 2 \pi}^{A_{v, w} / 2} \int_{\omega(N) / 2 \pi}^{A_{u, w} / 2} \frac{1}{z_{1}^{\beta}+1} \frac{1}{z_{2}^{\beta}+1} \frac{1}{\left(C_{w, v} z_{1}+C_{w, u} z_{2}\right)^{\beta}+1} d z_{1} d z_{2} .
\end{aligned}
$$

The proof of the lemma is now complete.

We now focus on the second term in (6.4). Recall the definition of $\vartheta(u, v ; w)$ from (6.5).

Lemma 6.3. Recall the definitions of $\beta^{\prime}$ and $\delta$ from (4.1) and (4.2), respectively, and let $u, v, w \in \mathcal{D}_{R}$ be three distinct vertices. Then, uniformly for $t_{u}, t_{v}, t_{w}<R / 2-\omega(N)$,

$$
\mathbb{E}\left(T(u, v ; w)(1-\vartheta(u, v ; w)) \mid t_{u}, t_{v}, t_{w}\right)=o\left(\frac{\left(\ln A_{v, w}\right)^{\delta}}{\left(A_{u, w} A_{v, w}\right)^{\beta^{\prime}}}\right) .
$$


Proof. Using the union bound, we can bound this term as follows:

$$
\begin{aligned}
\mathbb{E}( & \left.(u, v ; w)\left(1-\mathbf{1}_{\left\{\tilde{\theta}_{u, w} \leq \vartheta_{u, w} \leq \hat{\theta}_{u, w},-\tilde{\theta}_{v, w} \geq \vartheta_{v, w} \geq-\hat{\theta}_{v, w}\right\}}\right) \mid t_{u}, t_{v}, t_{w}\right) \\
\leq & \mathbb{E}\left(T(u, v ; w) \mathbf{1}_{\left\{\vartheta_{u, w}>\hat{\theta}_{u, w}\right\}} \mid t_{u}, t_{v}, t_{w}\right) \\
& +\mathbb{E}\left(T(u, v ; w) \mathbf{1}_{\left\{\vartheta_{v, w}<-\hat{\theta}_{v, w}\right\}} \mid t_{u}, t_{v}, t_{w}\right) \\
& +\mathbb{E}\left(T(u, v ; w) \mathbf{1}_{\left\{0<\vartheta_{u, w}<\tilde{\theta}_{u, w}\right\}} \mid t_{u}, t_{v}, t_{w}\right) \\
& +\mathbb{E}\left(T(u, v ; w) \mathbf{1}_{\left\{0>\vartheta_{v, w}>-\tilde{\theta}_{v, w}\right\}} \mid t_{u}, t_{v}, t_{w}\right) .
\end{aligned}
$$

We now obtain upper bounds on each term in the right-hand side of (6.13).

We bound the first term as follows:

$$
\mathbb{P}\left(u \sim w, \hat{\theta}_{u, w}<\vartheta_{u, w} \leq \pi \mid t_{u}, t_{v}, t_{w}\right)=\frac{1}{2 \pi} \int_{\hat{\theta}_{u, w}}^{\pi} p_{u, w} d \vartheta_{u, w} .
$$

When $\beta \leq 1$, by (6.3) we have

$$
\int_{\hat{\theta}_{u, w}}^{\pi} p_{u, w} d \vartheta_{u, w} \leq \tilde{\theta}_{u, w}=\left\{\begin{array}{ll}
\frac{1}{\omega(N) A_{u, v}}=o\left(\frac{1}{A_{u, w}}\right), & \text { if } \beta=1 \\
\frac{\omega(N)}{A_{u, v}}=o\left(\frac{1}{A_{u, w}^{\beta}}\right), & \text { if } \beta<1
\end{array} .\right.
$$

For $\beta>1$, we need to work slightly more. We have

$$
\begin{aligned}
& \mathbb{P}\left(u \sim w, \hat{\theta}_{u, w}<\vartheta_{u, w} \leq \pi \mid t_{u}, t_{v}, t_{w}\right)=\frac{1}{2 \pi} \int_{\hat{\theta}_{u, w}}^{\pi} p_{u, w} d \vartheta_{u, w} \\
& \stackrel{16.6)}{=} \frac{1}{2 \pi} \int_{\hat{\theta}_{u, w}}^{\pi} \frac{1}{C A_{u, w}^{\beta} \sin ^{\beta}\left(\theta_{u, w} / 2\right)+1} d \vartheta_{u, w} \leq \frac{1+o(1)}{2 \pi A_{u, w}^{\beta}} \int_{\hat{\theta}_{u, w}}^{\pi} \frac{d \vartheta_{u, w}}{\sin ^{\beta}\left(\theta_{u, w} / 2\right),}
\end{aligned}
$$

because $C=1+o(1)$ uniformly for $u, v$. Using the well-known inequality

$$
\sin \left(\frac{\theta}{2}\right) \geq \frac{\theta}{\pi} \quad \text { for all } \theta \in[0, \pi],
$$

we can bound the previous term by

$$
\begin{aligned}
& \frac{1}{2 \pi C A_{u, w}^{\beta}} \int_{\hat{\theta}_{u, w}}^{\pi} \frac{1}{\sin ^{\beta}\left(\theta_{u, w} / 2\right)} d \theta_{u, w} \leq \frac{\pi^{\beta-1}}{2 C A_{u, w}^{\beta}} \int_{\hat{\theta}_{u, w}}^{\pi} \theta_{u, w}^{-\beta} d \theta_{u, w} \\
& =\frac{\pi^{\beta-1}}{2 C(\beta-1) A_{u, w}^{\beta}}\left(\hat{\theta}_{u, w}^{-\beta+1}-\pi^{-\beta+1}\right) \asymp \frac{1}{A_{u, w}^{\beta}} \frac{A_{u, w}^{\beta-1}}{\omega(N)^{\beta-1}} \asymp \frac{A_{u, w}^{-1}}{\omega(N)^{\beta-1}} .
\end{aligned}
$$

Hence, for $\beta>1$, we have that

$$
\mathbb{P}\left(u \sim w, \vartheta_{u, w}>\hat{\theta}_{u, w} \mid t_{u}, t_{v}, t_{w}\right) \lesssim \frac{1}{A_{u, w} \omega(N)^{\beta-1}} .
$$

We can now return to (6.13) and use the above estimates to bound the first term of the 
right-hand side:

$$
\begin{aligned}
& \mathbb{E}\left(T(u, v ; w) \mathbf{1}_{\vartheta_{u, w}>\hat{\theta}_{u, w}} \mid t_{u}, t_{v}, t_{w}\right) \leq \mathbb{P}\left(u \sim w, v \sim w, \vartheta_{u, w}>\hat{\theta}_{u, w} \mid t_{u}, t_{v}, t_{w}\right) \\
& \quad=\mathbb{P}\left(u \sim w, \vartheta_{u, w}>\hat{\theta}_{u, w} \mid t_{u}, t_{v}, t_{w}\right) \mathbb{P}\left(v \sim w \mid t_{u}, t_{v}, t_{w}\right) \\
& \qquad \begin{array}{ll}
o\left(\frac{1}{A_{u, w} A_{v, w}}\right), & \text { if } \beta>1 \\
o\left(\frac{\ln \left(A_{v, w}\right)}{A_{u, w} A_{v, w}}\right), & \text { if } \beta=1 . \\
o\left(\frac{1}{A_{u, w}^{\beta} A_{v, w}^{\beta}}\right), & \text { if } \beta<1
\end{array}
\end{aligned}
$$

The first equality is due to the independence of the relative positions of $u$ and $v$ with respect to $w$, together with the independence of the edges, given the positions of the vertices. The very same calculation can be used in order to deduce the same bound on $\mathbb{E}\left(T(u, v ; w) \mathbf{1}_{\left\{\vartheta_{v, w}<-\hat{\theta}_{v, w}\right\}} \mid t_{u}, t_{v}, t_{w}\right)$.

Now, we use the fact that $p_{u, w}(\theta) \leq 1$ to bound

$$
\begin{aligned}
\mathbb{E}\left(T(u, v ; w) \mathbf{1}_{\left\{\vartheta_{u, w}<\tilde{\theta}_{u, w}\right\}} \mid t_{u}, t_{v}, t_{w}\right) & \leq \mathbb{P}\left(v \sim w, 0<\vartheta_{u, w}<\tilde{\theta}_{u, w} \mid t_{u}, t_{v}, t_{w}\right) \\
& =\mathbb{P}\left(v \sim w \mid t_{u}, t_{v}, t_{w}\right) \mathbb{P}\left(0<\vartheta_{u, w}<\tilde{\theta}_{u, w} \mid t_{u}, t_{v}, t_{w}\right) \\
& =\left\{\begin{array}{ll}
o\left(A_{u, w}^{-1} A_{v, w}^{-1}\right), & \text { if } \beta \geq 1 \\
o\left(A_{u, w}^{-\beta} A_{v, w}^{-\beta}\right), & \text { if } \beta<1
\end{array} .\right.
\end{aligned}
$$

In the last two equalities, we used the fact that the event $\{v \sim w\}$ is independent of the event $\left\{\vartheta_{u, w}<\tilde{\theta}_{u, w}\right\}$ together with Lemma 3.1. The same bound can be deduced for $\mathbb{E}\left(T(u, v ; w) \mathbf{1}_{\left\{0>\vartheta_{v, w}>-\tilde{\theta}_{v, w}\right\}} \mid t_{u}, t_{v}, t_{w}\right)$.

Lemma 6.3 implies the following.

Corollary 6.4. For $\beta>0$, we have

$$
\mathbb{E}\left(T(u, v ; w)(1-\vartheta(u, v ; w)) \mid t_{u}, t_{v}, t_{w}\right)=o\left(\mathbb{P}\left(u \sim w, v \sim w \mid t_{u}, t_{v}, t_{w}\right)\right),
$$

uniformly over all $t_{u}, t_{v}, t_{w} \leq R / 2-\omega(N)$.

Hence, for any $\beta>0$, the contribution of these terms to $\mathbb{E}\left(\widehat{T}^{\prime}\right)$ is $o(\mathbb{E}(\widehat{\Lambda}))$.

Thus, we need to focus on the terms that are covered by Lemma 6.1.

Using (6.12), we write

$$
\frac{1}{A_{u, w} A_{v, w}}=\left(\frac{v}{N}\right)^{2} \frac{e^{2 \zeta t_{w}}}{C_{w, v} C_{w, u}} .
$$

Thus, we can rewrite the right-hand sides of (6.7), (6.8), and (6.9) as follows:

$$
I(u, v ; w):=\left(\frac{v}{N}\right)^{2} \frac{e^{2 \zeta t_{w}}}{C_{w, v} C_{w, u}} \int_{D_{z}} \frac{1}{z_{1}^{\beta}+1} \frac{1}{z_{2}^{\beta}+1} \frac{d z_{1} d z_{2}}{\left(C_{w, v} z_{1}+C_{w, u} z_{2}\right)^{\beta}+1},
$$


where $D_{z}$ is the domain where $z_{1}$ and $z_{2}$ range; we have

$$
D_{z}= \begin{cases}{[0, \infty)^{2},} & \text { if } \beta>1 \\ {\left[(2 \pi \omega(N))^{-1}, A_{v, w} / 2\right] \times\left[(2 \pi \omega(N))^{-1}, A_{u, w} / 2\right],} & \text { if } \beta=1 \\ {\left[\left(\omega(N) /(2 \pi), A_{v, w} / 2\right] \times\left[\omega(N) /(2 \pi), A_{u, w} / 2\right],\right.} & \text { if } \beta<1\end{cases}
$$

Together with Corollary 6.4, this implies the following statement.

Lemma 6.5. For any distinct $u, v, w \in \mathrm{V}_{N}$, we have the following: if $\beta>1$,

$$
\mathbb{E}(T(u, v ; w) \vartheta(u, v ; w))=(1+o(1)) \int_{D_{t}} I(u, v ; w) \bar{\rho}_{N}\left(t_{u}\right) \bar{\rho}_{N}\left(t_{v}\right) \bar{\rho}_{N}\left(t_{w}\right) d t_{u} d t_{v} d t_{w} ;
$$

whereas if $\beta \leq 1$,

$$
\mathbb{E}(T(u, v ; w) \vartheta(u, v ; w)) \lesssim \int_{D_{t}} I(u, v ; w) \bar{\rho}_{N}\left(t_{u}\right) \bar{\rho}_{N}\left(t_{v}\right) \bar{\rho}_{N}\left(t_{w}\right) d t_{u} d t_{v} d t_{w},
$$

where $D_{t}:=[0, R / 2-\omega(N)]^{3}$. Also, for any $\beta>0$,

$$
N^{3} \mathbb{E}(T(u, v ; w)(1-\vartheta(u, v ; w)))=o(\mathbb{E}(\widehat{\Lambda})) .
$$

\subsection{Proof of Theorem 1.3}

Recall that $\beta>1$. Let $t<R$ be a positive constant, and consider $N$ and $R$ so large, that $R / 2-\omega(N)>t$. Let $\widehat{T}_{t}$ denote the number of triangles in $\mathcal{G}(N ; \zeta, \alpha, \beta, v)$ whose vertices have type at most $t$. Note that, in this part, the triangles are automatically typical, hence $\widehat{T}_{t}=T_{t}$ a.a.s. Similarly, we let $\widehat{\Lambda}_{t}$ denote the number of incomplete triangles all of whose vertices have type at most $t$. For three distinct vertices $u, v$, and $w$, we let $T_{t}(u, v ; w)$ be defined as $T(u, v ; w)$ but with the additional restriction that $t_{u}, t_{v}, t_{w} \leq t$.

Finally, let $\widehat{T}_{t}^{\prime}$ be defined as $\widehat{T}^{\prime}$ with the variables $T(u, v ; w)$ replaced by $T_{t}(u, v ; w)$. Note that the analogue of (6.2) holds between $\widehat{T}_{t}$ and $\widehat{T}_{t}^{\prime}$.

By Lemma 3.1, it follows that

$$
\begin{gathered}
\mathbb{E}\left(\widehat{\Lambda}_{t}\right)=(1+o(1)) 3\left(\begin{array}{c}
N \\
3
\end{array}\right)\left(C_{\beta}\right)^{2} \int_{[0, t]^{3}} \frac{1}{A_{w, u} A_{w, v}} \bar{\rho}_{N}\left(t_{u}\right) \bar{\rho}_{N}\left(t_{v}\right) \bar{\rho}_{N}\left(t_{w}\right) d t_{u} d t_{v} d t_{w} \\
\stackrel{\text { Claim }}{=}{ }^{3.4}(1+o(1)) \frac{1}{2} N\left(v C_{\beta}\right)^{2} \alpha^{3} \int_{[0, t]^{3}} e^{\frac{\zeta}{2}\left(t_{u}+t_{v}\right)+\zeta t_{w}} e^{-\alpha\left(t_{u}+t_{v}+t_{w}\right)} d t_{u} d t_{v} d t_{w}
\end{gathered}
$$

One can show that $\widehat{\Lambda}_{t}$ is concentrated around its expected value through a second moment argument that is very similar to that in Section 5, which we omit. Thus,

$$
\widehat{\Lambda}_{t}=\mathbb{E}\left(\widehat{\Lambda}_{t}\right)\left(1+o_{p}(1)\right) .
$$

With regard to $\widehat{T}_{t}$, we use the following fact. 
Claim 6.6. For $\beta>1$ and for $N$ large enough, we have

$$
\widehat{T}_{t}^{\prime}-\widehat{T}_{t} \leq \sum_{w \in \mathrm{V}_{N}} \sum_{\substack{(u, v): \\ u, v \in \mathrm{V}_{N} \backslash\{w\}}} T_{t}(u, v ; w)(1-\vartheta(u, v ; w)) .
$$

Proof. We write

$$
\begin{aligned}
\widehat{T}_{t}^{\prime} & =\sum_{w \in \mathrm{V}_{N}} \sum_{\substack{(u, v): \\
u, v \in \mathrm{V}_{N} \backslash\{w\}}} T_{t}(u, v ; w) \\
& =\sum_{w \in \mathrm{V}_{N}} \sum_{\substack{(u, v): \\
u, v \in \mathrm{V}_{N} \backslash\{w\}}} T_{t}(u, v ; w)(\vartheta(u, v ; w)+1-\vartheta(u, v ; w)) .
\end{aligned}
$$

The definitions in (6.3) imply that, for $\beta>1$, we have $\hat{\theta}_{u, w}, \hat{\theta}_{v, w}, \hat{\theta}_{u, v}=o(1)$. Therefore, for $N$ sufficiently large, if $\vartheta(u, v ; w)=1$, then $\vartheta(u, w ; v)=\vartheta(v, w ; u)=0$. Furthermore, if $\vartheta(u, v ; w)=1$, then $\vartheta(v, u ; w)=0$ and also $\vartheta(w, u ; v)=\vartheta(w, v ; u)=0$.

Hence, if $T_{t}(u, v, w)$ denotes the indicator random variable that is equal to 1 if and only if the vertices $u, v, w$ form a triangle and all have types at most $t$, we have

$$
\begin{aligned}
\widehat{T}_{t} & \geq \sum_{w \in \mathrm{V}_{N}} \sum_{\substack{u, v): \\
u, v \in \mathrm{V}_{N} \backslash\{w\}}} T_{t}(u, v, w) \vartheta(u, v ; w) \\
& \geq \sum_{w \in \mathrm{V}_{N}} \sum_{\substack{u, v) ; \\
u, v \in \mathrm{V}_{N} \backslash\{w\}}} T_{t}(u, v ; w) \vartheta(u, v ; w) .
\end{aligned}
$$

Now, subtracting (6.20) from (6.19), the claim follows.

Therefore, by Corollary 6.4, we have

$$
\mathbb{E}\left(\widehat{T}_{t}^{\prime}-\widehat{T}_{t}\right)=o\left(\mathbb{E}\left(\widehat{\Lambda}_{t}\right)\right) .
$$

Now, by (6.7) in Lemma 6.1 we have

$$
\begin{aligned}
\mathbb{E}\left(\widehat{T}_{t}^{\prime}\right)= & (1+o(1)) 6\left(\begin{array}{c}
N \\
3
\end{array}\right) \frac{1}{\pi^{2}} \int_{[0, t]^{3}} I(u, v ; w) \bar{\rho}_{N}\left(t_{u}\right) \bar{\rho}_{N}\left(t_{v}\right) \bar{\rho}_{N}\left(t_{w}\right) d t_{u} d t_{v} d t_{w} \\
\stackrel{\text { Claim }}{=}{ }^{3.4} & (1+o(1)) N\left(\frac{v}{\pi}\right)^{2} \alpha^{3} \int_{[0, t]^{3}} e^{\frac{\zeta}{2}\left(t_{u}+t_{v}\right)+\zeta t_{w}} e^{-\alpha\left(t_{u}+t_{v}+t_{w}\right)} \\
& \times\left[\int_{D_{z}} \frac{1}{z_{1}^{\beta}+1} \frac{1}{z_{2}^{\beta}+1} \frac{1}{\left(C_{w, v} z_{1}+C_{w, u} z_{2}\right)^{\beta}+1} d z_{1} d z_{2}\right] d t_{u} d t_{v} d t_{w} .
\end{aligned}
$$

The concentration of $\widehat{T}_{t}$ around its expected value can be shown using a second moment argument similar to that used in Lemma 5.3 (we omit the details), from which we deduce that

$$
\widehat{T}_{t}=\mathbb{E}\left(\widehat{T}_{t}\right)\left(1+o_{p}(1)\right) .
$$


But (6.21) implies that $\mathbb{E}\left(\widehat{T}_{t}\right)=\mathbb{E}\left(\widehat{T}_{t}^{\prime}\right)+o\left(\mathbb{E}\left(\widehat{\Lambda}_{t}\right)\right)$. Because $\mathbb{E}\left(\widehat{\Lambda}_{t}\right)=\Theta\left(\mathbb{E}\left(\widehat{T}_{t}^{\prime}\right)\right)$, we deduce that

$$
\widehat{T}_{t}=\mathbb{E}\left(\widehat{T}_{t}^{\prime}\right)\left(1+o_{p}(1)\right) .
$$

This, combined with (6.18), implies the statement of Theorem 1.3.

The value of $L_{\infty}(\beta, \zeta, \alpha)$ is also deduced as above using Proposition 2.3 and taking $t=R / 2-\omega(N)$, which is equivalent (up to a $1+o(1)$ factor) to taking the integrals up to $t=\infty$.

\section{PROOF OF PROPOSITION 2.2}

In this section, we prove separately the results for $\beta>1, \beta=1$, and $\beta<1$. For $\beta \leq 1$, we will give only an upper bound for $\mathbb{E}(\widehat{T})$ (cf. Sections 7.2, 7.3). For $\beta>1$, we will consider two cases, namely $\zeta / \alpha<1$ and $\zeta / \alpha \geq 1$. In the former, we will show that $\mathbb{E}(\widehat{T}) \asymp \mathbb{E}(\widehat{\Lambda})$. Note that the upper bound holds trivially, as $3 \widehat{T} \leq \widehat{\Lambda}$. We will deduce only a matching lower bound in the next section. For $\zeta / \alpha \geq 1$, we will deduce an upper bound.

\subsection{Proof of Proposition 2.2(i) $(\beta>1)$}

Case $1 \leq \zeta / \alpha<1$. We will deduce a lower bound on $\mathbb{E}(T(u, v ; w) \vartheta(u, v ; w))$ integrating $I(u, v ; w)$ over the sub domain of $D_{t}$, which is $D_{t}^{\prime}:=\left\{\left(t_{u}, t_{v}, t_{w}\right): 0<t_{u}, t_{v}<t_{w}\right\}$. Note that, in this case, $C_{w, u}, C_{w, v}>1$. Hence, we can bound from below the double integral that appears in (6.16) as follows:

$$
\begin{aligned}
& \int_{0}^{\infty} \int_{0}^{\infty} \frac{1}{z_{1}^{\beta}+1} \frac{1}{z_{2}^{\beta}+1} \frac{1}{\left(C_{w, v} z_{1}+C_{w, u} z_{2}\right)^{\beta}+1} d z_{1} d z_{2} \\
& \geq \int_{0}^{\infty} \int_{0}^{\infty} \frac{1}{\left(C_{w, v} z_{1}\right)^{\beta}+1} \frac{1}{\left(C_{w, u} z_{2}\right)^{\beta}+1} \frac{1}{\left(C_{w, v} z_{1}+C_{w, u} z_{2}\right)^{\beta}+1} d z_{1} d z_{2} \\
& =\frac{1}{C_{w, v} C_{w, u}} \int_{0}^{\infty} \int_{0}^{\infty} \frac{1}{x_{1}^{\beta}+1} \frac{1}{x_{2}^{\beta}+1} \frac{1}{\left(x_{1}+x_{2}\right)^{\beta}+1} d x_{1} d x_{2} .
\end{aligned}
$$

Therefore,

$$
I(u, v ; w) \geq\left(\frac{v}{N}\right)^{2} \frac{e^{2 \zeta t_{w}}}{C_{w, v}^{2} C_{w, u}^{2}} \int_{0}^{\infty} \int_{0}^{\infty} \frac{1}{x_{1}^{\beta}+1} \frac{1}{x_{2}^{\beta}+1} \frac{1}{\left(x_{1}+x_{2}\right)^{\beta}+1} d x_{1} d x_{2},
$$

which in turn yields

$$
\begin{aligned}
\mathbb{E}(T(u, v ; w) \vartheta(u, v ; w)) \geq & \left(\frac{v}{N}\right)^{2}\left[\int_{[0, \infty)^{2}} \frac{1}{x_{1}^{\beta}+1} \frac{1}{x_{2}^{\beta}+1} \frac{d x_{1} d x_{2}}{\left(x_{1}+x_{2}\right)^{\beta}+1}\right] \\
& \times \int_{D_{t}^{\prime}} \frac{e^{2 \zeta t_{w}}}{C_{w, v}^{2} C_{w, u}^{2}} \bar{\rho}_{N}\left(t_{u}\right) \bar{\rho}_{N}\left(t_{v}\right) \bar{\rho}_{N}\left(t_{w}\right) d t_{u} d t_{v} d t_{w} .
\end{aligned}
$$


We will show that for $\zeta / \alpha<1$, the latter integral is $\Omega(1)$. Indeed, we have

$$
\frac{e^{2 \zeta t_{w}}}{C_{w, v}^{2} C_{w, u}^{2}}=e^{\zeta\left(t_{u}+t_{v}\right)}
$$

whereby, using Claim 3.4 (for large $N$ ), we obtain

$$
\begin{gathered}
\int_{D_{t}^{\prime}} \frac{e^{2 \zeta t_{w}}}{C_{w, v}^{2} C_{w, u}^{2}} \bar{\rho}_{N}\left(t_{u}\right) \bar{\rho}_{N}\left(t_{v}\right) \bar{\rho}_{N}\left(t_{w}\right) d t_{u} d t_{v} d t_{w} \gtrsim \frac{1}{2} \int_{D_{t}^{\prime}} e^{\zeta\left(t_{u}+t_{v}\right)-\alpha\left(t_{u}+t_{v}+t_{w}\right)} d t_{u} d t_{v} d t_{w} \\
\asymp \int_{0}^{R / 2-\omega(N)} \int_{0}^{t_{w}} \int_{0}^{t_{w}} e^{(\zeta-\alpha)\left(t_{u}+t_{v}\right)-\alpha t_{w}} d t_{u} d t_{v} d t_{w} \\
=\int_{0}^{R / 2-\omega(N)}\left[\int_{0}^{t_{w}} e^{(\zeta-\alpha) t_{u}} d t_{u}\right]^{2} e^{-\alpha t_{w}} d t_{w} \gtrsim 1 .
\end{gathered}
$$

Thus, after recalling the definition of $\vartheta(u, v ; w)$ from $(6.5)$, we deduce that

$$
\mathbb{E}(T(u, v ; w) \vartheta(u, v ; w)) \gtrsim \frac{1}{N^{2}} \Rightarrow \mathbb{E}\left(\widehat{T}^{\prime}\right) \gtrsim\left(\begin{array}{l}
N \\
3
\end{array}\right) \mathbb{E}(T(u, v ; w) \vartheta(u, v ; w)) \gtrsim N .
$$

Now, (6.2) implies that

$$
\mathbb{E}(\widehat{T}) \gtrsim N
$$

Case $1 \leq \zeta / \alpha<2$. In this range, we provide an upper bound on $\mathbb{E}\left(\widehat{T}^{\prime}\right)$ and show that it is $o(\mathbb{E}(\widehat{\Lambda}))$. By (6.2), this is clearly enough to deduce the second part of Proposition 2.2(i). We write

$$
\begin{aligned}
\mathbb{E}\left(\widehat{T}^{\prime}\right) & \leq N^{3} \mathbb{E}(T(u, v ; w)) \\
& =N^{3}(\mathbb{E}(T(u, v ; w) \vartheta(u, v ; w))+\mathbb{E}(T(u, v ; w)(1-\vartheta(u, v ; w)))),
\end{aligned}
$$

where $u, v, w$ are three distinct vertices. By the second part of Lemma 6.5, the second term is $o(\mathbb{E}(\widehat{\Lambda}))$. We will also show that

$$
N^{3}(\mathbb{E}(T(u, v ; w) \vartheta(u, v ; w))=o(\mathbb{E}(\widehat{\Lambda})) .
$$

To this end, we split the domain of the integral of $I(u, v ; w)$ into three sub-domains and bound $I(u, v ; w)$ separately on each of them. In particular, we define

$$
\begin{aligned}
& D_{t}^{(1)}:=\left\{\left(t_{u}, t_{v}, t_{w}\right): t_{u}, t_{v}>t_{w}\right\} \\
& D_{t}^{(2)}:=\left\{\left(t_{u}, t_{v}, t_{w}\right): t_{u} \leq t_{w}\right\} \\
& D_{t}^{(3)}:=\left\{\left(t_{u}, t_{v}, t_{w}\right): t_{v} \leq t_{w}\right\} .
\end{aligned}
$$


It is clear that the last two sub domains are not disjoint, but because we are interested only in upper bounds, this does not create any issues. It is immediately seen that

$$
\begin{aligned}
\mathbb{E}(T(u, v ; w) \vartheta(u, v ; w)) \leq & \int_{D_{t}^{(1)}} I(u, v ; w) \bar{\rho}_{N}\left(t_{u}\right) \bar{\rho}_{N}\left(t_{v}\right) \bar{\rho}_{N}\left(t_{w}\right) d t_{u} d t_{v} d t_{w} \\
& +\int_{D_{t}^{(2)}} I(u, v ; w) \bar{\rho}_{N}\left(t_{u}\right) \bar{\rho}_{N}\left(t_{v}\right) \bar{\rho}_{N}\left(t_{w}\right) d t_{u} d t_{v} d t_{w} \\
& +\int_{D_{t}^{(3)}} I(u, v ; w) \bar{\rho}_{N}\left(t_{u}\right) \bar{\rho}_{N}\left(t_{v}\right) \bar{\rho}_{N}\left(t_{w}\right) d t_{u} d t_{v} d t_{w}
\end{aligned}
$$

We will bound from above each of these three integrals. In fact, we will do so only for the first two - the last one can be treated exactly as the second one.

To bound the first integral, we use the following upper bound on $I(u, v ; w)$ :

$$
I(u, v ; w) \leq\left(\frac{v}{N}\right)^{2} \frac{e^{2 \zeta t_{w}}}{C_{w, v} C_{w, u}} \int_{D_{z}} \frac{1}{z_{1}^{\beta}+1} \frac{1}{z_{2}^{\beta}+1} d z_{1} d z_{2} .
$$

Also,

$$
\frac{e^{2 \zeta t_{w}}}{C_{w, v} C_{w, u}}=e^{\zeta t_{w}+\frac{\zeta}{2}\left(t_{u}+t_{v}\right)}
$$

We now integrate this quantity over $D_{t}^{(1)}$ by applying Claim 3.4 as follows:

$$
\begin{aligned}
& \int_{D_{t}^{(1)}} e^{\zeta t_{w}+\frac{\zeta}{2}\left(t_{u}+t_{v}\right)} \bar{\rho}_{N}\left(t_{u}\right) \bar{\rho}_{N}\left(t_{v}\right) \bar{\rho}_{N}\left(t_{w}\right) d t_{u} d t_{v} d t_{w} \\
& \quad \lesssim \int_{0}^{R / 2-\omega(N)} \int_{t_{w}}^{R / 2-\omega(N)} \int_{t_{w}}^{R / 2-\omega(N)} e^{\zeta t_{w}+\frac{\zeta}{2}\left(t_{u}+t_{v}\right)-\alpha\left(t_{u}+t_{v}+t_{w}\right)} d t_{u} d t_{v} d t_{w} \\
& =\int_{0}^{R / 2-\omega(N)} e^{(\zeta-\alpha) t_{w}}\left[\int_{t_{w}}^{R / 2-\omega(N)} e^{(\zeta / 2-\alpha) t_{u}} d t_{u}\right]^{2} d t_{w} \\
& \quad \lesssim \int_{0}^{R / \alpha<2} e^{(\zeta-\alpha) t_{w}+2(\zeta / 2-\alpha) t_{w}} d t_{w}=\int_{0}^{R / 2-\omega(N)} e^{(2 \zeta-3 \alpha) t_{w}} d t_{w} .
\end{aligned}
$$

If $\zeta / \alpha<3 / 2$, then the above integral is $O(1)$, whereas if $\zeta / \alpha=3 / 2$, then this is $O(R)$. Finally, when $\zeta / \alpha>3 / 2$, this is $O\left(N^{2-3 \alpha / \zeta}\right)$. Hence,

$$
\int_{D_{t}^{(1)}} e^{\zeta t_{w}+\frac{\zeta}{2}\left(t_{u}+t_{v}\right)} \bar{\rho}_{N}\left(t_{u}\right) \bar{\rho}_{N}\left(t_{v}\right) \bar{\rho}_{N}\left(t_{w}\right) d t_{u} d t_{v} d t_{w} \lesssim\left\{\begin{array}{ll}
1, & \text { if } \frac{\zeta}{\alpha}=1 \\
N^{2-3 \alpha / \zeta}, & \text { if } 1<\frac{\zeta}{\alpha}<2
\end{array} .\right.
$$

Therefore,

$$
\begin{aligned}
N^{3} \int_{D_{t}^{(1)}} I(u, v ; w) \bar{\rho}_{N}\left(t_{u}\right) \bar{\rho}_{N}\left(t_{v}\right) \bar{\rho}_{N}\left(t_{w}\right) d t_{u} d t_{v} d t_{w} \\
\quad \lesssim\left\{\begin{array}{ll}
N, & \text { if } \zeta / \alpha=1 \\
N^{3-3 \alpha / \zeta}, & \text { if } 1<\zeta / \alpha<2
\end{array} .\right.
\end{aligned}
$$


Note that both quantities are $o(\mathbb{E}(\widehat{\Lambda})$ ) (to see the latter, note that $3-3 \alpha / \zeta<2-\alpha / \zeta$, which is equivalent to $1<2 \alpha / \zeta$, that is, $\zeta / \alpha<2$ ).

Now we consider the second integral in (7.2). Note that on the sub domain $D_{t}^{(2)}$, we have $C_{w, u} \geq 1$. In this case, we bound the integral in (6.16) as follows:

$$
\begin{aligned}
& \int_{0}^{\infty} \int_{0}^{\infty} \frac{1}{z_{1}^{\beta}+1} \frac{1}{z_{2}^{\beta}+1} \frac{1}{\left(C_{w, v} z_{1}+C_{w, u} z_{2}\right)^{\beta}+1} d z_{1} d z_{2} \\
& \quad \leq \int_{0}^{\infty} \int_{0}^{\infty} \frac{1}{z_{1}^{\beta}+1} \frac{1}{\left(C_{w, u} z_{2}\right)^{\beta}+1} d z_{1} d z_{2} \\
& \quad=\frac{1}{C_{w, u}} \int_{0}^{\infty} \int_{0}^{\infty} \frac{1}{z_{1}^{\beta}+1} \frac{1}{x_{2}^{\beta}+1} d z_{1} d x_{2}=\frac{1}{C_{w, u}}\left[\int_{0}^{\infty} \frac{1}{z_{1}^{\beta}+1} d z_{1}\right]^{2} .
\end{aligned}
$$

Hence,

$$
I(u, v ; w) \leq\left(\frac{v}{N}\right)^{2} \frac{e^{2 \zeta t_{w}}}{C_{w, v} C_{w, u}^{2}}\left[\int_{0}^{\infty} \frac{1}{z_{1}^{\beta}+1} d z_{1}\right]^{2}
$$

and

$$
\frac{e^{2 \zeta t_{w}}}{C_{w, v} C_{w, u}^{2}}=e^{\frac{\zeta}{2}\left(t_{w}+t_{v}\right)+\zeta t_{u}} .
$$

To bound the second integral in (7.2), we need to bound the integral of this quantity over $D_{t}^{(2)}$. Using Claim 3.4, we have

$$
\begin{aligned}
& \int_{D_{t}^{(2)}} e^{\frac{\zeta}{2}\left(t_{w}+t_{v}\right)+\zeta t_{u}} \bar{\rho}_{N}\left(t_{u}\right) \bar{\rho}_{N}\left(t_{v}\right) \bar{\rho}_{N}\left(t_{w}\right) d t_{u} d t_{v} d t_{w} \\
& \asymp \int_{0}^{R / 2-\omega(N)} \int_{0}^{R / 2-\omega(N)} e^{(\zeta / 2-\alpha)\left(t_{w}+t_{v}\right)}\left[\int_{0}^{t_{w}} e^{(\zeta-\alpha) t_{u}} d t_{u}\right] d t_{v} d t_{w} \\
& =\left[\int_{0}^{R / 2-\omega(N)} e^{(\zeta / 2-\alpha) t_{v}} d t_{v}\right] \int_{0}^{R / 2-\omega(N)} e^{(\zeta / 2-\alpha) t_{w}}\left[\int_{0}^{t_{w}} e^{(\zeta-\alpha) t_{u}} d t_{u}\right] d t_{w} \\
& \qquad \check{\asymp<2} \int_{0}^{R / 2-\omega(N)} e^{(\zeta / 2-\alpha) t_{w}}\left[\int_{0}^{t_{w}} e^{(\zeta-\alpha) t_{u}} d t_{u}\right] d t_{w} .
\end{aligned}
$$

Now, when $\zeta / \alpha=1$, this yields:

$$
\int_{D_{t}^{(2)}} e^{\frac{\zeta}{2}\left(t_{w}+t_{v}\right)+\zeta t_{u}} \bar{\rho}_{N}\left(t_{u}\right) \bar{\rho}_{N}\left(t_{v}\right) \bar{\rho}_{N}\left(t_{w}\right) d t_{u} d t_{v} d t_{w} \asymp \int_{0}^{R / 2-\omega(N)} t_{w} e^{(\zeta / 2-\alpha) t_{w}} d t_{w} \asymp 1,
$$

which, by (7.4), implies that

$$
N^{3} \int_{D_{t}^{(2)}} I(u, v ; w) \bar{\rho}_{N}\left(t_{u}\right) \bar{\rho}_{N}\left(t_{v}\right) \bar{\rho}_{N}\left(t_{w}\right) d t_{u} d t_{v} d t_{w} \lesssim N=o(\mathbb{E}(\widehat{\Lambda}))
$$


If $1<\zeta / \alpha<2$, then

$$
\begin{gathered}
\int_{D_{t}^{(2)}} e^{\frac{\zeta}{2}\left(t_{w}+t_{v}\right)+\zeta t_{u}} \bar{\rho}_{N}\left(t_{u}\right) \bar{\rho}_{N}\left(t_{v}\right) \bar{\rho}_{N}\left(t_{w}\right) d t_{u} d t_{v} d t_{w} \\
\quad \lesssim \int_{0}^{R / 2-\omega(N)} e^{(3 \zeta / 2-2 \alpha) t_{w}} d t_{w} \asymp \begin{cases}1, & \text { if } 1<\zeta / \alpha<4 / 3 \\
R, & \text { if } \zeta / \alpha=4 / 3 \\
N^{3 / 2-2 \alpha / \zeta}, & \text { if } 4 / 3<\zeta / \alpha<2\end{cases}
\end{gathered}
$$

Therefore,

$$
N^{3} \int_{D_{t}^{(2)}} I(u, v ; w) \bar{\rho}_{N}\left(t_{u}\right) \bar{\rho}_{N}\left(t_{v}\right) \bar{\rho}_{N}\left(t_{w}\right) d t_{u} d t_{v} d t_{w} \lesssim R N^{5 / 2-2 \alpha / \zeta} .
$$

The latter is $o(\mathbb{E}(\widehat{\Lambda})$ ), since $5 / 2-2 \alpha / \zeta<2-\alpha / \zeta$ (which is equivalent to $1 / 2<\alpha / \zeta$, that is, $\zeta / \alpha<2$ ). Hence, (7.3), (7.5), (7.6), together with (7.2), imply (7.1).

Conjecture 7.1. From the calculations seen in this section, we get two possible values for $\zeta / \alpha$ for which the probability of triangles might have a sharp phase transition. We conjecture that the value in which this is happening is $\zeta / \alpha=3 / 2$.

\subsection{Proof of Proposition 2.2(ii) $(\beta=1)$}

In this section, we will prove (2.5) for the case $\beta=1$. The second part of Lemma 6.5 implies

$$
\mathbb{E}\left(\widehat{T}^{\prime}\right)=o(\mathbb{E}(\widehat{\Lambda}))
$$

which, by (6.2), implies Proposition 2.2(ii). We first bound the integral on the right-hand side of (6.16) as follows:

$$
\begin{aligned}
\int_{D_{z}} & \frac{1}{z_{1}+1} \frac{1}{z_{2}+1} \frac{1}{C_{w, v} z_{1}+C_{w, u} z_{2}+1} d z_{1} d z_{2} \\
\quad= & \int_{1 /(2 \pi \omega(N))}^{A_{v, w} / 2} \int_{1 /(2 \pi \omega(N))}^{A_{u, w} / 2} \frac{1}{z_{1}+1} \frac{1}{z_{2}+1} \frac{1}{C_{w, v} z_{1}+C_{w, u} z_{2}+1} d z_{1} d z_{2} \\
\leq & \int_{1 /(2 \pi \omega(N))}^{A_{v, w} / 2} \int_{1 /(2 \pi \omega(N))}^{A_{u, w} / 2} \frac{1}{z_{1}+1} \frac{1}{z_{2}+1} \frac{1}{\left(C_{w, v} z_{1}+1\right)^{1 / 2}} \frac{1}{\left(C_{w, u} z_{2}+1\right)^{1 / 2}} d z_{1} d z_{2} \\
= & {\left[\int_{0}^{\infty} \frac{1}{z_{1}+1} \frac{1}{\left(C_{w, v} z_{1}+1\right)^{1 / 2}} d z_{1}\right]\left[\int_{0}^{\infty} \frac{1}{z_{2}+1} \frac{1}{\left(C_{w, u} z_{2}+1\right)^{1 / 2}} d z_{2}\right] . }
\end{aligned}
$$


Let us consider the first of these two integrals. We further bound it as follows:

$$
\begin{aligned}
& \int_{0}^{\infty} \frac{1}{z_{1}+1} \frac{1}{\left(C_{w, v} z_{1}+1\right)^{1 / 2}} d z_{1} \\
& \quad \leq\left\{\begin{array}{ll}
\int_{0}^{\infty} \frac{1}{\left(z_{1}+1\right)^{3 / 2}} d z_{1}, & \text { if } C_{w, v} \geq 1 \\
\int_{0}^{\infty} \frac{1}{z_{1}+1} \frac{1}{\left(C_{w, v} z_{1}+C_{w, v}\right)^{1 / 2}} d z_{1}=\frac{1}{C_{w, v}^{1 / 2}} \int_{0}^{\infty} \frac{1}{\left(z_{1}+1\right)^{3 / 2}} d z_{1}, & \text { if } C_{w, v}<1
\end{array},\right.
\end{aligned}
$$

The second integral in (7.7) is bounded analogously. Now, we split $D_{t}$ into four sub domains:

1. $t_{u}, t_{v} \leq t_{w}$ (that is, $C_{w, v}, C_{w, u} \geq 1$ );

2. $t_{u} \leq t_{w}$ but $t_{v}>t_{w}$ (that is, $C_{w, u} \geq 1$ and $C_{w, v}<1$ );

3. $t_{v} \leq t_{w}$ but $t_{u}>t_{w}$ (that is, $C_{w, v} \geq 1$ and $C_{w, u}<1$ );

4. $t_{u}, t_{v}>t_{w}$ (that is, $C_{w, v}, C_{w, u}<1$ ).

We denote by $D_{i}$ the domain considered in Case $i$, for $i=1, \ldots, 4$. Hence, we have

$$
\left(\begin{array}{l}
N \\
3
\end{array}\right) \mathbb{E}(T(u, v ; w) \vartheta(u, v ; w))=\left(\begin{array}{c}
N \\
3
\end{array}\right) \sum_{i=1}^{4} \mathbb{E}\left(T(u, v ; w) \vartheta(u, v ; w) \mathbf{1}_{\left\{\left(t_{u}, t_{v}, t_{w}\right) \in D_{i}\right\}}\right) .
$$

\section{Setting}

$$
\begin{aligned}
I^{\prime}(u, v, w):= & \left(\frac{v}{N}\right)^{2} \frac{e^{2 \zeta t_{w}}}{C_{w, v} C_{w, u}} \max \left\{\frac{1}{C_{w, v}^{1 / 2}}, 1\right\} \max \left\{\frac{1}{C_{w, u}^{1 / 2}}, 1\right\} \\
& \times\left[\int_{0}^{\infty} \frac{1}{\left(z_{1}+1\right)^{3 / 2}} d z_{1}\right]^{2},
\end{aligned}
$$

then (7.7) and (7.8) imply that

$$
I(u, v, w) \leq I^{\prime}(u, v, w) .
$$

We now consider each of the four summands in (7.9) separately.

Case 1. In this sub domain, we have

$$
\frac{e^{2 \zeta t_{w}}}{C_{w, v} C_{w, u}}=e^{\zeta t_{w}+\frac{\zeta}{2}\left(t_{v}+t_{u}\right)}
$$


We substitute this into the expression for $I^{\prime}$ and we integrate over $D_{1}$, using Claim 3.4, thus obtaining

$$
\begin{gathered}
\left(\begin{array}{c}
N \\
3
\end{array}\right) \mathbb{E}\left(T(u, v ; w) \mathbf{1}_{\left(t_{w}, t_{u}, t_{v}\right) \in D_{1}}\right) \leq N^{3} \int_{D_{1}} I^{\prime}(u, v, w) d t_{w} d t_{v} d t_{u} \\
\asymp N \int_{0}^{R / 2-\omega(N)} \int_{0}^{t_{w}} \int_{0}^{t_{w}} e^{(\zeta-\alpha) t_{w}+(\zeta / 2-\alpha) t_{v}+(\zeta / 2-\alpha) t_{u}} d t_{u} d t_{v} d t_{w} \\
\asymp N \int_{0}^{R / 2-\omega(N)} e^{(\zeta-\alpha) t_{w}}\left[\int_{0}^{t_{w}} e^{(\zeta / 2-\alpha) t_{u}} d t_{u}\right]^{2} d t_{w} \\
\lesssim N \int_{0}^{R / 2-\omega(N)} e^{(\zeta-\alpha) t_{w}} d t_{w} \lesssim \begin{cases}N, & \text { if } \zeta / \alpha<1 \\
R N, & \text { if } \zeta / \alpha=1 \\
N^{2-\alpha / \zeta}, & \text { if } \zeta / \alpha>1\end{cases}
\end{gathered}
$$

Cases 2 and 3. Here, it suffices to consider only Case 2, where $t_{u} \leq t_{w}$ and $t_{v}>t_{w}$. Case 3 is treated in exactly the same way and gives the same outcome. Here, $C_{w, u} \geq 1$ but $C_{w, v}<1$. Hence, by (7.8), the factor that appears in $I^{\prime}$ becomes

$$
\frac{e^{2 \zeta t_{w}}}{C_{w, v}^{3 / 2} C_{w, u}}<\frac{e^{2 \zeta t_{w}}}{C_{w, v}^{2} C_{w, u}}=e^{\frac{\zeta}{2} t_{w}+\zeta t_{v}+\frac{\zeta}{2} t_{u}} .
$$

Thus, using again Claim 3.4, we have

$$
\begin{aligned}
& \left(\begin{array}{l}
N \\
3
\end{array}\right) \mathbb{E}\left(T(u, v ; w) \mathbf{1}_{\left(t_{w}, t_{u}, t_{v}\right) \in D_{2}}\right) \leq N^{3} \int_{D_{2}} I^{\prime}(u, v, w) d t_{w} d t_{v} d t_{u} \\
& \asymp N \int_{0}^{R / 2-\omega(N)} \int_{t_{w}}^{R / 2-\omega(N)} \int_{0}^{t_{w}} e^{(\zeta / 2-\alpha) t_{w}+(\zeta-\alpha) t_{v}+(\zeta / 2-\alpha) t_{u}} d t_{u} d t_{v} d t_{w} \\
& \lesssim N \int_{0}^{R / 2} \int_{0}^{R / 2} \int_{0}^{R / 2} e^{(\zeta / 2-\alpha) t_{w}+(\zeta-\alpha) t_{v}+(\zeta / 2-\alpha) t_{u}} d t_{u} d t_{v} d t_{w} \\
& =N\left[\int_{0}^{R / 2} e^{(\zeta-\alpha) t_{v}} d t_{v}\right]\left[\int_{0}^{R / 2} e^{(\zeta / 2-\alpha) t_{u}} d t_{u}\right]^{2} \lesssim \begin{cases}N, & \text { if } \zeta / \alpha<1 \\
R N, & \text { if } \zeta / \alpha=1 . \\
N^{2-\alpha / \zeta,}, & \text { if } \zeta / \alpha>1\end{cases}
\end{aligned}
$$

Case 4. Now, the factor that appears in $I^{\prime}$ becomes

$$
\frac{e^{2 \zeta t_{w}}}{C_{w, v}^{3 / 2} C_{w, u}^{3 / 2}}=e^{\frac{\zeta}{2} t_{w}+\frac{3}{2} \frac{\zeta}{2}\left(t_{v}+t_{u}\right)}
$$


Hence,

$$
\begin{aligned}
& \left(\begin{array}{l}
N \\
3
\end{array}\right) \mathbb{E}\left(T(u, v ; w) \mathbf{1}_{\left(t_{w}, t_{u}, t_{v}\right) \in D_{4}}\right) \leq N^{3} \int_{D_{4}} I^{\prime}(u, v, w) d t_{w} d t_{v} d t_{u} \\
& \asymp N \int_{0}^{R / 2-\omega(N)} \int_{t_{w}}^{R / 2-\omega(N)} \int_{t_{w}}^{R / 2-\omega(N)} e^{\left(\frac{3}{2} \frac{\zeta}{2}-\alpha\right)\left(t_{u}+t_{v}\right)+(\zeta / 2-\alpha) t_{w}} d t_{u} d t_{v} d t_{w} \\
& \leq N\left[\int_{0}^{R / 2-\omega(N)} e^{(\zeta / 2-\alpha) t_{w}} d t_{w}\right]\left[\int_{0}^{R / 2} e^{(3 \zeta / 4-\alpha) t_{u}} d t_{u}\right]^{2} \\
& \lesssim \begin{cases}N, & \text { if } \zeta / \alpha<4 / 3 \\
R^{2} N, & \text { if } \zeta / \alpha=4 / 3 . \\
N^{1+3 / 2-2 \alpha / \zeta}, & \text { if } \zeta / \alpha>4 / 3\end{cases}
\end{aligned}
$$

The last exponent is equal to $2.5-2 \alpha / \zeta<2-\alpha / \zeta$, as $1 / 2<\alpha / \zeta$. Therefore, by plugging all these estimates into (7.9), we finally obtain

$$
\left(\begin{array}{l}
N \\
3
\end{array}\right) \mathbb{E}(T(u, v ; w) \vartheta(u, v ; w))=o(\mathbb{E}(\widehat{\Lambda}))
$$

\subsection{Proof of Proposition 2.2(ii) $(\beta<1)$}

To prove Proposition 2.2(ii) for $\beta<1$, it also suffices to show (7.1). Recall (6.9)

$$
\begin{aligned}
& \mathbb{E}\left(T(u, v ; w) \vartheta(u, v ; w) \mid t_{u}, t_{v}, t_{w}\right) \\
& \quad \lesssim \frac{1}{A_{u, w} A_{v, w}} \int_{\frac{\omega(N)}{2 \pi}}^{A_{v, w} / 2} \int_{\frac{\omega(N)}{2 \pi}}^{A_{u, w} / 2} \frac{1}{z_{1}^{\beta}+1} \frac{1}{z_{2}^{\beta}+1} \frac{1}{\left(C_{w, v} z_{1}+C_{w, u} z_{2}\right)^{\beta}+1} d z_{1} d z_{2} .
\end{aligned}
$$

The condition $z_{2} \geq \omega(N) / 2 \pi$ implies that

$$
\frac{1}{z_{2}^{\beta}+1} \lesssim\left(\frac{1}{\omega(N)}\right)^{\beta} .
$$

Setting $y=C_{w, v} z_{1}+C_{w, u} z_{2}$, we have

$$
d z_{1} d z_{2}=\frac{d z_{1} d y}{C_{w, u}}
$$

with

$$
y \in\left(C_{w, v} z_{1}+C_{w, u} \frac{\omega(N)}{2 \pi}, C_{w, v} z_{1}+C_{w, u} A_{v, w} / 2\right) .
$$


So, we obtain:

$$
\begin{aligned}
& \mathbb{E}\left(T(u, v ; w) \vartheta(u, v ; w) \mid t_{u}, t_{v}, t_{w}\right) \\
& \quad \lesssim\left(\frac{1}{\omega(N)}\right)^{\beta} \frac{1}{A_{u, w} A_{v, w} C_{w, u}} \int_{\frac{\omega(N)}{2 \pi}}^{A_{u, w} / 2} \frac{1}{z_{1}^{\beta}} \int_{C_{w, v} z_{1}+C_{w, u} \frac{\omega(N)}{2 \pi}}^{C_{w, v} z_{1}+C_{w, u} A_{v, w} / 2} \frac{1}{y^{\beta}} d y d z_{1} .
\end{aligned}
$$

We extend the inner integration interval using an upper bound on $z_{1}$. In particular, because $z_{1} \leq A_{u, w} / 2$, we have $C_{w, v} z_{1}+C_{w, u} A_{v, w} / 2 \leq\left(C_{w, v} A_{u, w}+C_{w, u} A_{v, w}\right) / 2$. Note that

$$
\begin{aligned}
& C_{w, v} A_{u, w}=\exp \left(\frac{\zeta}{2}\left(t_{w}-t_{v}+R-t_{u}-t_{w}\right)\right)=A_{u, v}, \\
& C_{w, u} A_{v, w}=A_{u, v} .
\end{aligned}
$$

Hence,

$$
C_{w, v} z_{1}+C_{w, u} A_{v, w} / 2 \leq A_{u, v} .
$$

Also, because $z_{1} \geq \frac{\omega(N)}{2 \pi}$, we have $C_{w, v} z_{1}+C_{w, u} \frac{\omega(N)}{2 \pi} \geq\left(C_{w, v}+C_{w, u}\right) \frac{\omega(N)}{2 \pi}$. Then, we will obtain an upper bound by extending the integration interval to

$$
y \in\left(\left(C_{w, v}+C_{w, u}\right) \frac{\omega(N)}{2 \pi}, A_{u, v}\right) .
$$

Hence, we obtain

$$
\begin{aligned}
& \mathbb{E}\left(T(u, v ; w) \vartheta(u, v ; w) \mid t_{u}, t_{v}, t_{w}\right) \\
& \quad \lesssim\left(\frac{1}{\omega(N)}\right)^{\beta} \frac{1}{A_{u, w} A_{v, w} C_{w, u}} \int_{\frac{\omega(N)}{2 \pi}}^{A_{u, w} / 2} \frac{1}{z_{1}^{\beta}} d z_{1} \int_{\left(C_{w, v}+C_{w, u} \frac{\omega(N)}{2 \pi}\right.}^{A_{u, v}} \frac{1}{y^{\beta}} d y \\
& \quad \stackrel{(7.13)}{\lesssim}\left(\frac{1}{\omega(N)}\right)^{\beta} \frac{1}{A_{u, w} A_{u, v}} A_{u, w}^{1-\beta} A_{u, v}^{1-\beta} \asymp\left(\frac{1}{\omega(N)}\right)^{\beta} \frac{1}{\left(A_{u, v} A_{u, w}\right)^{\beta}} .
\end{aligned}
$$

Now, we integrate over $\left(t_{u}, t_{v}, t_{w}\right) \in D_{t}=[0, R / 2-\omega(N)]^{3}$ using Claim 3.4, and obtain

$$
\begin{aligned}
& \mathbb{E}(T(u, v ; w) \vartheta(u, v ; w)) \\
& \quad \lesssim \int_{0}^{R / 2-\omega(N)} \int_{0}^{R / 2-\omega(N)} \int_{0}^{R / 2-\omega(N)}\left(\frac{1}{\omega(N)}\right)^{\beta} \frac{e^{-\alpha\left(t_{u}+t_{v}+t_{w}\right)}}{\left(A_{u, v} A_{u, w}\right)^{\beta}} d t_{u} d t_{v} d t_{w} .
\end{aligned}
$$

Elementary integration now yields:

$$
\mathbb{E}(T(u, v ; w) \vartheta(u, v ; w)) \lesssim \begin{cases}(\omega(N))^{-\beta} N^{-2 \beta} & \text { if } \beta \zeta / \alpha<1 \\ (\omega(N))^{-\beta} R N^{-2 \beta} & \text { if } \beta \zeta / \alpha=1 \\ (\omega(N))^{-\beta} N^{-\beta-\alpha / \zeta} e^{-(\beta \zeta-\alpha) \omega(N)} & \text { if } \beta \zeta / \alpha>1\end{cases}
$$

Multiplying this by $\left(\begin{array}{c}N \\ 3\end{array}\right)$ and comparing the outcome with (2.3), we now deduce Proposition 2.2(ii) for $\beta<1$ from (6.2). 


\section{PROOF OF PROPOSITION 2.4}

In this section, we bound from above the expected number of atypical triangles, that is, those triangles that contain at least one vertex of type greater than $R / 2-\omega(N)$. We will do this only for the case $\zeta / \alpha>1$, because for the case $\zeta / \alpha<1$ the argument is straightforward.

\subsection{Case $\zeta / \alpha<1$}

Note that if $\widetilde{\Lambda} \geq 1$, then there is a vertex of type at least $R / 2-\omega(N)$. But by Corollary 3.5 , a.a.s. all vertices have type at most

$$
\frac{\zeta}{2 \alpha} R+\omega(N)<\frac{R}{2}-\omega(N)
$$

for $N$ sufficiently large. Hence, in this case, $3 \widetilde{T} \leq \widetilde{\Lambda}=0$, a.a.s.

\subsection{Case $\zeta / \alpha \geq 1$}

As a preliminary observation, we point out that the probability that three vertices form an atypical triangle is bounded from above by the probability that they form an incomplete triangle.

For every triple $u, v, w$ of distinct vertices, we let $\mathbf{1}_{\Delta_{a}(u, v, w)}$ denote the indicator random variable that is equal to 1 if and only if the vertices $u, v$, and $w$ form a triangle and at least one of these vertices is atypical. Similarly, we let $\mathbf{1}_{\Lambda_{a}(u, v ; w)}$ be the indicator random variable that is equal to 1 if and only if the vertices $u, v$, and $w$ form an incomplete triangle with $w$ as the pivoting vertex and at least one of these vertices is atypical.

We split $\mathbb{E}\left(\mathbf{1}_{\Delta_{a}(u, v, w)}\right)$ as follows:

$$
\begin{aligned}
\mathbb{E}\left(\mathbf{1}_{\Delta_{a}(u, v, w)}\right)= & 3 \mathbb{E}\left(\mathbf{1}_{\Delta_{a}(u, v, w)} \mathbf{1}_{t_{u}, t_{v} \leq R / 2-\omega(N) ; t_{w}>R / 2-\omega(N)}\right) \\
& +\mathbb{E}\left(\mathbf{1}_{\Delta_{a}(u, v, w)} \mathbf{1}_{t_{u}, t_{v}, t_{w}>R / 2-\omega(N)}\right) \\
& +3 \mathbb{E}\left(\mathbf{1}_{\Delta_{a}(u, v, w)} \mathbf{1}_{t_{u} \leq R / 2-\omega(N) ; t_{v}, t_{w}>R / 2-\omega(N)}\right) .
\end{aligned}
$$

To deduce Proposition 2.4, it suffices to show that

$$
\left.\begin{array}{l}
N^{3} \mathbb{E}\left(\mathbf{1}_{\Delta_{a}(u, v, w)} \mathbf{1}_{t_{u}, t_{v} \leq R / 2-\omega(N) ; t_{w}>R / 2-\omega(N)}\right) \\
N^{3} \mathbb{E}\left(\mathbf{1}_{\Delta_{a}(u, v, w)} \mathbf{1}_{t_{u}, t_{v}, t_{w}>R / 2-\omega(N)}\right) \\
N^{3} \mathbb{E}\left(\mathbf{1}_{\Delta_{a}(u, v, w)} \mathbf{1}_{t_{u}, t_{v} \leq R / 2-\omega(N) ; t_{w}>R / 2-\omega(N)}\right)
\end{array}\right\}=o(\mathbb{E}(\hat{\Lambda}))
$$

Hence, the second part of Proposition 2.4 will follow from Markov's inequality.

We will estimate each of these terms separately. We will be using the following general inequality:

$$
\mathbb{E}\left(\mathbf{1}_{\Delta_{a}(u, v, w)} \mathbf{1}_{E}\right) \leq \mathbb{E}\left(\mathbf{1}_{\Lambda_{a}(u, v ; w)} \mathbf{1}_{E}\right),
$$

where $E$ denotes any event. The event $E$ will be specified according to the case we consider.

Now recall the definitions of $\beta^{\prime}$ and $\delta$ :

$$
\beta^{\prime}:=\left\{\begin{array}{ll}
\beta, & \text { if } \beta<1 \\
1, & \text { if } \beta \geq 1
\end{array} \quad \text { and } \quad \delta:=\left\{\begin{array}{ll}
0, & \text { if } \beta \neq 1 \\
1, & \text { if } \beta=1
\end{array} .\right.\right.
$$


For sake of clarity, we will defer many of the technical calculations to Appendix A.

\subsection{Case $t_{u}, t_{v}, t_{w}>R / 2-\omega(N)$}

In this case, we bound $\mathbb{E}\left(\mathbf{1}_{\Delta_{a}(u, v ; w)}\right)$ by the probability that $u, v, w$ have type greater than $R / 2-\omega(N)$ :

$$
\mathbb{E}\left(\mathbf{1}_{\Delta_{a}(u, v ; w)} \mathbf{1}_{t_{u}, t_{v}, t_{w}>R / 2-\omega(N)}\right) \lesssim \int_{R / 2-\omega(N)}^{R} \int_{R / 2-\omega(N)}^{R} \int_{R / 2-\omega(N)}^{R} e^{-\alpha\left(t_{u}+t_{v}+t_{w}\right)} d t_{u} d t_{v} d t_{w} .
$$

Now, it is easy to check that

$$
t_{u}+t_{v}+t_{w}>\frac{3}{2} R-3 \omega(N)
$$

From these observations, we can deduce the following:

$$
\begin{aligned}
& \mathbb{E}\left(\mathbf{1}_{\Delta_{a}(u, v, w)} \mathbf{1}_{t_{u}, t_{v}, t_{w}>R / 2-\omega(N)}\right) \\
& \quad \lesssim \int_{R / 2-\omega(N)}^{R} \int_{R / 2-\omega(N)}^{R} \int_{R / 2-\omega(N)}^{R} e^{-\alpha\left(\frac{3}{2} R-3 \omega(N)\right)} d t_{u} d t_{v} d t_{w} \\
& \quad \asymp R^{3} e^{-\frac{3 \alpha}{\zeta} \frac{\zeta R}{2}} e^{3 \alpha \omega(N)} \asymp R^{3} N^{-\frac{3 \alpha}{\zeta}} e^{3 \alpha \omega(N)} .
\end{aligned}
$$

It suffices to show that if $\omega(N)$ is sufficiently slow growing, then we have

$$
R^{3} N^{3-\frac{3 \alpha}{\zeta}} e^{3 \alpha \omega(N)} \ll \mathbb{E}(\widehat{\Lambda}) .
$$

Indeed, this follows from the following conditions, which are easy to verify (cf. Proposition 2.1).

(i) for $\beta \geq 1$ (i.e., $\beta^{\prime}=1$ ), we have

$$
\begin{cases}3-3 \alpha / \zeta<1, & \text { if } \zeta / \alpha=1 \\ 3-3 \alpha / \zeta<2-\alpha / \zeta, & \text { if } \zeta / \alpha>1\end{cases}
$$

because the latter is equivalent to $2 \alpha / \zeta>1$.

(ii) for $\beta<1$ (which, by the definition of the model, implies $\beta \zeta / \alpha<2$ ), we have

$$
\left\{\begin{array}{ll}
3-3 \alpha / \zeta<3-2 \beta, & \text { if } \beta \zeta / \alpha \leq 1 \\
3-3 \alpha / \zeta<3-\beta-\alpha / \zeta, & \text { if } \beta \zeta / \alpha>1
\end{array},\right.
$$

because the former is equivalent to $\beta \zeta / \alpha<3 / 2$ and the latter is equivalent to $\alpha / \beta \zeta>$ $1 / 2$.

\subsection{Case $t_{u} \leq R / 2-\omega(N)$ and $t_{v}, t_{w}>R / 2-\omega(N)$}

Here, we will make use of (8.3) and bound the probability that three vertices form a triangle by the probability that they form an incomplete triangle. In particular, we shall be considering the case when the incomplete triangle is pivoted at $w$. Under this assumption, we consider the following two sub cases: 
1. $t_{u}+t_{w} \leq R-\omega(N)$ with $t_{v}+t_{w}>R-2 \omega(N)$;

2. $t_{u}+t_{w}>R-\omega(N)$ with $t_{v}+t_{w}>R-2 \omega(N)$.

Assume without loss of generality that $t_{v}>t_{w}$. The domain of integration of sub case 1 becomes

$$
D_{1}:=\left\{0<t_{u} \leq R-t_{w}-\omega(N), t_{w}<t_{v}<R, R / 2-\omega(N)<t_{w}<R\right\} .
$$

Note first that for any $u, v \in \mathrm{V}_{N}$, we have $\ln \left(A_{u, v}\right) \lesssim \ln (N) \asymp R$. Thus, applying Lemma 3.1, we obtain

$$
\begin{aligned}
& \mathbb{E}\left(\mathbf{1}_{\Lambda_{a}(u, v ; w)} \mathbf{1}_{t_{u} \leq R / 2-\omega(N) ; t_{v}, t_{w}>R / 2-\omega(N)} \mathbf{1}_{\left(t_{u}, t_{v}, t_{w}\right) \in D_{1}}\right) \\
& \quad \lesssim \int_{R / 2-\omega(N)}^{R} \int_{t_{w}}^{R} \int_{0}^{R-t_{w}-\omega(N)}\left(\frac{R^{\delta}}{A_{u, w}}\right)^{\beta^{\prime}} e^{-\alpha\left(t_{u}+t_{v}+t_{w}\right)} d t_{u} d t_{v} d t_{w}=: \phi_{1} .
\end{aligned}
$$

Multiplying by $N^{3}$, the estimates in (A.1) imply (8.2). In fact, it is easy to show that the following conditions hold.

(i) For $\beta \geq 1$, we have

$$
\left\{\begin{array}{ll}
5 / 2-2 \alpha / \zeta<1, & \text { if } \zeta / \alpha=1 \\
5 / 2-2 \alpha / \zeta<2-\alpha / \zeta, & \text { if } \zeta / \alpha>1
\end{array},\right.
$$

where the latter is equivalent to $\alpha / \zeta>1 / 2$ (that is, $\zeta / \alpha<2$ ).

(ii) For $\beta<1$, we have

$$
\left\{\begin{array}{ll}
3-\beta / 2-2 \alpha / \zeta<3-2 \beta, & \text { if } \beta \zeta / \alpha \leq 1 \\
3-\beta / 2-2 \alpha / \zeta<3-\beta-\alpha / \zeta, & \text { if } \beta \zeta / \alpha>1
\end{array},\right.
$$

where the former is equivalent to $\beta \zeta / \alpha<4 / 3$ and the latter is equivalent to $\alpha / \beta \zeta>1 / 2$ (that is, $\beta \zeta / \alpha<2$ ).

In sub case 2, the domain of integration is

$$
D_{2}:=\left\{R-t_{w}-\omega(N)<t_{u}<R / 2-\omega(N), t_{w}<t_{v}<R, R / 2-\omega(N)<t_{w}<R\right\} .
$$

Hence, we get

$$
\begin{aligned}
& \mathbb{E}\left(\mathbf{1}_{\Lambda_{a}(u, v ; w)} \mathbf{1}_{t_{u} \leq R / 2-\omega(N) ; t_{v}, t_{w}>R / 2-\omega(N)} \mathbf{1}_{\left(t_{u}, t_{v}, t_{w}\right) \in D_{2}}\right) \\
& \quad \lesssim \int_{R / 2-\omega(N)}^{R} \int_{t_{w}}^{R} \int_{R-t_{w}-\omega(N)}^{R / 2} e^{-\alpha\left(t_{u}+t_{v}+t_{w}\right)} d t_{u} d t_{v} d t_{w}=: \phi_{2} .
\end{aligned}
$$

This is estimated in (A.2). Multiplying by $N^{3}$, we obtain (8.2). Indeed, the following inequalities are easy to verify.

(i) For $\beta \geq 1$, we have

$$
\begin{cases}3-3 \alpha / \zeta<1, & \text { if } \zeta / \alpha=1 \\ 3-3 \alpha / \zeta<2-\alpha / \zeta, & \text { if } \zeta / \alpha>1\end{cases}
$$


where the last inequality holds because $\alpha / \zeta>1 / 2$ (that is, $\zeta / \alpha<2$ ).

(ii) For $\beta<1$, we have

$$
\left\{\begin{array}{ll}
3-3 \alpha / \zeta<3-2 \beta, & \text { if } \beta \zeta / \alpha \leq 1 \\
3-3 \alpha / \zeta<3-\beta-\alpha / \zeta, & \text { if } \beta \zeta / \alpha>1
\end{array},\right.
$$

where the former holds because $\beta \zeta / \alpha \leq 1<3 / 2$ and the latter because $\alpha / \zeta>1 / 2$ and $\beta<1$.

\subsection{Case $t_{u}, t_{v} \leq R / 2-\omega(N)$ and $t_{w}>R / 2-\omega(N)$}

Under our current assumptions, we have four possible sub cases:

1. $t_{u}+t_{w} \leq R-2 \omega(N)$ with $t_{v}+t_{w} \leq R-2 \omega(N)$;

2. $t_{u}+t_{w} \leq R-2 \omega(N)$ with $t_{v}+t_{w}>R-2 \omega(N)$;

3. $t_{u}+t_{w}>R-2 \omega(N)$ with $t_{v}+t_{w} \leq R-2 \omega(N)$;

4. $t_{u}+t_{w}>R-2 \omega(N)$ with $t_{v}+t_{w}>R-2 \omega(N)$.

We denote the $i$ th domain by $D_{i}$. We need to treat each situation separately, starting with sub case 1. We shall use Lemma 3.1:

$$
\begin{aligned}
& \mathbb{E}\left(\mathbf{1}_{\Delta_{a}(u, v, w)} \mathbf{1}_{t_{u}, t_{v} \leq R / 2-\omega(N) ; t_{w}>R / 2-\omega(N)} \mathbf{1}_{\left(t_{u}, t_{v}, t_{w}\right) \in D_{1}}\right) \\
& \quad \leq \mathbb{E}\left(\mathbf{1}_{\Lambda_{a}(v, w ; u)} \mathbf{1}_{t_{u}, t_{v} \leq R / 2-\omega(N) ; t_{w}>R / 2-\omega(N)} \mathbf{1}_{\left(t_{u}, t_{v}, t_{w}\right) \in D_{1}}\right) \\
& \quad \lesssim \int_{R / 2-\omega(N)}^{R-2 \omega(N)} \int_{0}^{R-t_{w}-2 \omega(N)} \int_{0}^{R-t_{w}-2 \omega(N)}\left(\frac{R^{2 \delta}}{A_{u, v} A_{u, w}}\right)^{\beta^{\prime}} \\
& \times e^{-\alpha\left(t_{u}+t_{v}+t_{w}\right)} d t_{u} d t_{v} d t_{w}=: \phi_{3} .
\end{aligned}
$$

The asymptotic growth of $\phi_{3}$ is determined by the ratio $\beta^{\prime} \zeta / \alpha$, as in (A.3) in Appendix A. To deduce (8.2) on this sub domain, we multiply (A.3) by $N^{3}$ and compare the resulting exponents of $N$ with those in Proposition 2.1.

(i) For $\beta \geq 1$ (that is, $\beta^{\prime}=1$ ) we have

$$
\left\{\begin{array}{ll}
3-\frac{3}{2} \beta^{\prime}-\alpha / \zeta<1, & \text { if } \zeta / \alpha=1 \\
3-\beta^{\prime} / 2-2 \alpha / \zeta<2-\alpha / \zeta, & \text { if } \zeta / \alpha>1
\end{array},\right.
$$

where the latter holds because $\alpha / \zeta>1 / 2$ (that is, $\zeta / \alpha<2$ ).

(ii) For $\beta<1$ (where $\beta^{\prime}=\beta$ ) we have

$$
\begin{cases}3-\frac{3}{2} \beta-\alpha / \zeta<3-2 \beta, & \text { if } \beta \zeta / \alpha<1 \\ 3-\frac{5}{2} \beta<3-2 \beta, & \text { if } \beta \zeta / \alpha=1 \\ 3-\frac{\beta}{2}-2 \alpha / \zeta<3-\beta-\alpha / \zeta, & \text { if } \beta \zeta / \alpha>1\end{cases}
$$

because $\alpha / \zeta>1 / 2$ and $\beta<1$. 
With regard to Case 2 (as well as Case 3) we have the following:

$$
\begin{aligned}
& \mathbb{E}\left(\mathbf{1}_{\Delta_{a}(u, v, w)} \mathbf{1}_{t_{u}, t_{v} \leq R / 2-\omega(N) ; t_{w}>R / 2-\omega(N)} \mathbf{1}_{\left(t_{u}, t_{v}, t_{w}\right) \in D_{2}}\right) \\
& \quad \leq \mathbb{E}\left(\mathbf{1}_{\Lambda_{a}(v, w ; u)} \mathbf{1}_{t_{u}, t_{v} \leq R / 2-\omega(N) ; t_{w}>R / 2-\omega(N)} \mathbf{1}_{\left(t_{u}, t_{v}, t_{w}\right) \in D_{2}}\right) \\
& \quad \lesssim \int_{R / 2-\omega(N)}^{R} \int_{0}^{R-t_{w}-2 \omega(N)} \int_{R-t_{w}-2 \omega(N)}^{R / 2-\omega(N)}\left(\frac{R^{2 \delta}}{A_{u, v} A_{u, w}}\right)^{\beta^{\prime}} \\
& \quad \times e^{-\alpha\left(t_{u}+t_{v}+t_{w}\right)} d t_{v} d t_{u} d t_{w}=: \phi_{4} .
\end{aligned}
$$

As in the previous case, this expression depends on the ratio $\beta^{\prime} \zeta / \alpha$. The statement follows multiplying (A.4) by $N^{3}$ and comparing the exponents of $N$ with those in Proposition 2.1.

(i) For $\beta \geq 1$, we get

$$
\begin{cases}2-2 \alpha / \zeta<1, & \text { if } \zeta / \alpha=1 \\ 3-3 \alpha / \zeta<2-\alpha / \zeta, & \text { if } 1<\zeta / \alpha<2\end{cases}
$$

(ii) For $\beta<1$, we get

$$
\begin{cases}3-\beta-2 \alpha / \zeta<3-2 \beta, & \text { if } \beta \zeta / \alpha \leq 1 \\ 3-3 \alpha / \zeta<3-\beta-\alpha / \zeta, & \text { if } \beta \zeta / \alpha>1\end{cases}
$$

Case 4 is treated in a similar way:

$$
\begin{aligned}
\mathbb{E} & \left(\mathbf{1}_{\Delta_{a}(u, v, w)} \mathbf{1}_{t_{u}, t_{v} \leq R / 2-\omega(N) ; t_{w}>R / 2-\omega(N)} \mathbf{1}_{\left(t_{u}, t_{v}, t_{w}\right) \in D_{4}}\right) \\
\lesssim & \int_{R / 2-\omega(N)}^{R} \int_{R-t_{w}-2 \omega(N)}^{R / 2-\omega(N)} \int_{R-t_{w}-2 \omega(N)}^{R / 2-\omega(N)}\left(\frac{R^{\delta}}{A_{u, v}}\right)^{\beta^{\prime}} \\
& \times e^{-\alpha\left(t_{u}+t_{v}+t_{w}\right)} d t_{u} d t_{v} d t_{w}=: \phi_{5} .
\end{aligned}
$$

We estimate this in (A.5) in Appendix A. As previously, the statement follows from the multiplication of (A.5) by $N^{3}$.

(i) For $\beta \geq 1$ we get

$$
\begin{cases}2-2 \alpha / \zeta<1, & \text { if } \zeta / \alpha=1 \\ 3-3 \alpha / \zeta<2-\alpha / \zeta, & \text { if } 1<\zeta / \alpha<2\end{cases}
$$

(ii) For $\beta<1$ we get

$$
\begin{cases}3-\beta-2 \alpha / \zeta<3-2 \beta, & \text { if } \beta \zeta / \alpha \leq 1 \\ 3-3 \alpha / \zeta<3-\beta-\alpha / \zeta, & \text { if } \beta \zeta / \alpha>1\end{cases}
$$




\section{CONCLUSIONS}

In this article we give a precise characterization of the presence of clustering in random geometric graphs on the hyperbolic plane in terms of its parameters. We focus on the range of parameters where these random graphs have a linear number of edges and their degree distribution follows a power law. We quantify the existence of clustering, furthermore, in the part of the random graph that consists of vertices that have type at most $t$, where $0<t<R$, we show that the clustering coefficient there is bounded away from 0 . More importantly, we determine exactly how this quantity depends on the parameters of the random graph.

The present work is a step towards establishing such random graphs as a suitable model for complex networks. Together with [17] and [19], our results show that for certain values of the parameters, such random graphs do capture two of the fundamental properties of complex networks: power-law degree distribution as well as clustering.

A natural next step in this direction is the study of the typical distances (in terms of hops) between vertices. More precisely, it would be interesting to investigate the distance between two typical vertices, and how the values of the parameters influence this quantity. In other words, for which values of $\beta$ and $\zeta / \alpha$ is the resulting random graph what is commonly called a small world?

\section{APPENDICES}

\section{A. Auxiliary Calculations}

In this section, we show the technical calculations needed to finish the proofs in Section 8. Recall that

$$
\beta^{\prime}=\left\{\begin{array}{ll}
\beta & \text { if } \beta<1 \\
1 & \text { if } \beta \geq 1
\end{array} \quad \text { and } \quad \delta=\left\{\begin{array}{ll}
0 & \text { if } \beta \neq 1 \\
1 & \text { if } \beta=1
\end{array} .\right.\right.
$$

In the proof of Proposition 2.4, we defined the functions $\phi_{1}, \ldots, \phi_{5}$, which we calculate explicitly in this section.

We start with

$$
\phi_{1} \leq R^{\delta} \int_{R / 2-\omega(N)}^{R} \int_{t_{w}}^{R} \int_{0}^{R-t_{w}-\omega(N)}\left(\frac{1}{A_{u, w}}\right)^{\beta^{\prime}} e^{-\alpha\left(t_{u}+t_{v}+t_{w}\right)} d t_{u} d t_{v} d t_{w} .
$$

Then, we have

$$
\begin{aligned}
\phi_{1} & \lesssim \frac{R^{\delta}}{N^{\beta^{\prime}}} \int_{R / 2-\omega(N)}^{R} \int_{t_{w}}^{R} \int_{0}^{R-t_{w}-\omega(N)} e^{\left(\beta^{\prime} \zeta / 2-\alpha\right) t_{u}+\left(\beta^{\prime} \zeta / 2\right) t_{w}-\alpha\left(t_{v}+t_{w}\right)} d t_{u} d t_{v} d t_{w} \\
& \lesssim \frac{R^{\delta}}{N^{\beta^{\prime}}} \int_{R / 2-\omega(N)}^{R} \int_{t_{w}}^{R} e^{\left(\beta^{\prime} \zeta / 2\right) t_{w}} e^{-\alpha\left(t_{v}+t_{w}\right)} d t_{v} d t_{w} \\
& \lesssim \frac{R^{\delta}}{N^{\beta^{\prime}}} \int_{R / 2-\omega(N)}^{R} e^{\left(\beta^{\prime} \zeta / 2-2 \alpha\right) t_{w}} d t_{w} \lesssim \frac{R^{\delta}}{N^{\beta^{\prime}}} e^{\left(\beta^{\prime} \zeta / 2-2 \alpha\right)(R / 2-\omega(N))} .
\end{aligned}
$$

Hence, we have

$$
\phi_{1} \lesssim R^{\delta} N^{-\beta^{\prime} / 2-2 \alpha / \zeta} e^{-\left(\beta^{\prime} \zeta / 2-2 \alpha\right) \omega(N)}
$$


We now consider

$$
\phi_{2} \leq \int_{R / 2-\omega(N)}^{R} \int_{t_{w}}^{R} \int_{R-t_{w}-\omega(N)}^{R / 2} e^{-\alpha\left(t_{u}+t_{v}+t_{w}\right)} d t_{u} d t_{v} d t_{w} .
$$

A calculation similar to the previous case yields

$$
\begin{aligned}
\phi_{2} & \lesssim \int_{R / 2-\omega(N)}^{R} \int_{t_{w}}^{R} e^{-\alpha\left(R-t_{w}-\omega(N)\right)} e^{-\alpha\left(t_{v}+t_{w}\right)} d t_{v} d t_{w} \\
& =e^{-\alpha(R-\omega(N))} \int_{R / 2-\omega(N)}^{R} \int_{t_{w}}^{R} e^{-\alpha t_{v}} d t_{v} d t_{w} \\
& \lesssim N^{-2 \alpha / \zeta} e^{\alpha \omega(N)} \int_{R / 2-\omega(N)}^{R} e^{-\alpha t_{w}} d t_{w} \lesssim N^{-2 \alpha / \zeta} e^{\alpha \omega(N)} e^{-\alpha(R / 2-\omega(N))}
\end{aligned}
$$

And, finally, we obtain

$$
\phi_{2} \lesssim N^{-3 \alpha / \zeta} e^{2 \alpha \omega(N)}
$$

Now, we consider

$$
\begin{aligned}
\phi_{3}= & \int_{R / 2-\omega(N)}^{R-2 \omega(N)} \int_{0}^{R-t_{w}-2 \omega(N)} \int_{0}^{R-t_{w}-2 \omega(N)}\left(\frac{R^{2 \delta}}{A_{u, v} A_{u, w}}\right)^{\beta^{\prime}} \\
& \times e^{-\alpha\left(t_{u}+t_{v}+t_{w}\right)} d t_{u} d t_{v} d t_{w} \\
\leq & \frac{R^{2 \delta}}{N^{2 \beta^{\prime}}} \int_{R / 2-\omega(N)}^{R-2 \omega(N)} \int_{0}^{R-t_{w}-2 \omega(N)} \int_{0}^{R-t_{w}-2 \omega(N)} e^{\left(\frac{\beta^{\prime} \zeta}{2}-\alpha\right)\left(t_{w}+t_{v}\right)+\left(\beta^{\prime} \zeta-\alpha\right) t_{u}} \\
& \times d t_{u} d t_{v} d t_{w} \\
\lesssim & \frac{R^{2 \delta}}{N^{2 \beta^{\prime}}} \int_{R / 2-\omega(N)}^{R-2 \omega(N)} e^{\left(\frac{\beta^{\prime} \zeta}{2}-\alpha\right) t_{w}}\left[\int_{0}^{R-t_{w}-2 \omega(N)} e^{\left(\beta^{\prime} \zeta-\alpha\right) t_{u}} d t_{u}\right] d t_{w} .
\end{aligned}
$$

Now, the order of magnitude of this integral depends on the ratio $\beta^{\prime} \zeta / \alpha$.

For $\beta^{\prime} \zeta / \alpha \leq 1$, we have

$$
\begin{aligned}
\phi_{3} & \lesssim \frac{R^{2 \delta+1}}{N^{2 \beta^{\prime}}} \int_{R / 2-\omega(N)}^{R-2 \omega(N)} e^{\left(\frac{\beta^{\prime} \zeta}{2}-\alpha\right) t_{w}} d t_{w} \lesssim \frac{R^{2 \delta+1}}{N^{2 \beta^{\prime}}} e^{\left(\frac{\beta^{\prime} \zeta}{2}-\alpha\right) R / 2} e^{-\left(\beta^{\prime} \zeta / 2-\alpha\right) \omega(N)} \\
\asymp & \frac{R^{2 \delta+1} N^{\beta^{\prime} / 2-\alpha / \zeta}}{N^{2 \beta^{\prime}}} e^{-\left(\beta^{\prime} \zeta / 2-\alpha\right) \omega(N)}=R^{2 \delta+1} N^{-\frac{3}{2} \beta^{\prime}-\alpha / \zeta} e^{-\left(\beta^{\prime} \zeta / 2-\alpha\right) \omega(N)} .
\end{aligned}
$$


Finally, when $\beta^{\prime} \zeta / \alpha>1$, we have

$$
\begin{aligned}
\phi_{3} & \lesssim \frac{R^{2 \delta} e^{\left(\beta^{\prime} \zeta-\alpha\right) R}}{N^{2 \beta^{\prime}}} e^{-2\left(\beta^{\prime} \zeta-\alpha\right) \omega(N)} \int_{R / 2-\omega(N)}^{R-2 \omega(N)} e^{\left(\frac{\beta^{\prime} \zeta}{2}-\alpha\right) t_{w}-\left(\beta^{\prime} \zeta-\alpha\right) t_{w}} d t_{w} \\
& \asymp \frac{R^{2 \delta} N^{2\left(\beta^{\prime}-\alpha / \zeta\right)}}{N^{2 \beta^{\prime}}} e^{-2\left(\beta^{\prime} \zeta-\alpha\right) \omega(N)} \int_{R / 2-\omega(N)}^{R-2 \omega(N)} e^{-\beta^{\prime} \zeta t_{w} / 2} d t_{w} \\
& \asymp \frac{R^{2 \delta} N^{2\left(\beta^{\prime}-\alpha / \zeta\right)} e^{-\frac{\beta^{\prime} \zeta}{2} R / 2}}{N^{2 \beta^{\prime}}} e^{-\left(3 \beta^{\prime} \zeta / 2-2 \alpha\right) \omega(N)} \\
& \asymp \frac{R^{2 \delta} N^{2\left(\beta^{\prime}-\alpha / \zeta\right)-\frac{\beta^{\prime}}{2}}}{N^{2 \beta^{\prime}}} e^{-\left(3 \beta^{\prime} \zeta / 2-2 \alpha\right) \omega(N)}=R^{2 \delta} N^{-\frac{\beta^{\prime}}{2}-2 \alpha / \zeta} e^{-\left(3 \beta^{\prime} \zeta / 2-2 \alpha\right) \omega(N)} .
\end{aligned}
$$

Therefore,

$$
\phi_{3} \lesssim\left\{\begin{array}{ll}
R^{2 \delta+1} N^{-\frac{3}{2} \beta^{\prime}-\alpha / \zeta} e^{-\left(\beta^{\prime} \zeta / 2-\alpha\right) \omega(N)}, & \text { if } \beta^{\prime} \zeta / \alpha \leq 1 \\
R^{2 \delta} N^{-\frac{\beta^{\prime}}{2}-2 \alpha / \zeta} e^{-\left(3 \beta^{\prime} \zeta / 2-2 \alpha\right) \omega(N)}, & \text { if } \beta^{\prime} \zeta / \alpha>1
\end{array} .\right.
$$

Now, we consider the function

$$
\begin{aligned}
\phi_{4} \leq & R^{2 \delta} \int_{R / 2-\omega(N)}^{R} \int_{0}^{R-t_{w}-2 \omega(N)} \int_{R-t_{w}-2 \omega(N)}^{R / 2-\omega(N)}\left(\frac{1}{A_{u, v} A_{u, w}}\right)^{\beta^{\prime}} \\
& \times e^{-\alpha\left(t_{u}+t_{v}+t_{w}\right)} d t_{v} d t_{u} d t_{w} \\
\asymp & \frac{R^{2 \delta}}{N^{2 \beta^{\prime}}} \int_{R / 2-\omega(N)}^{R} \int_{0}^{R-t_{w}-2 \omega(N)} \int_{R-t_{w}-2 \omega(N)}^{R / 2-\omega(N)} e^{\left(\beta^{\prime} \zeta-\alpha\right) t_{u}} \\
& \times e^{\left(\frac{\beta^{\prime} \zeta}{2}-\alpha\right)\left(t_{v}+t_{w}\right)} d t_{v} d t_{u} d t_{w} .
\end{aligned}
$$

Therefore, integrating with respect to $t_{v}$ and recalling that $\beta^{\prime} \zeta / 2<\alpha$, we obtain:

$$
\begin{aligned}
\phi_{4} \lesssim & \frac{R^{2 \delta} e^{\left(\frac{\beta^{\prime} \zeta}{2}-\alpha\right) R}}{N^{2 \beta^{\prime}}} e^{-\left(\beta^{\prime} \zeta-2 \alpha\right) \omega(N)} \\
& \times \int_{R / 2-\omega(N)}^{R} \int_{0}^{R-t_{w}-2 \omega(N)} e^{\left(\beta^{\prime} \zeta-\alpha\right) t_{u}-\left(\frac{\beta^{\prime} \zeta}{2}-\alpha\right) t_{w}+\left(\frac{\beta^{\prime} \zeta}{2}-\alpha\right) t_{w}} d t_{u} d t_{w} \\
\asymp & \frac{R^{2 \delta} N^{\beta^{\prime}-2 \alpha / \zeta}}{N^{2 \beta^{\prime}}} e^{-\left(\beta^{\prime} \zeta-2 \alpha\right) \omega(N)} \int_{R / 2-\omega(N)}^{R} \int_{0}^{R-t_{w}-2 \omega(N)} e^{\left(\beta^{\prime} \zeta-\alpha\right) t_{u}} d t_{u} d t_{w} .
\end{aligned}
$$

Now, the behavior of the latter integral depends on the value of $\beta^{\prime} \zeta / \alpha$. If $\beta^{\prime} \zeta / \alpha \leq 1$, then

$$
\phi_{4} \lesssim R^{2 \delta+2} N^{-\beta^{\prime}-2 \alpha / \zeta} e^{-\left(\beta^{\prime} \zeta-2 \alpha\right) \omega(N)}
$$


However, for $\beta^{\prime} \zeta / \alpha>1$, we have

$$
\begin{aligned}
\phi_{4} & \lesssim R^{2 \delta} N^{-\beta^{\prime}-2 \alpha / \zeta} e^{-\left(\beta^{\prime} \zeta-2 \alpha\right) \omega(N)} \int_{R / 2-\omega(N)}^{R} e^{\left(\beta^{\prime} \zeta-\alpha\right)\left(R-t_{w}-2 \omega(N)\right)} d t_{w} \\
& =R^{2 \delta} N^{-\beta^{\prime}-2 \alpha / \zeta} e^{\left(\beta^{\prime} \zeta-\alpha\right) R} e^{-\left(3 \beta^{\prime} \zeta-4 \alpha\right) \omega(N)} \int_{R / 2-\omega(N)}^{R} e^{-\left(\beta^{\prime} \zeta-\alpha\right) t_{w}} d t_{w} \\
& \lesssim R^{2 \delta} N^{-\beta^{\prime}-2 \alpha / \zeta} e^{\left(\beta^{\prime} \zeta-\alpha\right) R-\left(\beta^{\prime} \zeta-\alpha\right) R / 2} e^{-\left(2 \beta^{\prime} \zeta-3 \alpha\right) \omega(N)} \\
& \asymp R^{2 \delta} N^{-\beta^{\prime}-2 \alpha / \zeta} e^{\left(\beta^{\prime} \zeta-\alpha\right) R / 2} e^{-\left(2 \beta^{\prime} \zeta-3 \alpha\right) \omega(N)} \\
& \asymp R^{2 \delta} N^{-\beta^{\prime}-2 \alpha / \zeta+\beta^{\prime}-\alpha / \zeta} e^{-\left(2 \beta^{\prime} \zeta-3 \alpha\right) \omega(N)}=R^{2 \delta} N^{-3 \alpha / \zeta} e^{-\left(2 \beta^{\prime} \zeta-3 \alpha\right) \omega(N)} .
\end{aligned}
$$

Therefore,

$$
\phi_{4} \lesssim \begin{cases}R^{2 \delta+2} N^{-\beta^{\prime}-2 \alpha / \zeta} e^{-\left(\beta^{\prime} \zeta-2 \alpha\right) \omega(N)}, & \text { if } \beta^{\prime} \zeta / \alpha \leq 1 \\ R^{2 \delta} N^{-3 \alpha / \zeta} e^{-\left(3 \beta^{\prime} \zeta-4 \alpha\right) \omega(N)}, & \text { if } \beta^{\prime} \zeta / \alpha>1\end{cases}
$$

Finally, we consider

$$
\phi_{5}=\int_{R / 2-\omega(N)}^{R} \int_{R-t_{w}-2 \omega(N)}^{R / 2-\omega(N)} \int_{R-t_{w}-2 \omega(N)}^{R / 2-\omega(N)}\left(\frac{R^{\delta}}{A_{u, v}}\right)^{\beta^{\prime}} e^{-\alpha\left(t_{u}+t_{v}+t_{w}\right)} d t_{u} d t_{v} d t_{w} .
$$

The integral of this expression is estimated as follows:

$$
\begin{aligned}
& \int_{R / 2-\omega(N)}^{R} \int_{R-t_{w}-2 \omega(N)}^{R / 2-\omega(N)} \int_{R-t_{w}-2 \omega(N)}^{R / 2-\omega(N)}\left(\frac{R^{\delta}}{A_{u, v}}\right)^{\beta^{\prime}} e^{-\alpha\left(t_{u}+t_{v}+t_{w}\right)} d t_{u} d t_{v} d t_{w} \\
& \asymp \frac{R^{\delta}}{N^{\beta^{\prime}}} \int_{R / 2-\omega(N)}^{R} \int_{R-t_{w}-2 \omega(N)}^{R / 2-\omega(N)} \int_{R-t_{w}-2 \omega(N)}^{R / 2-\omega(N)} e^{\frac{\beta^{\prime} \zeta}{2}\left(t_{u}+t_{v}\right)-\alpha\left(t_{u}+t_{v}+t_{w}\right)} d t_{u} d t_{v} d t_{w} \\
& \lesssim \frac{R^{\delta}}{N^{\beta^{\prime}}} \int_{R / 2-\omega(N)}^{R} e^{-\alpha t_{w}+2\left(\beta^{\prime} \zeta / 2-\alpha\right)\left(R-t_{w}-2 \omega(N)\right)} d t_{w} \\
& =\frac{R^{\delta} e^{2\left(\beta^{\prime} \zeta / 2-\alpha\right)(R-2 \omega(N))}}{N^{\beta^{\prime}}} \int_{R / 2-\omega(N)}^{R} e^{-\left(\beta^{\prime} \zeta-\alpha\right) t_{w}} d t_{w} \\
& =R^{\delta} \frac{N^{2 \beta^{\prime}-4 \alpha / \zeta}}{N^{\beta^{\prime}}} e^{-4\left(\beta^{\prime} \zeta / 2-\alpha\right) \omega(N)} \int_{R / 2-\omega(N)}^{R} e^{-\left(\beta^{\prime} \zeta-\alpha\right) t_{w}} d t_{w} .
\end{aligned}
$$

Hence, there are three cases according to the value of $\beta^{\prime} \zeta / \alpha$, thus obtaining

$$
\phi_{5} \lesssim\left\{\begin{array}{cc}
R^{\delta} N^{-\beta^{\prime}-2 \alpha / \zeta} e^{-4\left(\beta^{\prime} \zeta / 2-\alpha\right) \omega(N)}, & \text { if } \beta^{\prime} \zeta / \alpha<1 \\
R^{\delta+1} N^{\beta^{\prime}-4 \alpha / \zeta} e^{-4\left(\beta^{\prime} \zeta-2 \alpha\right) \omega(N)} & \\
=R^{\delta+1} N^{-3 \alpha / \zeta} e^{-4\left(\beta^{\prime} \zeta-2 \alpha\right) \omega(N)}, & \text { if } \beta^{\prime} \zeta / \alpha=1 \\
R^{\delta} N^{-3 \alpha / \zeta} e^{-\left(\beta^{\prime} \zeta-3 \alpha\right) \omega(N)}, & \text { if } \beta^{\prime} \zeta / \alpha>1
\end{array}\right.
$$




\section{B. Proof of Lemma 1.1}

We begin with the hyperbolic law of cosines:

$$
\begin{aligned}
\cosh (\zeta d(u, v))= & \cosh \left(\zeta\left(R-t_{u}\right)\right) \cosh \left(\zeta\left(R-t_{v}\right)\right) \\
& -\sinh \left(\zeta\left(R-t_{u}\right)\right) \sinh \left(\zeta\left(R-t_{v}\right)\right) \cos \left(\theta_{u, v}\right) .
\end{aligned}
$$

The right-hand side of the above becomes:

$$
\begin{aligned}
\cosh \left(\zeta\left(R-t_{u}\right)\right) \cosh \left(\zeta\left(R-t_{v}\right)\right)-\sinh \left(\zeta\left(R-t_{u}\right)\right) \sinh \left(\zeta\left(R-t_{v}\right)\right) \cos \left(\theta_{u, v}\right) \\
=\frac{e^{\zeta\left(2 R-\left(t_{u}+t_{v}\right)\right)}}{4}\left(\left(1+e^{-2 \zeta\left(R-t_{u}\right)}\right)\left(1+e^{-2 \zeta\left(R-t_{v}\right)}\right)\right. \\
\left.\quad-\left(1-e^{-2 \zeta\left(R-t_{u}\right)}\right)\left(1-e^{-2 \zeta\left(R-t_{v}\right)}\right) \cos \left(\theta_{u, v}\right)\right) \\
=\frac{e^{\zeta\left(2 R-\left(t_{u}+t_{v}\right)\right)}}{4}\left(1-\cos \left(\theta_{u, v}\right)+\left(1+\cos \left(\theta_{u, v}\right)\right)\left(e^{-2 \zeta\left(R-t_{u}\right)}+e^{-2 \zeta\left(R-t_{v}\right)}\right)\right. \\
\left.\quad+O\left(e^{-2 \zeta\left(2 R-\left(t_{u}+t_{v}\right)\right)}\right)\right)
\end{aligned}
$$

Therefore,

$$
\begin{aligned}
\cosh (\zeta d(u, v)) \leq & \frac{e^{\zeta\left(2 R-\left(t_{u}+t_{v}\right)\right)}}{4}\left(1-\cos \left(\theta_{u, v}\right)+2\left(e^{-2 \zeta\left(R-t_{u}\right)}+e^{-2 \zeta\left(R-t_{v}\right)}\right)\right. \\
& \left.+O\left(e^{-2 \zeta\left(2 R-\left(t_{u}+t_{v}\right)\right)}\right)\right)
\end{aligned}
$$

Because $t_{u}+t_{v}<R-c_{0}$, the last error term is $O\left(N^{-4}\right)$. Also, it is a basic trigonometric identity that $1-\cos \left(\theta_{u, v}\right)=2 \sin ^{2}\left(\frac{\theta_{u, v}}{2}\right)$. The latter is at most $\frac{\theta_{u, v}^{2}}{2}$. Therefore, the upper bound on $\theta_{u, v}$ yields:

$$
\begin{aligned}
\cosh (\zeta d(u, v)) \leq & \frac{e^{\zeta\left(2 R-\left(t_{u}+t_{v}\right)\right)}}{4}\left(\frac{\theta_{u, v}^{2}}{2}+2\left(e^{-2 \zeta\left(R-t_{u}\right)}+e^{-2 \zeta\left(R-t_{v}\right)}\right)+O\left(\frac{1}{N^{4}}\right)\right) \\
\leq & \frac{e^{\zeta\left(2 R-\left(t_{u}+t_{v}\right)\right)}}{4}\left(2(1-\varepsilon)^{2} e^{\zeta\left(t_{u}+t_{v}-(1-\delta) R\right)}+2\left(e^{-2 \zeta\left(R-t_{u}\right)}+e^{-2 \zeta\left(R-t_{v}\right)}\right)\right) \\
& +O(1) \\
= & (1-\varepsilon)^{2} \frac{e^{\zeta(1+\delta) R}}{2}+\frac{1}{2}\left(e^{\zeta\left(t_{u}-t_{v}\right)}+e^{\zeta\left(t_{v}-t_{u}\right)}\right)+O(1) \\
< & (1-\varepsilon)^{2} \frac{e^{\zeta(1+\delta) R}}{2}+\varepsilon \frac{e^{\zeta(1+\delta) R}}{2}+O(1)<\frac{e^{\zeta(1+\delta) R}}{2},
\end{aligned}
$$

for $N$ sufficiently large and $c_{0}$ such that $e^{-c_{0}}<\frac{1}{2} \varepsilon$, because $t_{u}+t_{v}<(1-|\delta|) R-c_{0}$ and $t_{u}, t_{v} \geq 0$. This implies that $t_{u}-t_{v}, t_{v}-t_{u}<(1+\delta) R-c_{0}$ and, therefore, $\frac{1}{2}\left(e^{\zeta\left(t_{u}-t_{v}\right)}+e^{\zeta\left(t_{v}-t_{u}\right)}\right)<\frac{1}{2}\left(e^{\zeta(1+\delta) R-c_{0}}+e^{\zeta(1+\delta) R-c_{0}}\right)<\varepsilon \frac{e^{\zeta(1+\delta) R}}{2}$. Also, because $\cosh (\zeta d(u, v))>\frac{1}{2} e^{\zeta d(u, v)}$, it follows that $d(u, v)<(1+\delta) R$. 
To deduce the second part of the lemma, we consider a lower bound on (B.1), using the lower bound on $\theta_{u, v}$ :

$$
\begin{aligned}
\cosh (\zeta d(u, v)) & \geq \frac{e^{\zeta\left(2 R-\left(t_{u}+t_{v}\right)\right)}}{4}\left(1-\cos \left(\theta_{u, v}\right)\right)+O(1) \\
& \geq \frac{e^{\zeta\left(2 R-\left(t_{u}+t_{v}\right)\right)}}{4}\left(1-\cos \left(2(1+\varepsilon) e^{\frac{\zeta}{2}\left(t_{u}+t_{v}-(1-\delta) R\right)}\right)\right)+O(1)
\end{aligned}
$$

Using again that $1-\cos (\theta)=2 \sin ^{2}\left(\frac{\theta}{2}\right)$, we deduce that

$$
1-\cos \left(2(1+\varepsilon) e^{\frac{\zeta}{2}\left(t_{u}+t_{v}-(1-\delta) R\right)}\right)=2 \sin ^{2}\left(\frac{1}{2} 4(1+\varepsilon)^{2} e^{\zeta\left(t_{u}+t_{v}-(1-\delta) R\right)}\right) .
$$

Because $t_{u}+t_{v}<(1-|\delta|) R-c_{0}$, it follows that $t_{u}+t_{v}-(1-\delta) R<-c_{0}$. So the latter is

$$
\sin \left(\frac{1}{2} 4(1+\varepsilon)^{2} e^{\zeta\left(t_{u}+t_{v}-(1-\delta) R\right)}\right)>2\left(1+\frac{\varepsilon}{2}\right)^{2} e^{\zeta\left(t_{u}+t_{v}-(1-\delta) R\right)},
$$

for $N$ and $c_{0}$ large enough, using the Taylor's expansion of the sine function around 0 . Substituting this bound into (B.2), we have

$$
\cosh (\zeta d(u, v)) \geq\left(1+\frac{\varepsilon}{2}\right)^{2} \frac{e^{\zeta(1+\delta) R}}{2}+O(1)
$$

Thus, if $d(u, v) \leq(1+\delta) R$, the left-hand side would be smaller than the right-hand side, which would lead to a contradiction.

\section{FUNDING} 293619.

Research supported by a Marie Curie Career Integration Grant PCIG09-GA2011-

\section{REFERENCES}

[1] R. Albert and A.-L. Barabási. "Statistical Mechanics of Complex Networks." Reviews of Modern Physics 74 (2002), 47-97.

[2] J. Balogh, B. Bollobás, M. Krivelevich, T. Müller and M. Walters. "Hamilton Cycles in Random Geometric Graphs.” Ann. Appl. Probab. 21:3 (2011), 1053-1072.

[3] A.-L. Barabási and R. Albert. "Emergence of Scaling in Random Networks." Science 286 (1999), 509-512.

[4] A. Barrat and M. Weigt. "On the Properties of Small-World Network Models." European Physical Journal B 13:3 (2000), 547-560.

[5] M. Bloznelis. "Degree and Clustering Coefficient in Sparse Random Intersection Graphs." Ann. Appl. Probab. 23:3 (2013), 1254-1289.

[6] B. Bollobás. Random Graphs. Cambridge, UK: Cambridge University Press, 2001.

[7] B. Bollobás and O. Riordan. "Mathematical Results on Scale-Free Random Graphs." In Handbook of Graphs and Networks: From the Genome to the Internet, edited by S. Bornholdt and H. G. Schuster, pp. 1-34. Berlin: Wiley-VCH, 2003.

[8] B. Bollobás, S. Janson, and O. Riordan. "Sparse Random Graphs with Clustering." Random Structures Algorithms 38 (2011), 269-323. 
[9] K. Börner, J. T. Maru, and R. L. Goldstone. "Colloquium Paper: Mapping Knowledge Domains: The Simultaneous Evolution of Author and Paper Networks." Proc. Natl. Acad. Sci. USA 101 (2004), 5266-5273.

[10] F. Chung and L. Lu. "The Average Distances in Random Graphs with Given Expected Degrees." Proc. Natl. Acad. Sci. USA 99 (2002), 15879-15882.

[11] F. Chung and L. Lu. "Connected Components in Random Graphs with Given Expected Degree Sequences." Annals of Combinatorics 6 (2002), 125-145.

[12] F. Chung and L. Lu. Complex Graphs and Networks. Providence, RI: AMS, 2006.

[13] E. Coupechoux and M. Lelarge. "How Clustering Affects Epidemics in Random Networks." In Proceedings of the 5th International Conference on Network Games, Control and Optimization, (NetGCooP 2011), pp. 1-7. Paris: IEEE, 2011.

[14] S. N. Dorogovtsev. Lectures on Complex Networks. Oxford, UK: Oxford University Press, 2010.

[15] N. Eggemann and S. D. Noble. "The Clustering Coefficient of a Scale-Free Random Graph." Discrete Applied Mathematics 159:10 (2011), 953-965.

[16] I. Foudalis, K. Jain, C. H. Papadimitriou, and M. Sideri. "Modeling Social Networks Through User Background and Behavior." In Proceedings of the 8th International Workshop on Algorithms and Models for the Web Graph (WAW 2011), edited by A. Frieze, P. Horn, and P. Prałat, Lecture Notes on Computer Science 6732, pp. 85-102. Berlin, Heidelberg: Springer, 2011.

[17] N. Fountoulakis. "On a Geometrization of the Chung-Lu Model for Complex networks." Journal of Complex Networks, 3:3 (2015), 361-387.

[18] E. N. Gilbert. "Random Plane Networks." J. Soc. Indust. Appl. Math. 9 (1961), 533-543.

[19] L. Gugelmann, K. Panagiotou, and U. Peter. "Random Hyperbolic Graphs: Degree Sequence and Clustering." In Proceedings of the 39th International Colloquium on Automata, Languages and Programming, edited by A. Czumaj et al., Lecture Notes in Computer Science 7392, pp. 573-585. Berlin, Heidelberg: Springer, 2012.

[20] R. Hafner. "The Asymptotic Distribution of Random Clumps.” Computing 10 (1972), 335-351.

[21] S. Janson, T. Łuczak, and A. Ruciński. Random Graphs. Hoboken, NJ: Wiley-Interscience, 2001.

[22] D. Krioukov, F. Papadopoulos, M. Kitsak, A. Vahdat, and M. Boguñá. "Hyperbolic Geometry of Complex Networks." Phys. Rev. E 82 (2010), 036106.

[23] R. D. Luce and A. D. Perry. "A Method of Matrix Analysis of Group Structure." Psychometrika 14:1 (1949), 95-116.

[24] C. McDiarmid and T. Müller. "On the Chromatic Number of Random Geometric Graphs." Combinatorica 31:4 (2011), 423-488.

[25] R. Meester and R. Roy. Continuum Percolation. Cambridge, UK: Cambridge University Press, 1996.

[26] F. Menczer. "Growing and Navigating the Small World Web by Local Content." Proc. Natl. Acad. Sci. USA 99 (2002), 14014-14019.

[27] P. Mörters and E. Jacob. "Spatial Preferential Attachment Networks: Power Laws and Clustering Coefficients." Ann. Appl. Probab. 25 (2015), 632-662.

[28] M. E. Newman, S. H. Strogatz, and D. J. Watts. "Random Graphs with Arbitrary Degree Distributions and Their Applications." Phys. Rev. E 64 (2001), 026118.

[29] M. Penrose. Random Geometric Graphs. Oxford, UK: Oxford University Press, 2003.

[30] D. J. Watts and S. H. Strogatz. "Collective Dynamics of "Small-World" Networks." Nature 393 (1998), 440-442. 